\title{
Overview article
}

\section{(CPHY-19-0027.R1)}

\section{Mitochondria in the Pulmonary Vasculature in Health and Disease: Oxygen-sensing,}

\section{Metabolism, and Dynamics}

Asish Dasgupta ${ }^{1}$, Danchen Wu ${ }^{1}$, Lian Tian ${ }^{1}$, Ping Y. Xiong ${ }^{1}$, Kimberly J. Dunham-Snary ${ }^{1}$, Kuang-

Hueih Chen ${ }^{1}$, Elahe Alizadeh ${ }^{2}$, Mehras Motamed ${ }^{1}$, François Potus ${ }^{1}$, Charles C.T. Hindmarch ${ }^{2}$ and Stephen L. Archer ${ }^{1}$

${ }^{1}$ Department of Medicine, Queen’s University, 94 Stuart St., Kingston, Ontario, K7L 3N6

Canada

${ }^{2}$ Queen's Cardiopulmonary Unit (QCPU), Translational Institute of Medicine (TIME), Department of Medicine, Queen’s University, 94 Stuart St., Kingston, Ontario, K7L 3N6, Canada

Corresponding author

\section{Stephen L. Archer MD. FRCP(C), FAHA, FACC}

\section{Tier 1 CRC Mitochondrial Dynamics}

Professor and Head, Department of Medicine, Queen's University

Program Medical Director Kingston Health Sciences Centre and St. Mary's of the Lake Hospital Etherington Hall, Room 3041

94 Stuart St., Kingston, Ontario, Canada, K7L 3N6

Preferred E-mail: stephen.archer@queensu.ca

Telephone: 613 533-6327

Fax: 613 533-6695 


\section{Abstract (word count 247 words, maximum words: 250)}

In lung vascular cells mitochondria serve a canonical metabolic role, governing energy homeostasis. In addition, mitochondria exist in dynamic networks which serve noncanonical functions, including regulation of redox signaling, cell cycle, apoptosis and mitochondrial quality control. Mitochondria in pulmonary artery smooth muscle cells (PASMC) are oxygen sensors and initiate hypoxic pulmonary vasoconstriction. Acquired dysfunction of mitochondrial metabolism and dynamics contribute to a cancer-like phenotype in pulmonary arterial hypertension (PAH). Acquired mitochondrial abnormalities, such as increased pyruvate dehydrogenase kinase (PDK) and pyruvate kinase muscle isoform 2 (PKM2) expression, which increase uncoupled glycolysis (the Warburg phenomenon), are implicated in PAH. Warburg metabolism sustains energy homeostasis by the inhibition of oxidative metabolism which reduces mitochondrial apoptosis, allowing unchecked cell accumulation. Warburg metabolism is initiated by induction of a pseudohypoxic state, in which DNA methyltransferase (DNMT)-mediated changes in redox signalling cause normoxic activation of HIF-1 $\alpha$ and increase PDK expression. Furthermore, mitochondrial division is coordinated with nuclear division through a process called mitotic fission. Increased mitotic fission in PAH, driven by increased fission and reduced fusion favours rapid cell cycle progression and apoptosis resistance. Downregulation of the mitochondrial calcium uniporter complex (MCUC) occurs in PAH and is one potential unifying mechanism linking Warburg metabolism and mitochondrial fission. Mitochondrial metabolic and dynamic disorders combine to promote the hyperproliferative, apoptosis-resistant, phenotype in PAH PASMC, endothelial cells and fibroblasts. Understanding the molecular mechanism regulating mitochondrial metabolism and dynamics has permitted indentification of new biomarkers, nuclear and CT imaging modalities, and new therapeutic targets for PAH. 
Key Words: Oxygen sensing, hypoxic pulmonary vasoconstriction, reactive oxygen species (ROS), Dynamin related protein 1 (Drp1), mitofusin-2, HIF-1 $\alpha$, mitochondrial calcium uniporter (MCU), MiD49 and MiD51, pyruvate kinase, pyruvate dehydrogenase kinase (PDK), Peroxisome proliferator-activated receptor gamma (PPAR $\gamma$ ),pyruvate dehydrogenase (PDH), micro RNA (miR)-138-3p, Clinical trials, ${ }^{18}$ fluordeoxyglucose positron emission tomography $\left({ }^{18}\right.$ FDG PET), trimetazidine, ranolazine, dichloroacetate, right ventricle 


\section{Didactic Synopsis (150 maximum 150 words).}

1. Mitochondria in resistance pulmonary artery smooth muscle cells (PASMC) are oxygen sensors that transduce alveolar $\mathrm{O}_{2}$ to a diffusible redox signal that regulates ion channels and enzymes, leading to hypoxic pulmonary vasoconstriction.

2. Acquired abnormalities of mitochondrial metabolism and dynamics promotes a cancer like hyperproliferative, apoptosis-resistant, phenotype in all PAH vascular cells .

3. In PAH, acquired metabolic abnormalities include an increase in uncoupled aerobic glycolysis (Warburg metabolism) similar to that seen in cancer.

4. In PAH, Warburg metabolism is partially mediated by upregulation of PDK and an increased PKM2/PKM1 ratio.

5. Mitochondrial fragmentation, a hallmark of PAH, reflects an increased fission/fusion ratio caused by activation of Drp1, upregulation of MiD49 and MiD51, and downregulation of Mfn2.

6. Newly recognized mitochondrial pathways offer potential therapeutic targets for pulmonary vascular diseases, including: PDK inhibitors, PKM2 inhibitors, miR mimics and anti-miRs, inhibitors of Drp1 and its binding partners, and Mfn2 augmentation. 
Table 1: Abbreviations

\begin{tabular}{|c|c|}
\hline$\left[{ }^{18}\right.$ F]FTHA & $14(\mathrm{R}, \mathrm{S})-\left[{ }^{18} \mathrm{~F}\right]$ fluoro-6-thia-heptadecanoic acid \\
\hline 3'-UTR & 3'-untranslated region \\
\hline 4-AP & 4-aminopyridine \\
\hline 6MWT & 6-minute walk test \\
\hline$\alpha-\mathbf{K G}$ & $\alpha$-ketoglutarate \\
\hline$\beta$-AR & $\beta$-adrenergic receptor \\
\hline$\Delta \psi_{m}$ & membrane potential \\
\hline AMPK & AMP-activated protein kinase \\
\hline ATP & adenosine triphosphate \\
\hline BAD & Bcl-2 associated agonist of cell death \\
\hline BET & bromodomain and extra-terminal domain \\
\hline BMIPP & 15-(p-iodophenyl)-3-(R,S)-methylpentadecanoic acid \\
\hline BMPR2 & bone morphogenetic protein receptor type 2 \\
\hline BOEC & blood outgrowth endothelial cells \\
\hline BPD & bronchopulmonary dysplasia \\
\hline CAMKII & $\mathrm{Ca}^{2+} /$ calmodulin-dependent kinase II \\
\hline CDK4 & cyclin-dependent kinase 4 \\
\hline $\mathrm{CoCl2}$ & cobalt chloride \\
\hline Cpc-PH & pre-capillary pulmonary hypertension \\
\hline CPT-1 & carnitine palmitoyltransferase 1 \\
\hline CtBP1 & C-terminal binding protein \\
\hline CTEPH & chronic thromboembolic pulmonary hypertension \\
\hline CXCL12 & C-X-C motif chemokine 12 \\
\hline $\mathbf{D A}$ & ductus arteriosus \\
\hline DCA & dichloroacetate \\
\hline DNMT & DNA methyltransferases \\
\hline DON & 6-diazo-5-oxo-L-norleucine \\
\hline Drp1 & dynamin related protein 1 \\
\hline Dyn2 & dynamin 2 \\
\hline ECHO & echocardiography \\
\hline ECM & extracellular matrix \\
\hline EMRE & essential MCU regulator \\
\hline eNOS & endothelial nitric oxide synthase \\
\hline ER & endoplasmic reticulum \\
\hline ERK & extracellular-signal-regulated kinase \\
\hline ET & endothelin \\
\hline ETC & electron transport chain \\
\hline
\end{tabular}




\begin{tabular}{|l|l|}
\hline EZH2 & enhancer of zeste homologue 2 \\
\hline FADH2 & flavin adenine dinucleotide \\
\hline FAO & fatty acid oxidation \\
\hline FAS & fatty acid synthase \\
\hline FDG & 2-[ ${ }^{18}$ F-Fluoro-2-deoxy-D-glucose \\
\hline FGF2 & fibroblast growth factor 2 \\
\hline FHR & fawn hooded rats \\
\hline FIH-1 & factor inhibiting HIF-1 $\alpha$ \\
\hline Fis1 & fission protein 1 \\
\hline G6PD & glucose-6-phosphate dehydrogenase \\
\hline GEO & Gene Expression Omnibus \\
\hline GLS1 & glutaminase \\
\hline Glut1 & glucose transporter 1 \\
\hline GRIM-19 & mortality-19 protein \\
\hline GSK-3 $\beta$ & glycogen synthase kinase 3 beta \\
\hline GTP & guanosine triphosphate \\
\hline H2O 2 & hydrogen peroxide \\
\hline HAT & histone acetyl transferase \\
\hline HDAC & histone deacetylase \\
\hline HFpEF & heart failure with preserved ejection fraction \\
\hline HFrEF & heart failure with reduced ejection fraction \\
\hline HIF-1 $\boldsymbol{\alpha}$ & hypoxia inducible factor-1 $\boldsymbol{\alpha}$ \\
\hline HK2 & hexokinase II \\
\hline HMOX1 & heme Oxygenase 1 \\
\hline HOSS & homeostatic Oxygen Sensing System \\
\hline HPH & hypoxia-induced pulmonary hypertension \\
\hline HPV & hypoxic pulmonary vasoconstriction \\
\hline HR2 & heptad repeat \\
\hline HRE & hypoxia response element \\
\hline Ik & potassium current \\
\hline IL1 $\beta$ & interleukin 1 $\boldsymbol{\beta}$ \\
\hline IMM & inner mitochondrial membrane \\
\hline IRE1 $\boldsymbol{\alpha}$ & inositol-requiring protein 1 $\alpha$ \\
\hline JNK & Jun N-terminal kinase \\
\hline K2P & two-pore ${ }^{+}$channels \\
\hline KCNA5 & Kv1.5 \\
\hline KLF5 & Krüppel-like Factor 5 \\
\hline KO & knockout \\
\hline L-NAME & No-nitro-L-arginine methyl ester \\
\hline
\end{tabular}




\begin{tabular}{|l|l|}
\hline LC3 & light chain \\
\hline LDH & lactate dehydrogenase \\
\hline MAM & mitochondria associated membrane \\
\hline MAP & mitogen-activated protein \\
\hline MARCH5 & membrane-associated ring finger (C3HC4) 5 \\
\hline MBF & myocardial blood flow \\
\hline MCD & malonyl-CoA decarboxylase \\
\hline MCT & monocrotaline \\
\hline MCUC & mitochondrial calcium uniporter complex \\
\hline MetS & metabolic syndrome \\
\hline MFF & mitochondrial fission factor \\
\hline mfn & mitofusin \\
\hline MGU & myocardial glucose uptake \\
\hline MIBI & 99m Tc-sestamibi \\
\hline MICU1 & mitochondrial calcium uptake protein 1 \\
\hline MiD49 & mitochondrial dynamics protein of 49 kDa \\
\hline MiD51 & mitochondrial dynamics protein of 51 kDa \\
\hline miR & microRNA \\
\hline mito-ROS & mitochondria-derived ROS \\
\hline MPI & myocardial perfusion imaging \\
\hline MPP & mitochondrial processing peptidase \\
\hline mtDNA & mitochondrial DNA \\
\hline NADH & nicotinamide adenine dinucleotide \\
\hline Ndufs2 & NADH dehydrogenase [ubiquinone] iron-sulfur protein 2 \\
\hline NFATc2 & nuclear factor of activated T-cells, cytoplasmic 2 \\
\hline NH3 & ${ }^{13}$-ammonia \\
\hline NCBI & National Centre for Biotechnology Information \\
\hline NLRP3 & NACHT, LRR and PYD domains-containing protein 3 \\
\hline NO & nitric oxide \\
\hline NOXA & $\begin{array}{l}\text { also known as PMAIP1, phorbol-12-myristate-13-acetate- } \\
\text { induced protein 1 }\end{array}$ \\
\hline NRF1 & nuclear respiratory factor 1 \\
\hline NT-proBNP & N-terminal pro B-type peptide \\
\hline O ${ }_{2}^{--}$ & superoxide anion \\
\hline OMM & outer mitochondrial membrane \\
\hline OPA1 & optic atrophy 1 \\
\hline OPTN & optineurin \\
\hline OXPHOS & oxidative phosphorylation \\
\hline PAAT & pulmonary arterial acceleration time \\
\hline
\end{tabular}




\begin{tabular}{|l|l|}
\hline PAEC & pulmonary arterial endothelial cells \\
\hline PAFib & pulmonary artery adventitial fibroblasts \\
\hline PAH & Pulmonary arterial hypertension \\
\hline PAP & pulmonary artery pressure \\
\hline PARL & presenillin-associated rhomboid-like \\
\hline PASMC & pulmonary artery smooth muscle cell \\
\hline PASP & pulmonary arterial systolic pressure \\
\hline PC & pyruvate carboxylase \\
\hline PDGF & platelet-derived growth factor \\
\hline PDH & pyruvate dehydrogenase \\
\hline PDK & pyruvate dehydrogenase kinase \\
\hline PERP & p53 apoptosis effector related to PMP-22 \\
\hline PFKFB3 & 6-phosphofructo-2-kinase/fructose-2,6-bisphosphatase 3 \\
\hline PGC-1 $\boldsymbol{\alpha}$ & peroxisome proliferator-activated receptor $\gamma$ coactivator 1- $\alpha$ \\
\hline PH & pulmonary hypertension \\
\hline PHD & prolyl hydroxylases \\
\hline PI3K & phosphatidylinositide 3-kinases \\
\hline PINK1 & PTEN-induced putative kinase \\
\hline PK & pyruvate kinase \\
\hline PKA & protein kinase A \\
\hline PKC $\boldsymbol{B}$ & protein kinase C $\delta$ \\
\hline PKMT & protein lysine methyltransferase \\
\hline PPAR $\gamma$ & peroxisome proliferator-activated receptor $\gamma$ \\
\hline PPHN & persistent pulmonary hypertension of the newborn \\
\hline RISP & Rieske iron-sulfur subunit \\
\hline RNAseq & RNA sequencing \\
\hline ROS & reactive oxygen species \\
\hline RVH & right ventricle hypertrophy \\
\hline SAB & pupra-aortic banding \\
\hline SGLT2 & sodium-glucose cotransporter 2 \\
\hline SIRT3 & sirtuin-3 \\
\hline SNP & single nucleotide polymorphism \\
\hline SOD2 & superoxide dismutase 2 \\
\hline STAT3 & signal transducer and activator of transcription 3 3 \\
\hline STfR & soluble transferrin receptor \\
\hline TASK-1 & acid-Sensitive Potassium Channel Protein-1 \\
\hline TAZ & tafazzin \\
\hline TFAM & transcription factor A mitochondrial \\
\hline TM & transmembrane \\
\hline
\end{tabular}




\begin{tabular}{|l|l|}
\hline TRPC6 & transient receptor potential channel 6 \\
\hline V/Q & ventilation to perfusion \\
\hline VDAC1 & voltage-dependent-anion-selective channel 1 \\
\hline VEGF & vascular endothelial growth factor \\
\hline VHL & von Hippel-Lindau gene \\
\hline XBP1s & X-box-binding protein 1 \\
\hline YAP & Yes-associated protein 1 \\
\hline
\end{tabular}




\section{Introduction}

Mitochondria are best known as the organelles that generate adenosine triphosphate (ATP, abbreviations summarized in Table 1) by means of oxidative phosphorylation (OXPHOS); however, energy production is but one of their key roles. Mitochondria also regulate oxygensensing, cell proliferation, apoptosis and mediate their own organellar quality control. Noncanonical functions of mitochondria, including production of reactive oxygen species (ROS), mitochondrial biogenesis, fission and fusion, mitophagy, and regulation of calcium homeostasis $(59,140,192,352)$ are highly relevant to the function of cells within the pulmonary circulation, in health and disease (8). Furthermore, new insights into the epigenetic regulation of mitochondria may explain susceptibility to cardiopulmonary diseases, such as pulmonary hypertension (PH).

Mitochondria evolved from primitive endosymbiont bacteria that had the ability for aerobic respiration. These bacteria formed an advantageous partnership with eukaryotic cells through a process called endosymbiosis $(106,165,274)$. During evolution, most of the bacterial proteins encoded by primitive bacterial DNA have been lost. Eukaryotic mitochondrial DNA, which resembles bacterial DNA, is maternally inherited (64). Modern mitochondrial DNA (mtDNA) is a small circular, polycistronic, $16 \mathrm{~Kb}$ DNA which encodes only 67 proteins, including 13 structural components of the electron transport chain (ETC) (7).

The adaptive ability to optimize systemic oxygen delivery in responses to changes in alveolar oxygen concentration (whether induced by disease or altitude) is a basic function of the pulmonary circulation. The pulmonary circulation actively vasodilates during the transition from fetal, hypoxic conditions to the oxygen-rich environment that exists with the onset of ventilation at birth 
(72). Postnatally, the lung autoregulates its regional blood supply to mitigate the effects of segmental airway hypoxia, caused by pneumonia and atelectasis, on systemic oxygenation. It does so by constricting smooth muscle cells (SMC) in the small pulmonary arteries (PA) serving hypoxic lung segments. This mechanism, called hypoxic pulmonary vasoconstriction (HPV) is intrinsic to the pulmonary arterioles (332). In the pulmonary circulation, mitochondria in PASMC function as oxygen sensors. They achieve this through their ability to vary production of diffusible ROS and alter the cytosolic redox state. Changes in ROS and the reduce/oxidized ratio of redox couples, such as NADH/NAD and NADPH/NADP, act as signalling molecules that target the fusion of vasoregulatory effector proteins, such as ion channels and kinases, reviewed in (333). Understanding pulmonary vascular oxygen sensing has relevance beyond physiology, since this pathway is disordered in pulmonary arterial hypertension $(\mathrm{PAH})(12)$.

Mitochondria are also the master regulators of cellular metabolism (304), including fatty acid oxidation, glucose oxidation and glutaminolysis. These metabolic pathways are dysregulated in PAH (271). The mitochondrial metabolic abnormalities described in PAH are similar to the metabolic abnormalities that were originally described in cancer by Otto Warburg (324). These acquired mitochondrial abnormalities increase uncoupled glycolysis and lactate production at the expense of glucose oxidation and pyruvate production. This Warburg metabolic shift contributes to disease pathophysiology by promoting hyperproliferation and apoptosis resistance of vascular cells (270). The Warburg phenomenon reflects a pseudohypoxic state, in which DNA methyltransferases (DNMT) (and other mechanisms) partially silence transcription of superoxide dismutase 2 (SOD2), decreasing hydrogen peroxide generation, thereby creating a hypoxia-like milieu that activates hypoxia inducible factor (HIF-1 $\alpha$ ). This HIF-1 $\alpha$ activation occurs despite the 
normal $\mathrm{PO}_{2}$ and thus is referred to as pseudohypoxia. HIF-1 $\alpha$ in turn upregulates expression of pyruvate dehydrogenase kinase (PDK), which phosphorylates and inhibits pyruvate dehydrogenase (PDH), a key OXPHOS enzyme. The PDK-induced Warburg shift is reinforced by an epigentically driven increase in the expression of proglycolytic splice variant change in pyruvate kinase (PK) isoform expression, specifically an increase in the PKM2/PKM1 ratio.

Mitochondria normally exist in filamentous networks but in PAH they are fragmented, an emerging hallmark of PAH that reflects an imbalance of mitochondrial fission/fusion (favouring fission). This fission/fusion imbalance is caused in large part by activation of dynamin related protein 1 (Drp1), through post-translational Drp1modification (192), upregulation of its binding partners (MiD49 and MiD51) (59), and downregulation of mitofusin-2 (273). The division of mitochondria must be coordinated with nuclear division or else a cell cycle checkpoint is violated and the cell dies. The rate of mitotic fission is elevated in PAH, favouring rapid cell cycle progression and apoptosis resistance. Comparable changes in mitotic fission occur by similar mechanisms in cancer (259) and in both conditions this pathway offers therapeutic targets to reduce pathological rates of cell proliferation and apoptosis resistance, as reviewed in (9).

Finally, mitochondria regulate intramitochondrial calcium concentration and serve as buffers for elevated cytosolic calcium concentrations. The mitochondrial calcium uniporter complex (MCUC), an inwardly rectifying, $\mathrm{Ca}^{2+}$-selective, ion channel in the inner mitochondrial membrane (IMM), is the channel through which calcium enters the mitochondria (158). MCUC downregulation is a unifying mechanism linking abnormal metabolism and dynamics in PAH (140). Increases in microRNA (miR)-25 and miR-138-3p in PAH patients downregulate the 
expression of a MCUC pore-forming subunit called MCU and increase in the expression of an inhibitory subunit, called mitochondrial calcium uptake protein 1 (MICU1). Together these changes inhibit mitochondrial calcium uptake, resulting in elevated cytosolic calcium (which promotes vasoconstriction and fission). MCUC inhibition also reduces intramitochondrial calcium, which inhibits several of the enzymes that are curcial to oxidative metabolism, scuh as PDH. The MCUC and its epigenetic regulators have diagnostic and therapeutic relevance in PAH (140). We will elaborate on the role of the MCU complex in metabolism in more detail elsewhere in this review.

In this review, we will discuss the multiple regulatory functions of mitochondria in normal lung homeostasis, focusing mainly on HPV. We will discuss the role of mitochondrial dysfunction in pulmonary vascular diseases, focusing on $\mathrm{PAH}$. We will also highlight roles of metabolic pathways, mitochondrial dynamics and epigenetic mechanisms affecting the cells of the pulmonary vasculature in both health and disease. Finally, we summarize some newer applications of nuclear imaging for the diagnosis of pulmonary vascular diseases and review clinical trials of pharmacological agents that target mitochondrial metabolic pathways. Newly recognized mechanisms that dysregulate mitochondrial metabolism, dynamics, and calcium homeostasis in PAH suggests new disease biomarkers and imaging modalities. These novel epigenetic and mitochondrial pathways offer potential therapeutic strategies, including inhibitors of PDK (dichloroacetate), activators of the Randle cycle (such as the partial fatty acid oxidation inhibitors, trimetazidine and ranolazine), PKM isoform modulators (shikonin), miR mimics and anti-miRs, inhibitors of Drp1 and its binding partners, and mitofusin-2 augmentation. To the greatest extent possible, we have invited major contributors to the field to include a key figure from their research 
in the review, or have reproduced their key figures to try and achieve balance.

\section{Function of mitochondria in the lung: Oxygen sensing}

The ability to detect and respond to changes in environmental oxygen tension facilitates human life on Earth. From fetal development, through birth, and subsequent challenges by disease (such as pneumonia) and environmental changes (such as high altitude), optimized oxygen uptake by the lungs is a requisite for optimal systemic oxygen delivery and survival. Humans begin life in a hypoxic intrauterine environment, receiving oxygen from the mother through placental blood flow. The fetal pulmonary vascular circulation is constricted and is a high-resistance, low-flow circuit that is largely bypassed until birth. Blood returning to the fetal right heart reaches the left heart by shunts, either across the foramen ovale or through the fetus' patent ductus arteriosus. At birth, with the first breath, the ductus constricts and the pulmonary vasculature simultaneously dilates. When combined with the inflation of the lungs, oxygen-induced pulmonary vasodilatation lowers the resistance to pulmonary vascular perfusion and, coupled with ductal constriction/obliteration and foraminal closure, antegrade flow of blood from the right ventricle is diverted to the pulmonary circulation (71). After birth, humans usually live in an environment replete with abundant oxygen, however we may be exposed to hypoxia as a consequence of changes to our environment (such as ascent to high altitude $(30,151))$ or through disease (such as acute lung injury $(217)$, chronic lung disease $(217,260)$, pneumonia $(177)$ or atelectasis $)(1,125,148)$.

The Homeostatic Oxygen Sensing System (HOSS) is an elegant network of specialized tissues (19), that sense oxygen in their local environments and respond in sensor-specific ways that change vascular tone, ventilation or catecholamine secretion to optimize systemic oxygen delivery. The HOSS is comprised of type 1 (glomus) cells in the carotid body, PASMC, fetoplacental arteries in 
the placenta, the ductus arteriosus (DA), adrenomedullary chromaffin cells of the adrenal glands, and neuroepithelial bodies, a type of neuroendocrine cell in the airways (Figure 1). Most of these specialized tissues use a redox-based oxygen sensor (most often based within mitochondria (85, 97, 333)) to regulate the function of ion channels (usually potassium and calcium channels (198, 332)). Upon sensing small decreases in inspired or circulating oxygen concentrations, an orchestrated effector response in the HOSS is initiated to compensate and optimize both the uptake and delivery of oxygen. For example, the type 1 cells of the carotid body increase ventilation to enhance oxygen uptake, PASMC in small resistance-level pulmonary arteries constrict to match ventilation to perfusion in the lung to avoid perfusing hypoxic lung segments, ductus arteriosus SMC constrict in response to elevated $\mathrm{PO}_{2}$, diverting blood to newly ventilated lungs, and the fetal adrenomedullary chromaffin release catecholamines to counteract hypoxic stress at birth, reviewed in (333).

\section{Hypoxic pulmonary vasoconstriction (HPV)}

Identified in 1894 by Bradford and Dean (39), HPV was first characterized in detail by von Euler and Liljestrand, who described the opposing hypoxic responses of the feline pulmonary (constriction) and systemic (dilatation) circulations (93). HPV is a mechanism intrinsic to the lung that matches ventilation to perfusion (V/Q) in order to optimize systemic oxygen delivery. V/Q matching is achieved via the constriction of small, intrapulmonary, arteries in response to alveolar hypoxia (186). HPV can be global (in response to environmental hypoxia), inducing a rise in pulmonary artery pressure (PAP), or localized, as elicited by atelectasis or pneumonia (211). In the latter case, both the hypoxia and HPV are localized to a segment or lobe of the lung and blood is diverted from this hypoxic region to a better-oxygenated portion of the lung with no significant 
effect on PAP, as seen in (Figure 2).

The onset of HPV is rapid (occurring within seconds of exposure to hypoxia), and constriction reaches a maximum intensity within minutes $(29,149)$. Though modified by the endothelium, the core mechanism of HPV is intrinsic to the PASMC (333). HPV is rapidly reversible upon restoration of normal airway oxygen levels, unless $\mathrm{PH}$ or other adverse vascular remodeling has occurred. The reversibility of sustained HPV has been illustrated by Grant et al (114). A patient with an endobronchial adenoma exhibited longstanding left lung atelectasis and a matching V/Q abnormality, both of which were reversed after removal of the adenoma (114). The resistance pulmonary arteries are unique in their vasoconstrictor response to hypoxia (286). The systemic vasculature, including renal, mesenteric and cerebral arteries, dilates in response to hypoxia. This systemic dilatation also serves to increase tissue oxygen delivery $(187,209,357)$. The unique localization of HPV to resistance PASMC relates in part to spatially heterogenous mitochondrial function (207) and ionic diversity (210). The resistance PASMC have mitochondria that uniquely sense physiologic hypoxia and respond with dynamic changes in production of reactive oxygen species (ROS) and are enriched in oxygen-sensitive, potassium channels, including voltage-gated channels (Kv).

While increased by endothelium-derived vasoconstrictors (e.g. endothelin and thromboxane) and inhibited by endothelium-derived vasodilators (e.g. nitric oxide and prostacyclin) the core effector mechanism of HPV resides in the PASMC, reviewed in (332). HPV is triggered by a mitochondrial redox signal described below, which coordinates the response of voltage- and redox-sensitive potassium and calcium channels $(332,333)$. Briefly, in normoxia the open state probability of 
voltage-gated potassium channels (e.g. Kv1.5, Kv2.1) [and other channels such as classical transient receptor potential channel 6 (TRPC6) (335)] results in tonic leak of $\mathrm{K}^{+}$out of the PASMC [along its concentration gradient $(145 / 5 \mathrm{mM}$, inner/outer)]. This maintains the cell's resting membrane potential near $\sim-60 \mathrm{mV}$. This negative membrane potential decreases the open state probability of voltage-gated, L-type calcium channels and maintains a state of relaxation. During hypoxia, outward potassium current $\left(\mathrm{I}_{\mathrm{K}}\right)$ is inhibited, which depolarizes the cell membrane and increases the open state probability of calcium channels, causing an influx of $\mathrm{Ca}^{2+}$ into the cell down the $\mathrm{Ca}^{2+}$ gradient. This extracellular:intracellular gradient is approximately 20,000:1. This rise in cytosolic calcium, and a subsequent Rho kinase-mediated calcium sensitization (94), induces contraction of the PASMC (201, 247).

Identification of the key voltage-gated channels responsible for HPV was achieved using pharmacological and electrophysiology experimentation using the patch clamp technique and transgenic mice lacking certain $\mathrm{Kv}$ channels. Interrogation of isolated PASMC from resistance PAs identified $\mathrm{Kv}$ as well as large conductance, voltage-gated calcium channels $\left(\mathrm{Ca}_{\mathrm{L}}\right)$ in the effector mechanism of HPV $(21,247)$. Blocking Kv channels with 4-aminopyridine (4-AP) elicits pulmonary vasoconstriction mirroring HPV in isolated perfused rodent lungs (129). Conversely, blocking the $\mathrm{Ca}_{\mathrm{L}}$ channel with either nifedipine or verapamil substantially inhibits hypoxiainduced vasoconstriction, whilst the $\mathrm{Ca}^{2+}$-channel agonist BAY K8644 significantly enhances HPV (200, 201). Molecular identification of the specific ion channels involved in HPV has revealed central roles for Kv1.5 (KCNA5), and Kv2.1 $(18,101)$. For example, the Kv1.5 knockout mouse has markedly reduced HPV (14). It appears that heteromeric channels comprised of several types of subunits, such as Kv1.2/Kv1.5 and Kv2.1/Kv9.3 may be particularly sensitive to hypoxia 
(94).

There is evidence supporting a role for other classes of $\mathrm{K}^{+}$and $\mathrm{Ca}^{2+}$ channels in the effector mechanism of HPV (325), including two-pore $\mathrm{K}^{+}$channels $\left(\mathrm{K}_{2 \mathrm{P}}\right)(87,107)$ and transient receptor potential cation channel subfamily $\mathrm{C}$ member 6 (TRPC6) (335). $\mathrm{K}_{2 \mathrm{P}}$ channels, such as acidsensitive Potassium Channel Protein-1 (TASK-1), are active at very negative membrane potentials in PASMC, making them plausible targets for initiating HPV (122). However, pharmacological inhibition of these channels does not result in pulmonary vasoconstriction (107) and recent studies using a TASK-1 knockout mouse indicate this channel does not mediate HPV (216). In addition to the L-type $\mathrm{Ca}^{2+}$ channel, $\mathrm{Ca}^{2+}$-induced $\mathrm{Ca}^{2+}$ release also contributes to HPV. HPV also reflects calcium sensitization, reviewed in (326). Interestingly, effector mechanisms are similar in pulmonary and systemic circulations in that both arterial beds constrict in response to $\mathrm{K}^{+}$channel inhibitors and relax in response to calcium channel blockers (247). Therefore, it appears the opposing, tissue-specific responses to hypoxia result primarily from differences in oxygen-sensor (mitochondrial) function (207). The link between specific ion channels and mitochondria likely relates to the mitochondria's ability to produce ROS and alter levels of electron donors, such as NADH, NADPH and reduce glutathione. Electron donors serve as redox signaling molecules and can diffuse from the mitochondria and interact with redox-sensitive amino acids in key regions of oxygen-sensitive ion channels. Changes in reduction and oxidation of the channels controls channel gating and also can alter channel expression, reviewed in (275). For example, Kv1.5 has six redox sensitive cysteines that regulate its expression and function (305).

\section{Oxygen-sensing: molecular mechanism}


Oxygen is a key player in a complex cascade of reduction-oxidation (redox) reactions within the mitochondrion. The primary purpose of respiration is provision of molecular oxygen to the mitochondria. $\mathrm{O}_{2}$ is the terminal electron acceptor for the mitochondrial electron transport chain, ETC, a multi complex redox cascade. At the distal end of the ETC, oxygen receives electrons and is chemically reduced, forming water. The electrons that will reduce oxygen to water originate from electron donors, nicotinamide adenine dinucleotide (NADH) and flavin adenine dinucleotide $\left(\mathrm{FADH}_{2}\right)$, which are themselves products of mitochondrial metabolic cycles, such as Krebs' cycle. These donors pass their electrons down a redox potential gradient across 4 mega-complexes (ETC Complexes I-IV) that span the inner mitochondrial membrane. Electron transport powers the pumping of hydrogen ions across the inner mitochondrial membrane, generating an electrochemical gradient that powers ATP synthase (214). Thus in the mitochondrial oxygen sensor we see the coordinated linkage between oxygen supply, metabolism and energy production The sensor mechanism of $\mathrm{HPV}$ reflects $\mathrm{PO}_{2}$-sensitive production of redox signaling molecules that originate as a by-product of physiological electron flux $(13,19,51,86,123,211,215,235,325$, $329,331,332)$. A small proportion (1-3\%) of electron flux) is uncoupled and generates superoxide anion $\left(\mathrm{O}_{2}{ }^{-}\right) \cdot \mathrm{O}_{2}{ }^{-}$is rapidly converted by superoxide dismutase 2 (SOD2) to hydrogen peroxide $\left(\mathrm{H}_{2} \mathrm{O}_{2}\right) . \mathrm{H}_{2} \mathrm{O}_{2}$ serves as a diffusible redox signaling molecule and can modulate the activity of redox-sensitive ion channels and enzymes and $(209,211)$. Mammalian oxygen-sensing and the role of mitochondria-controlled redox chemistry has been reviewed in (333).

While there is agreement that mitochondria act as oxygen-sensors and that ETC-derived ROS alter effector mechanisms that mediate vasoconstriction $(13,17,235)$, controversy remains regarding both the precise molecular identity of sensor subunits within the mitochondrial redox sensor (51, 
$86,123,292)$, and whether the sensor output is an hypoxic rise or an hypoxic fall in $\mathrm{ROS} / \mathrm{H}_{2} \mathrm{O}_{2}$ levels $(51,123,325,327,329,331)$. The oxygen sensor itself has been variably proposed to reside in ETC Complex I $(17,86)$ also (16), Complex III $(51,123)$, and Complex IV (292). Schumacker et al. identified the Rieske iron-sulfur subunit (RISP) of Complex III as the oxygen-sensor (328) and demonstrated that knockout of RISP attenuated hypoxia-induced ROS production (measured using compartment-specific fluorescent redox probes) and subsequent HPV (Figure 3A). However, most recently, NADH dehydrogenase [ubiquinone] iron-sulfur protein 2 (Ndufs2), the quinone binding site in ETC Complex I (and interestingly, not the RISP) has been characterized as the mitochondrial oxygen sensor in both the carotid body (97) and the PASMC (86). The latter study showed that Ndufs2 is critical to the acute hypoxic response, mediating a hypoxic decrease in intramitochondrial and cytosolic $\mathrm{H}_{2} \mathrm{O}_{2}$. Normal function of Ndufs2 is required for hypoxiainduced increases in cytosolic calcium. The mechanism by which Ndufs 2 senses changes in $\mathrm{PO}_{2}$ was determined to be redox-based, an observation concordant with previous predictions $(13,17$, 19, 257). Lungs harvested from mice exposed to acute hypoxia (10\% oxygen) had a greater abundance of reduced Ndufs 2 thiols, further suggesting that hypoxia is a state of reduction, whereas normoxia is a state of oxidation (86). Importantly, airway nebulization of small inhibitory RNA targeting Ndufs2 (siNdufs2) reduced lung Ndufs2 expression and substantially inhibited HPV in vivo in rats. The current understanding of the mechanism of pulmonary vascular oxygensensing is summarized in (86), (Figure 3B-E). The identification of a specific ETC subunit as the oxygen-sensor provides a new target of investigation in diseases of impaired oxygen-sensing, including pulmonary hypertension $(\mathrm{PH})$. The mitochondrial phenotype in pulmonary vascular diseases is discussed in later sections of this review; however, ETC composition, and the function of specific ETC subunits, is accepted to be critical to pulmonary vascular oxygen sensing. In this 
regard manipulation of NDUFS2 structure, function and expression may be relevant not only to oxygen sensing, but to human syndromes such as pulmonary hypertension.

Regarding ROS production/levels during acute hypoxia, one hypothesis proposes that hypoxia increases ROS and reflects autoxidation of the ETC, due to inhibition of the distal ETC (as seen in Figure 3A) $(327,329)$. In contrast, our group finds that ROS production in PASMC is directly proportional to $\mathrm{PO}_{2}$, decreasing as $\mathrm{PO}_{2}$ falls $(17,19,20,86)$. Extensive experimental data using multiple ROS chemical and molecular probes (including several that measure intramitochondrial $\mathrm{H}_{2} \mathrm{O}_{2}$ ) demonstrate a decrease in PASMC mitochondrial ROS within seconds of exposure to moderate, physiologic hypoxia. This reduced ROS production reflects a reduced rate of electron flux caused by reduced availability of the terminal electron acceptor (molecular oxygen) $(86,333)$. Consistent with the finding that $\mathrm{ROS}$ are produced in proportion to $\mathrm{PO}_{2}, \mathrm{ROS}$ levels rise in ductus arteriosus $\mathrm{SMC}$ at birth as $\mathrm{PO}_{2}$ increases $(20,309)$. Likewise, ROS levels are low in cardiac myocytes during ischemia and increase with reoxygenation, during the reperfusion phase of myocardial ischemia-reperfusion injury (134). These divergent hypotheses regarding mitochondrial ROS in acute HPV are summarized in Figure 4 (330).

It is important to note that experimental design diverges between groups and that experimental conditions (stated or unstated) can vastly change findings. Since HPV onsets in seconds at physiologic levels of hypoxia and is fully reversible, experiments should focus on the first minutes of onset of HPV elicited by moderate hypoxia (i.e. PO2 of 40-50 mmHg). In experiments where conditions include moderate hypoxia (not anoxia) and physiologic $\mathrm{pH}$ (7.35-7.45) during the hypoxic exposure there is robust HPV (e.g. a 50-100\% increase in pulmonary vascular resistance 
in ex vivo and in vivo preclinical models). In such experiments there is a consistent finding that ROS levels are reduced, and this fall in ROS production precedes the rise in intracellular calcium which in turn precedes the onset of vasoconstriction. Extreme hypoxia (bordering on anoxia) and $\mathrm{pH}$ fluctuations can both lead to increased ROS production, via reoxygenation injury and uncontrolled acidosis, respectively. Additionally, since HPV is a rapid response that is sustained over time, it likely exists in multiple phases, beginning with a rapid onset phase where ROS are decreased, followed by sustained constriction where ROS from other sources may (or may not) increase. It is therefore incumbent on all research groups to carefully control $\mathrm{pH}$ and oxygenation, monitor the magnitude of the acute hypoxic pressor response and its reversibility and specify the phase of HPV their experimental design considers. In this regard, the evidence for complex I and NDUFS2 satisfies all the preceding criteria and loss of this single component of the ETC reduces robust HPV and attenuates a fall in ROS with hypoxia. It is our view that the evidence for complex III and an increase in ROS is weaker in that much of the data are cellular (not reproduced in in vivo) and experimental conditions of $\mathrm{pH}$ etc. are less well described $(51,123)$. In addition, redox measurements are made somewhat later in the exposure to hypoxia (rather than in the first 0-10 minutes as in the NDUFS2 experiements). In the case of Complex IV (292), it is our view that the extremely modest rise in PA pressure the authors observed with hypoxia $(1 \mathrm{mmHg})$ is small even for mice and may not reflect robust HPV. This raises some question as to the generalizability of these findings.

\section{Clinical applications of HPV}

HPV is exploited surgically to reduce bleeding while also optimizing oxygenation in patients undergoing lung surgery. Single-lung anesthesia (or "one lung ventilation") is used for lung tumor 
resections, pneumonectomy, and other thoracic procedures (220). An example of this procedure is outlined in Figure 5, in a patient undergoing resection of an endobronchial mass underwent singlelung anesthesia. Using a double-lumen tube, the patient is intubated and both lungs are ventilated. Then the airway serving the operative lung is occluded causing it to collapse; meanwhile the nonoperative lung is selectively ventilated. HPV within the collapsed operative lung reduces its perfusion which minimizes bleeding while also decreasing shunting and systemic hypoxemia.

Hypothermia, certain anesthetics, and vasodilators can inhibit HPV. It is therefore important to choose the appropriate anesthetic and maintain a patient's core body temperature during singlelung anesthesia. Historically, HPV enhancement has been achieved using low-dose almitrine, a respiratory stimulant. During single-lung ventilation, adding almitrine $\left(4 \mu \mathrm{g} \mathrm{kg}^{-1} \mathrm{~min}^{-1}\right)$ to inhaled nitric oxide (NO) increases HPV and improves systemic oxygenation (287). However, almitrine has since been withdrawn from clinical use because it can cause peripheral neuropathy, but is nonetheless proof of principle that enhancing HPV improves V/Q matching and systemic oxygenation.

\section{Impaired oxygen-sensing in chronic hypoxia - normoxic activation of HIF-1 $\alpha$}

There is an interesting intersection between oxygen-sensing in the normal pulmonary circulation and the pathologic processes that drive PAH. This intersection is perhaps best seen in Chuvash disease patients (115), and in fawn hooded rats (FHR) (37). Both Chuvash patients and the FHR spontaneously develop pulmonary hypertension, in part because their oxygen-sensing system inappropriately signals a hypoxic environment when none exists. This pseudohypoxic response is an important part of the pathogenesis of $\mathrm{PAH}$. 
Named for the mid-Volga River region of Russia, patients with Chuvash disease have enhanced HPV, polycythemia and pulmonary hypertension, despite normal inhaled oxygen concentrations (7). Chuvash disease is characterized by a homozygous missense mutation in the von HippelLindau (VHL) gene (VHL 598C-T). This mutation impairs the VHL's ability to interact with $\alpha$ subunits of the transcription factors, hypoxia inducible factors $1 \alpha$ and $2 \alpha$ (HIF- $1 \alpha$ and HIF-2 $\alpha$ ). Under normoxic conditions, HIF proteins are hydroxylated by oxygen-sensitive prolyl hydroxylases (PHDs), which subsequently mark them for ubiquitination by VHL, which targets them for proteasomal degradation. During hypoxia, HIF proteins are not hydroxylated, and are therefore not targeted by VHL and are not degraded. This stabilizes HIF-1 $\alpha$ which allows it to move to the nucleus where it mediates transcriptional programming. The VHL mutation in Chuvash disease impairs the ability of VHL to interact with HIF, ultimately preventing HIF degradation and triggering the hypoxic cascade during normoxia (111). This permits transcription of HIF-regulated genes whose expression during normoxia is pathological, such as erythropoietin (7, 139, 293), glucose transporter 1 (Glut1), and vascular endothelial growth factor (VEGF) (7). Thus, patients with Chuvash disease behave as if they were exposed to chronic hypoxia, despite normal environmental oxygen tension. The HIF pathway is also redox-regulated and critical to oxygen-sensing (154); however, since this pathway involves transcriptional activity, its effects are much slower to onset than the ion channel-initiated vascular response to acute hypoxia (e.g. HPV). Likewise, FHR, a pre-clinical model of spontaneous pulmonary hypertension, have normoxic activation of HIF-1 $\alpha$, and develop PAH and polycythemia. In FHR, VHL is functional, rather it is an epigenetic reduction in expression of mitochondrial SOD2 (the mitochondrial enzyme that generates $\mathrm{H}_{2} \mathrm{O}_{2}$ ) that leads to redox-mediated HIF-1 $\alpha$ activation (16). 


\section{Pulmonary hypertension and vascular remodeling}

Pulmonary hypertension $(\mathrm{PH})$ is a life-threatening syndrome in which pulmonary arterial pressure is elevated, usually resulting in dyspnea and limitations in exercise performance. PH eventually culminates in right ventricular failure. PH is increasing in incidence and prevalence (338). Although defined by the hemodynamic criteria of resting mean pulmonary arterial pressure (mPAP) $\geq 20 \mathrm{~mm} \mathrm{Hg}$, the etiology and clinical presentation of $\mathrm{PH}$ is quite diverse (288). The World Health Organization recognizes 5 groups of $\mathrm{PH}$ : 1) $\mathrm{PAH}$ and congenital heart disease (in which the pathology is primarily in the pulmonary vasculature); 2) PH secondary to left heart disease; 3) $\mathrm{PH}$ due to chronic lung diseases or hypoxemia; 4) chronic thromboembolic $\mathrm{PH}$ (CTEPH); and 5) PH due to unclear multifactorial mechanisms, such as hemolysis or sarcoidosis. Given the numerous causes of $\mathrm{PH}$, a considerable heterogeneity exists in the mechanisms that cause the disease (102). Since most studies of the role of mitochondria in the pulmonary vasculature were performed in Group $1 \mathrm{PH}$ (known as $\mathrm{PAH}$ ), we have focused this section on Group 1 PH.

$\mathrm{PAH}$ is characterized by profound, obstructive remodeling of the pulmonary vasculature, particularly evident in small, intrapulmonary arteries and arterioles (Figure 6). In PAH there is stiffening, obstruction and constriction of the pulmonary arteries. The arterial wall is composed of three layers: intima, media, and adventitia. Each layer contains an assortment of cell types, each with its own specific functional characteristics (199). Each layer contributes uniquely to the development of PAH. The intima and media, along with their principle cellular components, the pulmonary arterial endothelial cells (PAECs) and pulmonary arterial smooth muscle cells (PASMCs), respectively, have received much attention. In contrast, the adventitia and its principal 
cell type, the fibroblast, have been somewhat overlooked. All layers of the pulmonary vessel wall are involved in adverse vascular remodelling in PAH. Pathologic changes include endothelial dysfunction leading to intimal hyperplasia, medial hypertrophy and hyperplasia (due to proliferation of PASMC) with distal migration of PASMC into usually non-muscular intra-acinar PAs, adventitial fibrosis (due to fibroblast proliferation and increased collagen production), and infiltration of inflammatory cells and progenitor cells (142).

\section{Pathologic and therapeutic implications of dysregulated mitochondrial metabolism in PAH}

In PAH, changes in mitochondrial metabolism have been observed in all cell types within the pulmonary arterial wall, including PAEC, PASMC and pulmonary artery adventitial fibroblasts (PAFib). Each of these cells exhibit changes in one or more mitochondrial metabolic pathways, i.e., altered aerobic glycolysis or fatty acid oxidation (FAO) and/or induction of glutaminolysis (Table 2a-c). While aerobic glycolysis is well studied, there is only one study on FAO in PAEC, two on glutaminolysis in PAEC, one on FAO in PASMC, and one on glutaminolysis in PASMC. No study has explored FAO or glutaminolysis in PAFib. Changes in mitochondrial metabolism are associated with altered mitochondria-derived ROS (mito-ROS) production, mitochondrial membrane potential $\left(\Delta \psi_{m}\right)$ and mitochondrial morphology (Table 2a-c). Interestingly, some forms

of metabolic remodeling (notably Warburg metabolism-an increase in uncoupled glycolysis) is shared by all 3 cell types. The consequences of this metabolic shift is that they become more apoptosis-resistant and proliferative $(12,45,173)$, likely contributing to the observed obstructive vasculopathy.

In PAH, a shift from oxidative phosphorylation to uncoupled aerobic glycolysis is observed (11, 
176). This metabolic phenotype favours cell proliferation and reduces apoptosis in cancer cells (35) and PAH cells $(258,304)$. One of the main causes of increased, uncoupled, aerobic glycolysis in PAH is PDH inhibition caused by increased PDK expression and/or activity. PDH, which catalyzes the conversion of pyruvate to acetyl-CoA, is the rate-limiting enzyme in glucose oxidation (240); PDK phosphorylates and inhibits PDH $(139,349)$. Therefore, upregulation of PDK results in decreased oxidative phosphorylation. In humans, there are four isoforms of PDK (PDK1-4) and all are inhibited by dichloroacetate (DCA), a potential metabolic therapeutic agent for PAH (205), as will be discussed.

\section{Pulmonary artery endothelial cells}

Several studies have observed a shift from glucose oxidation to aerobic glycolysis in PAEC. For example, Xu et al. studied the cellular bioenergetics in PAEC and showed that endothelial cells derived from patients with idiopathic PAH (IPAH) have increased glucose uptake, lactate production, and decreased oxygen consumption (345). This metabolic shift in PAEC was associated with decreased complex IV activity and reduced SOD2 expression. They postulated the loss of SOD2 contributes to HIF-1 $\alpha$ activation, similar to our findings in PASMC derived from IPAH patients and FHR $(15)(98,345)$.

Unlike the activation of HIF-1 $\alpha$ elicited in PAH PASMC by reduced ROS, others have found activation of HIF- $1 \alpha$ to be the result of increased levels of mito-ROS, induced by the endothelin1 (ET-1)-mediated translocation of endothelial nitric oxide synthase (eNOS) from the plasma membrane to the mitochondria via protein kinase $\mathrm{C} \delta(\mathrm{PKC} \delta)$ (300). The increase in ET-1 also decreases mitochondrial membrane potential, $\Delta \psi_{m}$. Rabinovitch et al. showed that mice with a 
PAEC specific mutation of bone morphogenetic protein receptor type 2 (BMPR2), the most common mutation in heritable PAH (HPAH), developed PH in hypoxia which was not reversed upon reoxygenation (83). This $B M P R 2$ mutant mouse model of PAH is associated with reduced expression of several mitochondrial regulators [p53, peroxisome proliferator-activated receptor $\gamma$ coactivator 1- $\alpha$ (PGC-1 $\alpha)$ and TFAM], recapitulating the phenotype of PAH patients with BMPR2 mutation. In aggregate, these abnormalities lead to mitochondrial dysfunction, manifesting as mitochondrial depolarization, excess mitochondrial fission and increased apoptosis in both BMPR2 mutant mice and PAH patients (83). Decreased mito-ROS production was observed only in $B M P R 2$ mutant mice studied under hypoxia. In contrast, when studied in normoxia, PAEC from BMPR2 mutant mice and PAH patients exhibit increases in $\mathrm{p} 53, \mathrm{PGC}-1 \alpha$ and TFAM and display mitochondrial hyperpolarization, increased mito-ROS (only in BMPR2 mutant mice), excess mitochondrial fission, increased glycolysis and increased inflammation (83). This study demonstrated the differential involvement of $B M P R 2$ mutation in normoxia (increased inflammation) versus hypoxia-reoxygenation (increased apoptosis, and induction of hypoxic $\mathrm{PH}$ ).

Recently, Caruso et al. studied the miR and proteomic profiles of blood outgrowth endothelial cells (BOEC) from HPAH patients with BMPR2 mutations and IPAH patients. They found that the downregulation of miR-124 in both BOEC populations increased expression of the splicing factor polypyrimidine-tract-binding protein $(P T B P 1)$, resulting in alternative splicing of PK muscle isoforms 1 and 2 (PKM1 and 2), and subsequent increased PKM2 expression. The proglycolytic PKM2 isoform predominance enhanced uncoupled, aerobic glycolysis and increased lactate production while also decreasing translocation of pyruvate to mitochondria (45). Thus, in PAECs, acquired mitochondrial abnormalities, due to alterations in the miR-124/PTBP1/PKM1/2 pathway 
and BMPR2 deficiency, contribute to disorders of mitochondrial metabolism and dynamics that are relevant to the proliferation/apoptosis imbalance in these cells.

Cao et al. found that increased 6-phosphofructo-2-kinase/fructose-2,6-bisphosphatase 3 (PFKFB3) expression/activity in PAEC (isolated from hypoxic mice and IPAH patients), also promotes glycolysis (43). PFKFB3 increases the production of growth factors (platelet derived growth factor [PDGFB], fibroblast growth factor 2 [FGF2]) and production of proinflammatory cytokines, such as $\mathrm{C}-\mathrm{X}-\mathrm{C}$ motif chemokine 12 (CXCL12) and interleukin $1 \boldsymbol{\beta}$ (IL1 $\beta)$, via a HIF-2 $\alpha$ dependent mechanism. Reducing PFKFB3, by genetic deletion or using a PFKFB3 inhibitor, slowed PH progression in hypoxic mice and improved pulmonary vascular remodeling in Sugen/Hypoxia PAH rats (evident as reduced PA wall thickness, decreased percentage of muscularized vessels and reduced endothelial cell proliferation)(44). This reinforces the pathologic nature of increased uncoupled glycolysis in PAECs in PAH.

There is a reciprocal relationship between glucose oxidation and FAO such that when one increases the other decreases. This is called the Randle cycle and we have previously shown that in right ventricle hypertrophy (RVH), partial inhibition of FAO (using trimetazidine or ranolazine) is sufficient to increase glucose oxidation and improve cardiac function (96). However, we did not explore the effects on pulmonary vascular cells. While no study has explored PAEC FAO metabolism in PAH, Singh et al. examined the healthy human PAEC under hypoxia and found that PAEC display a decrease in glucose oxidation and this is due to an increase of fatty acid synthase (FAS) (290), which in turn upregulates HIF-1 $\alpha$, Glut1, hexokinase II (HK2) and decreases PDH activity, resulting in a shift to glycolysis and FAO (290). Moreover, increased expression of FAS 
causes apoptosis resistance by decreasing Bax and increasing Bcl-2, increases autophagy via reducing p-mTOR and p62, and increases VEGF-dependent angiogenesis (290). The role of Randle's cycle in PAECs requires further exploration to determine whether it contributes to adverse vascular remodeling in PAH.

Glutaminolysis was initially studied in experimental RVH. Like most metabolic abnormalities in $\mathrm{PAH}$, it is a pathway that is also upregulated in cancer. While the RV does not normally utilize glutaminolysis, this pathway, which is relevant both to bioenergetics and hypertrophy, is upregulated in monocrotaline (MCT)-induced RVH (245). We further showed that treatment of the glutamine antagonist 6-diazo-5-oxo-L-norleucine (DON) in MCT rats increased PAAT, reflecting a reduction in pulmonary vascular disease. This indirectly suggested that there is also upregulated, pathologic glutaminolysis in the pulmonary vasculature in PAH (245). Two studies have subsequently examined the role of glutaminolysis in PAH PAEC. In the first study, the authors found that vascular stiffness activates glutaminolysis and glycolysis by a Yes-associated protein 1 (YAP) and tafazzin (TAZ) (or WWRT1)-dependent mechanism (33). Hallmarks of pathway activation include increased expression of glutaminase (GLS1), lactate dehydrogenase A (LDHA) and pyruvate carboxylase (PC). Egnatchik et al. found substantial pulmonary vascular glutamine uptake in PAH patients with BMPR2 mutations and confirmed the occurrence of increased glutamine uptake in PAEC isolated from $B M P R 2$ mutant mice. In $B M P R 2$ mutant mice the increase in glutaminolysis was due to the loss of sirtuin-3 (SIRT3) activity and the activation of HIF-1 $\alpha$ (88). These studies suggest that glutaminolysis inhibition may be a potential therapeutic strategy in PAH (with benefits to the pulmonary vasculature and right ventricle). 


\section{Pulmonary artery smooth muscle cells}

There is a shift in glucose metabolism from oxidation to uncoupled, aerobic glycolysis in PASMC derived from FHR and IPAH patients. In FHR, which are created by interbreeding of rat strains, the Warburg metabolic shift is due to lung-specific increases in DNA methylation (lung-specific increased expression of DNMT1 and DNMT3b). Although there are likely broad methylomic changes, we documented hypermethylation of a CPG island in the SOD2 promoter which partially inhibits gene transcription. In addition, these rats have deficient expression of complex I/III (15, 36). In aggregate, these acquired abnormalities decrease mito-ROS production, creating a condition of pseudohypoxia in PASMC, marked by pathological HIF-1 $\alpha$ activation. HIF-1 $\alpha$ is known to activate PDK, which inhibits glucose oxidation and promotes a reliance on uncoupled glycolysis. Dysregulation of complex I/III likely contributes to mitochondrial hyperpolarization and decreased mito-ROS production, although the precise mechanism by which this occurs remains uncertain. The effect of activation of HIF-1 $\alpha$ on glycolysis in PASMC has subsequently been confirmed in PASMC from MCT and Sugen-hypoxia rats. In these PASMC, the expression of Glut1, HK1, and HIF-1 $\alpha$ are increased due to increased PDK1 and 3 expression and a downstream decrease in PDH activity (193). The biologic relevance of these pathways was demonstrated by showing that inhibition of HIF-1 $\alpha$ decreased rates of PASMC proliferation. The contribution of a complex I activity defect to the glycolytic switch in PAH has also been shown by Raflkov et al. in PASMC from MCT rats (255). There are additional mechanisms of PDH inhibition and glycolytic shift in PAH PASMC. For example in MCT and Sugen-Hypoxia rats and in PAH patients, there is impaired function of the mitochondrial calcium uniporter complex (MCUC) function in PASMC. This decrease in the ability of mitochondria to uptake cytosolic calcium is caused by downregulation of the MCU subunit and upregulation of the inhibitory, 
mitochondrial calcium uptake protein 1 subunit (MCU1). The result, a fall in intramitochondrial calcium, reduces the activity of several calcium-dependent enzymes involved in glucose oxidation, including PDH, leading to uncoupled glycolysis (140). This MCUC abnormality in PAH is epigenetically regulated, by an increase in the expression of miR-25 and miR-138 (140). Downregulation of the MCU also promotes increased mitochondrial fission, as will be discussed subsequently (Figure 15).

PDH inhibition and aerobic glycolysis do not result exclusively from PDK activation or loss of MCUC function. Michelakis et al. found that sirtuin-3 (SIRT3) deficiency also reduces PDH activity and promotes glycolysis in SIRT3 knockout mice and IPAH patients (242). SIRT3 deficiency causes $\alpha$-ketoglutarate $(\alpha-K G)$-mediated HIF-1 $\alpha$ activation and increases the expression of PDK1, retinoid-interferon-induced mortality-19 (GRIM-19)-mediated increases in both signal transducer and activator of transcription 3 (STAT3) and glycogen synthase kinase 3 beta (GSK-3 $\beta$ ). These abnormalities culminate in activation of nuclear factor of activated T-cells, cytoplasmic 2 (NFATc2). A consequence of SIRT3-induced NFAT activation that relates to increased pulmonary vasculature tone in PAH is decreased Kv1.5 channel expression (242). More recently, Zhang et al. found that miR-449a-5p-mediated upregulation of Myc (also named p64), induced glycolysis in PASMC from both MCT and hypoxic rats (359). In both PAH patients and Sugen/Hypoxia rats, Kovacs et al. found that, as in PAEC, an increase in PFKFB3 promotes glycolysis in PASMC. This proglycolytic shift in turn results in extracellular signal-regulated kinase 1 and 2 (ERK1/2)-dependent activation of calpain-2, which promotes adverse pulmonary vascular remodeling (160). The production of mito-ROS in PASMC in PAH (as in HPV) is controversial. While our group shows a decrease in mito-ROS in PAH $(15,36)$, others have found 
the opposite $(255,359)$. The reason for these discordant findings is unknown, but it may depend on species/strain studied. We evaluated ROS in PAH PASMC derived from FHR and IPAH patients whilst ROS production in rats with MCT PAH and hypoxic rats with PH. In BMPR2 knockout mice, Rabinovitch et al. found hypoxic inhibition of ROS whereas in normoxia the mutation was associated with increased ROS, suggesting environmental and experimental considerations are critical determinants of the vector of change in mito-ROS in PAH (83). Nevertheless, regardless of the animal models including FHR, MCT rats, SIRT3 knockout mice, Sugen-hypoxia rats, hypoxic rats or patient group (IPAH or just PAH patients), all groups consistently report metabolic remodelling (a Warburg shift to aerobic glycolysis), an increase in $\Delta \psi_{m}(15,36,242,359)$ and increased mitochondrial fission $(15,33,140,359)$.

Two studies have examined FAO in PAH PASMC. The first study showed that mice lacking the gene for the metabolic enzyme malonyl-CoA decarboxylase (MCD) do not develop pulmonary hypertension during chronic hypoxia (301). The lack of MCD inhibits FAO and promotes glucose oxidation (an example of the Randle cycle) and thereby prevents the shift to uncoupled glycolysis. Other stigmata of adverse metabolic remodeling [i.e. downregulation of Kv1.5 and an increase in phosphorylated GSK-3 $\beta$ (p-GSK-3 $\beta$ ) and NFATc2] were also inhibited in hypoxic MCD mutant mice, demonstrating that the presence of functional MCD enhances FAO and exacerbates PAH. The therapeutic strategy of inhibiting FAO to benefit the pulmonary vasculature is consistent with prior findings showing that partially inhibiting FAO in $\mathrm{PAB}$ rats improved RV function and reduced RVH (96). Singh et al. found that hypoxia caused a metabolic shift in healthy human PASMC from glucose oxidation to FAO, due to an increase of FAS (289). These changes were associated with increases of Glut1, p-GSK-3 $\beta$, carnitine palmitoyltransferase 1 (CPT-1) and PDH 
inhibition (289), similar to findings in healthy human PAEC under hypoxic challenge (290). Moreover, increases in FAS caused apoptosis resistance (via decreases in Bax, caspase 3, annexin $\mathrm{V}$, and an increase in Bcl-2) and promoted autophagy (via decreases in p-mTOR, LC3BII/I ratio, and p62) (289). Finally, the increase of FAS is also associated with decreased mito-ROS and increased $\Delta \psi_{m}$. Bertero et al. cultured PASMC in stiff versus soft extracellular matrix (ECM) and found that stiff ECM activates YAP/TAZ, which increases several metabolic enzymes, including GLS1, LDHA and PC, leading to increased glutaminolysis and glycolysis (33). These findings in PASMC are the same as that in PAEC (33).

\section{Pulmonary artery adventitial fibroblasts}

A shift to aerobic glycolysis in PAH PAFib was first reported by Zhao et al. who showed that PAFib from IPAH patients exhibit increased expression of genes relevant to glycolysis, including Glut1, PDK1 and HK1 and manifested increased ${ }^{18} \mathrm{FDG}$ uptake, suggesting a shift to glycolysis (361). Later, Stenmark et al. found that PAFib from hypoxic neonatal calves and IPAH patients exhibit downregulation in complex I activity and NADH dehydrogenase [ubiquinone] iron-sulfur protein 4, mitochondrial (NDUFS4), leading to decreased PDH activity and increased glycolysis (246). These PAFibs also display upregulated expression in Glut1, HK2 and LDHA. As in other pulmonary vascular cells, the consequence of this metabolic remodeling in fibroblasts was apoptosis-resistance, hyperproliferation and a proinflammatory phenotype. The mechanism involved the NADH-sensitive transcriptional corepressor $\mathrm{C}$-terminal binding protein (CtBP1), which inhibits cyclin-dependent genes (p15 and p21), proapoptotic regulators phorbol-12myristate-13-acetate-induced protein 1 (PMAIP1, also known as NOXA), a p53 apoptosis effector related to PMP-22 (PERP), and the anti-inflammatory gene, heme oxygenase 1 (HMOX1) (173). 
Recently, Stenmark et al. found that the miR-124/PTBP1/PKM1/2 pathway that is activated in PAEC is similarly activated in PAFib (360). The two studies from Stenmark et al. reported increases in both mito-ROS and $\Delta \psi_{m}(246,360)$. These studies remind us there are several means of achieving a Warburg metabolic phenotype, including the DNMT-SOD2-HIF-1 $\alpha$-PDK-PDH pathways and the miR-124/PTBP1/PKM1/2 pathway, as summarized in a recent editorial (10).

\section{Pulmonary vascular pericytes}

Pericytes are perivascular supporting cells which assist maturation of blood vessels (22). Reduced pericyte-endothelial cell interaction is considered one of the underlying mechanisms causing rarefaction of the pulmonary vasculature. Yuan et al. performed a transcriptomic analysis of pericytes derived from lungs in controls and PAH patients and identified PDK4 as the top differentially regulated metabolic gene (356). This study showed that upregulation of PDK4 correlated with increased pericyte proliferation, decreased apoptosis and increased glycolysis. In addition, knockdown of PDK4 in PAH pericytes restored mitochondrial metabolism, reduced cell proliferation and improved pericyte-PAEC interactions in vitro, suggesting a mitochondrial basis for pericyte dysregulation to the small vessel loss and impaired angiogenesis observed in PAH. Interestingly PDK4 is also the predominant cardiac isoform that is upregulated in right ventricular hypertrophy, once gain highlight the shared benefits to the heart and lung that derives from restoring glucose oxidation in PAH.

In summary, all the cells in pulmonary circulation, including pericytes PAFib, PASMC and PAEC manifest altered mitochondrial metabolism, including increased uncoupled glycolysis, depressed glucose oxidation, altered FAO and/or increased glutaminolysis. In general, parallel benefits of 
enhancing glucose oxidation have been demonstrated in most pulmonary vascular cells and in the right ventricle. The similar benefit to RV myocytes and pulmonary vascular cells bodes well for mitochondrial-metabolic therapies, as they must be beneficial to the heart and lung in vivo.

\section{Mitochondrial fission and fusion}

Mitochondria are highly dynamic cellular organelles which continuously undergo fusion (joining individual mitochondrion together to become one) and fission (dividing one mitochondrion into multiple mitochondria). The consequences of fission and fusion must be viewed contextually. In certain circumstances they account for maintaining mitochondrial quality control via mitophagy (190), initiate cell death (310) or regulating metabolism and cell cycle progression (59) (Figure 7). In eukaryotes, the main proteins involved in mitochondrial fusion and fission dynamics are large GTPase proteins belonging to the dynamin family (280). The major mediator of mitochondrial fission is dynamin related protein 1 (Drp1), while fusion is mediated by GTPases mitofusin-1 (Mfn1), mitofusin-2 (Mfn2), and optic atrophy 1 (OPA1) (74, 221) (Figure 8). While inherited abnormalities of mitochondiral fission (Drp1; DNM1L mutations (337)) and fusion (MFN2 mutations) $(56,81,363)$ can cause disease, acquired abnormalities of fission (192) and fusion (273) are much more common and are noted to occur in PAH.

\section{Mitochondrial fusion mediators}

Mfn1 and Mfn2 are located on the outer mitochondrial membrane (OMM) and form homodimers $(56,126,159)$ or heterodimers (the most efficient method for fusion) $(126,141)$ to mediate OMM fusion. OPA1, another dynamin family GTPase, is located on the IMM and mediates its fusion $(188,294)$. Mitofusins hydrolyze guanosine triphosphate (GTP) to fuse adjacent mitochondria, 
allowing intermixing of the respective mitochondrial matrix contents (mtDNA, metabolites and proteins). Thus, fusion facilitates replacement of damaged mitochondrial components $(55,57$, 294). Deficient expression or function of OPA1, Mfn1 or Mfn2 leads to varying degrees of fragmentation of mitochondrial network and results in mitochondrial dysfunction $(57,144)$. Loss of Mfn1 leads to a greater degree of fragmentation than loss of Mfn2 (56). This can be explained by the fact that Mfn1 has a higher GTP-dependent membrane tethering activity than Mfn2 (144, 312). Reintroduction of Mfn1 or Mfn2 in Mfn2-knockout (KO) or Mfn1-KO, respectively, can restore mitochondrial fusion, suggesting an element of redundancy in the action of these fusion paralogs (56).

Mfn2 is also found on the surface of the endoplasmic reticulum (ER), where it regulates mitochondria-ER contact sites. These mitochondria associated membranes (MAMs), provide the physical basis for intercommunication between these organelles during $\mathrm{Ca}^{2+}$ signaling $(80,99)$ and are also critical for activation of the NACHT, LRR and PYD domains-containing protein 3 (NLRP3) inflammasome (213). Fusion is also important for controlling cellular homeostasis and cell survival. A recent study by Hoppins et al. suggests that soluble, monomeric Bax (an apoptosis regulator) selectively interacts with $\mathrm{Mfn} 2$ on the surface of mitochondria and, through conformational changes, enhances mitochondrial fusion. During apoptosis, the loss of soluble, cytosolic, Bax attenuates mitochondrial fusion (141).

Mfn1 and Mfn2 share identical functional domains. The conserved domains include an N-terminal GTP binding domain and heptad repeat coiled-coil region (HR1) and two closely spaced transmembrane (TM) segments, and a second coiled-coil domain (containing heptad repeat HR2) 
located on the C-terminal; both the GTPase and the two HR domains face the cytosol. In addition, Mfn2 possesses a Ras-binding domain at its N-terminal that is absent in Mfn1, suggesting specific roles of Mfn2 (60). Overexpression of Mfn2 by adenovirus-mediated gene transfer can inhibit cell proliferation and induce apoptosis of rat smooth muscle cells $(60,121)$. There remains some debate whether Mfn2 can regulate cell proliferation only by causing fusion (273) or through fusionindependent signaling mechanisms (58). The accepted mechanism of Mfns-mediated mitochondrial fusion is a HR2 trans model, i.e. the HR2 domain near the C-terminus of a Mfn interacts with another HR2 of Mfn on an adjacent mitochondrion. The OMM interaction of two opposing mitochondria is established via their HR2 domains in a dimeric antiparallel form, followed by GTP hydrolysis-induced OMM fusion (159).

The human dynamin-related protein OPA1 is anchored to the IMM via a single-span transmembrane domain which is close to the N-terminal and downstream of the mitochondrial matrix targeting sequence, and the majority of OPA1 faces the inter-membrane space (230). IMM fusion occurs after the OMM fusion. OPA1 not only mediates IMM fusion but also sequesters cytochrome $c$ within the mitochondria. OPA1 requires Mfn1 to promote mitochondrial fusion, but interestingly not Mfn2. Knockdown of $O P A 1$ with siRNA blocks mitochondrial fusion resulting in mitochondrial fragmentation, depolarization of mitochondrial membrane potential and altered mitochondrial IMM structure, which in aggregate, lead to cytochrome $c$ release, caspase activation, and apoptosis $(23,68,170,228)$.

The regulation of mitochondrial fusion protein expression occurs by several mechanisms. The protein levels of mitofusins are mainly regulated by the ubiquitin-proteasome system (70), and few 
post-translational modifications have been reported. Mfn1 can be phosphorylated by extracellularsignal-regulated kinase (ERK), a mitogen-activated protein (MAP) kinase cascade member, inhibiting mitochondrial fusion and inducing apoptosis (253). Under mitochondrial stress conditions, Mfn1 levels are regulated by mitochondrial E3 ubiquitin-protein ligase, membraneassociated ring finger (C3HC4) 5 (MARCH5), which mediates proteasomal degradation of acetylated Mfn1 (239). In response to glucose starvation, histone deacetylase HDAC6 interacts with Mfn1 leading to deacetylation and activation of Mfn1, promoting mitochondrial fusion (168), perhaps serving a compensatory mechanism to improve energy homeostasis and prevent oxidative damage. Mfn2 can also be regulated by the ubiquitin-proteasome system. In response to cellular stress, Mfn2 is phosphorylated by Jun N-terminal kinase (JNK), which leads to recruitment of the ubiquitin ligase (E3) Huwe1 to Mfn2, resulting in ubiquitin-mediated proteasomal degradation of Mfn2 and mitochondrial fragmentation (166). Additionally, PTEN-induced putative kinase (PINK1) phosphorylates Mfn2 in response to mitochondrial depolarization and promotes its Parkin-mediated ubiquitination and proteasomal degradation. This is required for quality control and elimination of dysfunctional mitochondria (61). Moreover, MITOL [also known as MARCH5, a mitochondrial ubiquitin ligase which belongs to the membrane-associated RING-CH E3 ubiquitin ligase (MARCH) family] ubiquitinates mitochondrial Mfn2, but not ER-associated Mfn2, and activates it for ER-mitochondria tethering (299).

Mfn2 is also regulated at the transcriptional level. Peroxisome proliferator-activated receptor $\gamma$ coactivator 1- $\alpha$ (PGC-1 $\alpha)$ is one of the transcriptional regulators of Mfn2 expression (273). OPA1 is regulated by alternative splicing and proteolysis. The differential splicing of OPA1 mRNA generates eight transcript variants (229). The long isoforms of OPA1 are generated from the eight 
splice isoforms by mitochondrial processing peptidase-mediated cleavage (293). In contrast, OPA1 also undergoes proteolytic cleavage at other sites, S1 and S2, downstream from the transmembrane domain. This generates short, transmembrane domain-free OPA1. Two metalloproteases, OMA1 and YME1L, cleave OPA1 at protease sites S1 and S2, respectively $(131,293)$. Loss of mitochondrial membrane potential triggers OPA1 cleavage at S1, but not S2, resulting in the inactivation of OPA1, promoting selective removal of defective mitochondria (mitophagy). This process improves the quality of the residual mitochondria (131).

\section{Mitochondrial fission mediators}

Mitochondrial constriction and scission (the last step of mitochondrial division resulting in two mitochondria) are executed by the GTPases dynamin-related protein 1 (Drp1) and dynamin 2 (Dnm2, Dyn2), respectively. In human cells, the average diameter of mitochondrial tubes is approximately $300 \mathrm{~nm}$ (161). Both Drp1 and Dyn2 oligomerize into rings that are much smaller than the diameter of mitochondrial tubes, suggesting a pre-Drp1 constriction step is needed to reduce the diameter of mitochondria. Voeltz et al. have reported that ER tubules wrap around mitochondria to drive pre-constriction and define the position of future mitochondrial division sites, where assembly of Drp1 will ultimately occur (104).

Drp1 lacks a membrane-anchoring domain and is primarily located in the cytosol. Activated Drp1 is recruited to the surface of mitochondria during organelle fission where it binds to several Drp1 receptor proteins on the OMM. On the OMM, activated Drp1 assembles into higher order oligomers and forms a contractile, ring-like, structure around the mitochondria at ERmitochondrial contact sites, leading to further narrowing of the mitochondria. The final scission of 
mitochondria has been proposed to be carried out by Dyn2 (104, 161, 231). Indeed, both Drp1and Dyn2-depleted cells showed similar hyper-fused mitochondrial morphology (167). Recently, Ryan et al. reported that Drp1 has both membrane constricting and severing abilities and is sufficient to perform mitochondrial and peroxisomal fission in the absence of Dyn proteins (153).

There are four OMM-anchored Drp1 receptors: fission protein 1 (Fis1), mitochondrial fission factor (Mff), mitochondrial dynamics protein of $49 \mathrm{kDa}$ (MiD49), formerly known as mitochondrial elongation factor 2 (MIEF2) and mitochondrial dynamics protein of $51 \mathrm{kDa}$ (MiD51), formerly known as mitochondrial elongation factor 1 (MIEF1) (182). Overexpression of Fis1 promotes mitochondrial fission, whereas inactivation of Fis1 induces an elongated mitochondrial morphology $(298,351)$. In the absence of Drp1 and Dyn2, human Fis1 (hFis1) can still mediate mitochondrial fission because of its ability to impair the function of fusion mediators. In the absence of Drp1 and Dyn2, hFis1 interacts with Mfn2 and OPA1, and impairs their GTPase activity, thereby inhibiting the fusion machinery and resulting in mitochondrial fragmentation (355). Knockdown of Mff causes the release of Drp1 from the OMM and results in mitochondrial fusion. Conversely, Mff overexpression enhances the recruitment of Drp1 to the OMM and increases mitochondrial fission. Mff-mediated mitochondrial fragmentation occurs independently of Fis1, suggesting that Fis1 is dispensable for Drp1 recruitment and mitochondrial division (233). Indeed, it appears that the crucial Drp1 binding partners likely varies both by cell type and pathophysiologic circumstance.

Both MiD49 and MiD51 are anchored in the OMM via their N-terminal (237). Unlike Fis1 and Mff, MiD49 and MiD51 do not also target to peroxisomes(237). MiDs recruit Drp1 specifically to 
mitochondria and act independently of Fis1 and Mff. Moreover, the Drp1 recruitment activity of Mff and Fis1 appears less efficient than that of MiD49 and MiD51 (236). The role of MiD49 and MiD51 in mitochondrial fission remains controversial. Palmer et al reported that overexpression of MiD49 and MiD51 elongates mitochondria; whereas our group finds the opposite (discussed below). Palmer's findings might be caused by sequestration of non-functional Drp1 to mitochondria, resulting in mitochondrial elongation; whereas overexpression of the MiDs at low levels forms discrete foci at the sites of mitochondrial constriction with Drp1 and Mff leading to fission (236). Both MiD49 and MiD51 contain a nucleotidyl transferase domain, however, ADP or GDP cofactors only bind to this domain in MiD51 (181). In the absence of nucleotide binding to MiD51, the recruited Drp1 cannot be activated for fission; whereas ADP binding promotes Drp1 assembly and mitochondrial fission (180). This suggests a link between metabolism and MiDmediated fission. Mff and MiDs cooperate in mediating mitochondrial fission and together are responsible for most Drp1 recruitment to mitochondria in PAH (27). However, the importance of specific binding partners may vary by cell type and differ amongst fissogenic stimuli. For example, while we have found a role for Fis1 in cardiomyocyte ischemia-reperfusion injury, as a mediator of fission and ROS formation (310), we found no such role in the accelerated mitotic fission which occurs in PAH PASMC (59). These binding partners may also have additive effects. For example, whilst knockout of either Mff, MiD49, or MiD51 causes partial inhibition of mitochondrial fission, triple knockout causes a complete inhibition of fission (232). This is consistent with our recent finding that in PASMC (normal or from PAH patients) MiD overexpression, whether due to transfection or disease, promotes fission whilst MiD knockdown reduces fission (59).

Regulation of fission mediators is mainly dependent on post-translational modifications and 
protein degradation. Drp1 undergoes several post-translational modifications including phosphorylation, SUMOylation, ubiquitination, and S-nitrosylation (283). Phosphorylation of Drp1 has been investigated extensively. Phosphorylation of Drp1 at serine 616 (S616) enhances mitochondrial fission, whereas cyclic AMP-dependent protein kinase A (PKA) phosphorylates Drp1 at serine 637 (S637) inhibits its GTPase activity and reduces mitochondrial fission, thereby protecting mitochondria from autophagosomal degradation $(52,75)$. During cell death, Drp1 S637 can be dephosphorylated by calcineurin, a calcium-dependent phosphatase, leading to Drp1 translocation to the mitochondria and increased mitochondrial fission $(48,75)$. Drp1 S616 can be phosphorylated by various kinases in response to different cellular physiological conditions. For example, in cells undergoing rapid mitosis, as in PAH and cancer, Drp1 S616 can be phosphorylated by cyclin B/CDK1, a key mitotic kinase (192). This phosphorylation of Drp1 that is coordinated with mitosis increases mitochondrial fission and is called mitotic fission. Mitotic fission is essential to ensure an equal distribution of mitochondria to the daughter cells $(192,283)$ In addition, S616 can also be phosphorylated by $\mathrm{Ca}^{2+} /$ calmodulin-dependent kinase II (CaMKII) during chronic $\beta$-adrenergic receptor ( $\beta$-AR) stimulation, resulting in mitochondrial permeability transition pore opening, mitochondrial damage, and cardiac dysfunction (344). Moreover, ERK1/2 phosphorylation of Drp1 S616 is requisite for Ras-induced cellular transformation and cell reprogramming $(250,281)$.

Drp1 is one of many fission/fusion proteins that are postranslationally regulated. In response to energy stress, Mff can be phosphorylated by AMP-activated protein kinase (AMPK), a cellular energy sensor. Phosphorylation of Mff enhances Drp1 recruitment and mitochondrial fission and improves the removal of damaged mitochondria (313). Likewise, cell stress regulates MiD49 
expression through MARCH5-triggered ubiquitination that leads to increased proteasomal degradation (343). This MARCH5-dependent MiD49 degradation is itself negatively regulated by Mff and Drp1 (62). Finally, it is clear the binding partners of Drp1 (and Drp1 itself) are epigenetically regulated. For example, in human and experimental PAH, expression of MiD49 and MiD51 is upregulated by a loss of miR-34a-3p (59). The expression of Drp1 itself is also epigenetically regulated by miR-30 in cardiomyocytes (172).

\section{Pathologic and therapeutic implications of dysregulated mitochondrial dynamics in PAH}

Increased mitochondrial fission in PAH is due in part to the increased expression and/or activity of Drp1 and its binding partners, MiD49 and MiD51 (Figure 9-10).

\section{Upregulation of Drp1 in PAH}

Increased Drp1-mediated mitochondrial fission was first identified in human PAH PASMC and shown to account for their fragmented mitochondrial network (192). Inhibition of Drp1 by a pharmacological agent, mdivi-1 (47), or molecular intervention (siDrp1) creates a fused mitochondrial network in PAH PASMC. This fusion in the face of a drive to proliferate, triggers cell cycle arrest at the G2/M phase, due to activation of CDK1/Cyclin B1 (192). Furthermore, in vivo experiments showed that inhibition of Drp1 reverses established experimental PAH, both as measured hemodynamically (reduced PA pressures) and histologically (reduced muscularization of small pulmonary arteries in MCT-PAH and chronic hypoxia PH) (192).

As previously discussed, HIF-1 $\alpha$ is activated in PAH PASMC compared to normal PASMC (8). To evaluate the pathologic relevance of this observation we exposed normal PASMC to cobalt. Cobalt activated HIF-1 $\alpha$ and recapitulated the fragmented mitochondrial network seen in PAH 
PASMC. This HIF-1 $\alpha$-induced mitochondrial fission was dependent on Drp1 and was reversed by mdivi-1. Exposing rats to cobalt chloride $\left(\mathrm{CoCl}_{2}\right), 2 \mathrm{mg} \mathrm{CoCl} 2$ i.p./day for four weeks induced mild pulmonary hypertension with adverse pulmonary vascular remodeling which was prevented by in vivo therapy with weekly injections of mdivil $(50 \mathrm{mg} / \mathrm{kg})$. Thus, HIF-1 $\alpha$-induced mitochondrial fission contributes to the development of PAH by accelerating cell cycle progression and Drp1 inhibition has therapeutic potential.

The role of fission in PAfib is less well understood. However, upregulated expression and increased activation of Drp1 does fragment the mitochondrial network in RVfib derived from the preclinical MCT-PAH model (311). The elevated Drp1 activity in MCT-RVfib is associated with its hyperproliferative, proglycolytic phenotype and with the increased collagen production, which ultimately increases RV stiffness and promotes RV failure (311). Inhibition of Drp1 by mdivi-1 or a competitive peptide, P110, which selectively blocks interaction between Drp1 and Fis1 (254), decreased mitochondrial fission, cell proliferation and collagen expression in MCT-RVfib. Thus, Drp1 is a conserved target amongst cells relevant to PAH in the cardiopulmonary unit. Drp1 may be a promising therapeutic target in the treatment of PAH. Of note, inhibition of Drp1 and/or Drp1Fis1 interaction are effective in treatment of other cardiac and pulmonary diseases which are characterized by increased mitochondrial fission, including lung cancer (259) and RV ischemiareperfusion injury (310). As with the metabolic inhibitors, which are beneficial in all pulmonary vascular cell types and the RV, it appears that inhibition of Drp1 is beneficial in PAfib, PASMC and is beneficial to the right ventricle, both during PAH and when there is ischemia-reperfusion injury. 


\section{Upregulation of Drp1 in bronchopulmonary dysplasia (BPD)}

BPD is a form of chronic lung disease that develops in premature infants who have been exposed to mechanical ventilation and hyperoxia (222). BPD is characterized by alveolar simplification (69) and confers an increased risk of pulmonary hypertension in adulthood (155). In a mouse model of BPD induced by exposing mouse pups to hyperoxia, Drp1 expression and activity were increased (decreased Drp1 phosphorylation at S637) and Mfn2 expression was decreased (307). However, the authors did not investigate the physiologic consequences of these changes in protein expression to mitochondrial morphology or hemodynamics in their model.

\section{Upregulation of MiD49 and MiD51 in PAH}

MiDs are ubiquitously increased in various human PAH tissues, including pulmonary arteries, PASMC, and BOEC, as well as in pulmonary arteries and PASMC from MCT-PAH and Sugen/hypoxia-PAH (59). MiD51 is also increased in both left heart and right heart from a rodent model of group $2 \mathrm{PH}$, the supra-coronary aortic banding model (341). Increased MiD expression contributes to mitochondrial hyperfragmentation and the hyperproliferative, apoptosis-resistant phenotype of PAH PASMC. Inhibition of MiDs by specific siRNAs restores normal mitochondrial morphology (Figure 11) and arrests cell cycle progression at the G1 phase. Conversely, introducing exogenous MiDs in normal PASMC is sufficient to increase mitochondrial fission and accelerate cell proliferation. Pathway analysis shows that MiDs accelerate cell proliferation through ERK1/2 and cyclin-dependent kinase 4-(CDK4)-dependent mechanisms (59). A genomic study of PAH patients with either isolated post-capillary pulmonary hypertension (Ipc-PH) or combined postcapillary and pre-capillary pulmonary hypertension $(\mathrm{Cpc}-\mathrm{PH})$ patients identified a SNP (exm1300952) in the gene encoding MiD49, (SMCR7), that is associated with higher risk of PAH 
The upstream regulation of MiD49 and MiD51 has also been elucidated. Using a miRNA microarray assay and bioinformatic analysis, Chen et al. identified miR-34a-3p as a regulatory miRNA of both MiD49 and MiD51 (59). This miR is downregulated in human PAH. Direct binding of miR-34a-3p to MiD 3'-UTR was confirmed by luciferase activity assay. Administration of a miRNA-34a-3p mimic or anti-miR-34a-3p recapitulated the effect of exogenous MiDs or siMiDs on mitochondrial morphology and cell proliferation in PASMC, respectively. These findings indicate that the miR-34a-3p-MiDs pathway is pathogenic in PAH. Moreover, circulatory levels of miR-34a-3p level are decreased in PAH patients in two distinct cohorts. These promising data suggest that miR-34a-3p may have value as a potential biomarker for PAH. Based on preclinical studies, the miR-34a-3p-MiD pathway may also be amenable to therapeutic intervention through administration of siMiDs or miR-34a-3p by airway nebulization.

\section{Pathologic and therapeutic implications of decreased mitochondrial fusion in PAH}

\section{Downregulation of Mfn2 in PAH}

Mfn2 was originally described as a hyperplasia suppressor gene (60). Decreased mitochondrial fusion also contributes to the excessive mitochondrial fragmentation in PAH. Mfn2 expression is downregulated in pulmonary vascular media and PASMC from PAH patients (273). Likewise, pulmonary vascular Mfn2 expression is decreased in rat PAH models (MCT-PAH and Sugen/Hypoxia PAH), indicating that decreased Mfn2-mediated mitochondrial fusion is a common pathway in the pathogenesis of both human and experimental PAH. Overexpression of Mfn2 in PAH PASMC increases mitochondrial fusion. This forced fusion inhibitss cell proliferation and induced apoptosis. Interestingly, augmenting Mfn2 in normal PASMC also 
inhibited cell proliferation and induced apoptosis. Therefore, Mfn2-mediated cell cycle progression is involved in both health and disease. The observation that cell cycle progression is inhibited and apoptosis enhanced by a fused mitochondrial network, whether caused by Drp1 inhibition or overexpression of Mfn2, indicates the importance of mitotic fission to the pathologic phenotype of PAH PASMC (i.e. mitochondria must be able to divide for the cell to divide).

Investigation of the basis for Mfn2 downregulation in PAH revealed downregulation of Mfn2's transcriptional coactivator, peroxisome proliferator-activated receptor $\gamma$ coactivator $1-\alpha$ (PGC-1 $\alpha$ ) as the likely cause. Supplement of Mfn2 by airway nebulization of Mfn2 adenoviral gene therapy improved exercise capacity, hemodynamics and pulmonary vascular remodeling in $\mathrm{Su} / \mathrm{Hx}-\mathrm{PAH}$ rats (273).

Mfn2 expression is also decreased in lungs of rats with hypoxia-induced pulmonary hypertension (HPH) (95). Incubation of normal PASMC in an hypoxic environment decreases Mfn2 expression and increases phosphorylated Akt (p-Akt) expression. As in PAH models, this study identified Mfn2 downregulation as being a critical promoter of PASMC hyperproliferation. Overexpression of Mfn2 or inhibition of Akt's regulator, phosphatidylinositol 3-kinases (PI3K), decreased p-Akt as well as cell proliferation. Thus, the PI3K/Akt pathway is critical to Mfn2-mediated inhibition of cell proliferation.

\section{Regulation of Mfn2 in PAH}

In addition to transcriptional regulation by $\mathrm{PGC}-1 \alpha, \mathrm{Mfn} 2$ is epigenetically regulated by various miRNAs, including miR-17. miR-17 belongs to the miR-17/92 cluster and directly targets $M f n 2$ 
by binding to the 3'-UTR of the Mfn2 gene. miR-17 expression is significantly increased in both PAH patients and rodent models of PAH $(46,184)$. miR-17 mediates PASMC proliferation and apoptosis in an Mfn2-dependent manner (184); however, the potential therapeutic effect of antimiR-17 in vivo has not been investigated in preclinical models of PAH.

\section{Upregulation of Mfn1 in hypoxic pulmonary vascular remodeling}

Sustained exposure to hypoxia activates HIF-1 $\alpha$ and leads to HPH, a condition characterized by medial hypertrophy of small intrapulmonary arteries and increased rates of PASMC proliferation (319). Mitochondrial morphological changes in pulmonary vascular cells HPH models include mitochondrial vacuole formation and fragmentation (185). However, immunoblots of the mitochondrial dynamic regulatory proteins showed increased expression of a pro-fusion protein, Mfn1 (185). Surprisingly, the increased expression of Mfn1 was found to be pro-proliferative in this study. This was confirmed by gain-of-function and loss-of-function in vitro experiments. siMfn1 arrests cell cycle progression in the $\mathrm{S}$ phase. MiR-125a is a direct upstream regulatory miRNA of Mfn1 in hypoxia-induced pulmonary vascular remodeling and miR-125a antagomir is a potential therapeutic agent. It is unclear if this paradoxical effect of Mfn1 in this study relates to the differences between Mfn1 and Mfn2 in terms of effects on proliferation, the use of chronic hypoxic model (representative of Group $3 \mathrm{PH}$ rather than Group $1 \mathrm{PH}$ ), the effects of miR-125a on targets in addition to Mfn1 or other technical factors. In most vascular beds, mitofusins are antiproliferative $(58,60,79,273,342)$, unlike the findings of Ma et al. (185).

Imbalanced mitochondrial fission/fusion causing excessive mitochondrial fragmentation is detrimental in PAH and BPD. This is due in part to the upregulation or abnormal activation of 
fission-related genes (Drp1, MiD49, MiD51) and/or downregulation of fusion-related genes (Mfn2). The fission/fusion imbalance contributes to the hyperproliferative phenotype of pulmonary vasculature in PAH and possibly HPH and in preclinical models can be therapeutically targeted with beneficial results.

\section{Transcriptomics reveals altered expression of mitochondrial metabolic genes in PAH}

The advent of high throughput technologies like microarrays have revolutionized discovery in a variety of complex diseases. Microarrays are platforms onto which hundreds, thousands or tens of thousands of oligonucleotide probes are immobilized, where each probe set is specific and complimentary to an individual gene (136). Through the hybridization of amplified and labelled transcript, a transcriptome-wide signature can be obtained for each sample in an experimental condition. Comparison of the signals, together with appropriate normalization and multiple test correction allows for a ratio that is considered to be a fold-change expression as a consequence of the treatment or experimental condition. Because each gene can be described using a controlled vocabulary of terms that places the gene into multiple and overlapping functional annotations, it is possible to identify key molecular functions that are 'enriched', and so gain insight into overall functions (248). In order to identify the functional annotation of regulated genes within the PAH lungs, we used the National Centre for Biotechnology Information (NCBI) repository called the Gene Expression Omnibus (GEO) to mine for microarray data that has been published from human lungs of PAH patients and controls. We identified a recent paper that used microarrays to profile lung tissue from 58 PAH patients (15:43 male:female, 30 white, 6 black, 7 Hispanic, 5 Asian) and 25 failed donors (18:7 male:female, 21 white, 4 black)(297). The PAH cohort in this study included idiopathic (32), hereditary (18), associated (5) and other (4) PAH subtypes. We remined the data 
(using default protocols in the ncbi tool, GEO2R) based on 25 failed lung donors, and lungs from 32 idiopathic PAH patients and identified 2,970 genes that are differentially regulated between PAH and Control (corrected p-value $<0.05$; Supplementary Table 1)(297). When we used DAVID to perform functional analysis on 'Official Gene Symbols' (2,297 annotated genes). We identified 137 significantly enriched functional groups (Benjamini corrected p-value $<0.05$; Supplementary Table 2). In this dataset, we find significantly enriched 'KEYWORDS' and 'GOTERMS' that correspond to mitochondrial/metabolic functions. Because each of these functional groups contain regulated genes that may appear in other functional groups, we combined all genes in a manner that excluded repetition. This analysis revealed 434-genes (Supplementary Table 3) that are differentially regulated in the lungs of $\mathrm{PAH}$ patients that are specifically involved in mitochondrial/metabolic functions. These findings corroborate what we know from the right ventricle transcriptome of an the MCT-APH rat. We recently used Next Generation Sequencing of RNA (RNAseq) to investigate differences between the transcriptome profile of the RV of the MCT-PAH RVF model compared to control rats. Because RNAseq incurs the problem of multiple testing, this careful phenotyping was critical in order to understand the molecular mechanisms that cause, or respond to RVF. Hemodynamic phenotyping demonstrated that the MCT treatment established a severe PAH and RVF (increased RVSP, increased mPAP, increased RVEDV, decreased CO, TAPSE and impaired ventricular-arterial coupling). Histological assessment of collagen, CD68 demonstrated that the MCT RV was both fibrotic, and in a state of increased inflammation. Importantly, we showed significant, functional, mitochondrial defects in the MCT RV, notably decreased PDH, Complex1 and Complex IV activity. We then extracted RNA from the MCT RV and prepared libraries for RNA sequencing (RNAseq). RNAseq is a method by which RNA from biological samples can be prepared into fragmented libraries so that the sequence of 
each fragment can be read. The large datasets that result from these experiments take the format of short (75-150 nucleotide) reads from one (single-end) or both (paired-end) ends of each fragment that has been sequenced. These reads need to be aligned back to the reference genome in order for transcript assembly and counting so that differentially regulated genes can be identified. In the MCT RV, we identified 2,546 transcripts that were significantly and differentially expressed (1,457 upregulated and 1,089 downregulated). To understand the overarching functions of the genes that were dysregulated in the MCT RV, we applied functional annotation analysis to the list of differentially regulated transcripts. This approach relies on each gene being annotated using a controlled vocabulary so that apparently disparate genes involved in similar functions can be identified and grouped. In the MCT RV, the most significantly enriched terms converged on functions or gene ontologies (GO) that included mitochondria/metabolic, fibrosis and inflammation. We then mined data that has been published by other groups that have used microarrays to model transcriptome level changes in the RV of a mouse model of chronic RV outflow tract obstruction(163) and the RV from human PAH patients who had mutations in the $B M P R 2$ gene (132). Of the 347 genes we identified as being differentially regulated in our MCT rat and involved in functions related to either mitochondria or metabolism, 92 genes were commonly regulated in the $\mathrm{MCT}$ rat $\mathrm{RV}$ (corrected $p<0.05 ; \mathrm{n}=6$ ), the pressure overload mouse $\mathrm{RV}$ (corrected $p<0.1 ; \mathrm{n}=2$ ) and the human BMPR2 RV (corrected $p<0.1 ; \mathrm{n}=2$ ). These genes reflect the fingerprint of RVF that translates to human pathology. Importantly, this fingerprint is defined primarily by mitochondrial and metabolic dysfunction.

Taken together, transcriptomic data shows an enrichment of terms that describe mitochondrial/metabolic function in the lungs of humans with PAH compared to failed donors. 
This is consistent with data generated by our group in the MCT-RV, and human PAH-RV, suggesting a shared pattern of transcriptomic dysregulation of mitochondrial metabolic pathways in both the RV and lung in PAH (Table 4). This parallel mitochondrial dysregulation across the cardiopulmonary unit bodes well for mitochondrial targeted therapies that would, by necessity, affect both the RV and pulmonary circulation.

Transcriptomics is a powerful tool that is capable of reading the entire transcriptome of a biological sample so that when samples from different biological conditions are compared, the differential regulation of individual transcripts can be resolved, and these transcripts can be placed into a broader understanding of cellular function so that underlying mechanisms that were not previously apparent could be elucidated. We have remined existing data (not our own) from the lung of PAH patients compared to controls and identified a large number of differentially regulated genes which when placed through a functional analysis pipeline, revealed significantly enriched terms that correspond to mitochondrial/metabolic functions. Given that our own recent RNAsequencing data from the hearts of an animal model (and subsequent comparison to human data), we argue there is clear evidence for a dysregulation of mitochondrial/metabolic genes in the cardiopulmonary unit in PAH.

\section{Epigenetic regulation of mitochondrial function in pulmonary vascular diseases}

Epigenetics refers to changes in gene function that are due to changes in gene expression without alterations in the gene sequence (340). Epigenetic modifications account for gene-environment interactions and occur by three major mechanisms: DNA methylation, post-translational modifications of histone tails and noncoding RNAs (e.g., microRNAs, miR). The involvement of 
these mechanisms in the pathogenesis of complex diseases, including PAH is increasingly recognized, reviewed in (157). While epigenetic mechanisms can affect a single gene, they more commonly effect multiple genes simultaneously, demonstrating not only the importance of these mechanisms in such diseases, but the difficulty in resolving the role of epigenetic modifications in the wider genomic, transcriptomic and molecular environment. An important consideration for PAH therapy is that epigenetic mechanisms are potentially reversible using inhibitors of DNA methylation or HDAC inhibitors (which are already in clinical use), or potentially by administration of miRs or anti-miRs. This opens new therapeutic approaches to PAH, although one which is conceptually different from the single target-specific approach currently used for pharmacologic therapies, such as endothelin antagonists or guanylate cyclase stimulators. Epigenetic interventions will likely activate and/or inhibit broad pathways and the therapeutic benefit or toxicity will require a broad molecular and biochemical toxicity assessment, in addition to the conventional measurement of primary endpoints, such as effects on pulmonary vascular hemodynamics, functional capacity, RV function and mortality.

\section{DNA methylation}

DNA methylation is an epigenetic mechanism in which methyl groups $\left(-\mathrm{CH}_{3}\right)$ are covalently transferred onto the cytosine residues at the C5 position. DNA methylation largely occurs in $\mathrm{CpG}$ islands, regions of the DNA near the promoter which are enriched in $\mathrm{CpG}$ dinucleotides. Studies have revealed $\sim 60-80 \%$ of $\mathrm{CpGs}$ within somatic cells are methylated in human genomes. The $\mathrm{CpG}$ islands are formed from the clusters of ummethylated CpGs (291). DNA methylation is mediated and maintained by DNA methyltransferases (DNMT) 1, 3a and 3b. DNA methylation represses gene expression by three mechanisms i) Direct prevention of binding of the transcriptional 
regulator by the methyl group; ii) Indirect inhibition of transcription by methylation-facilitated binding of methyl-CpG binding proteins, which prevents recruitment of transcription factors; iii) Recruitment of large protein complexes by methyl-CpG binding proteins that modify chromatin structure reducing DNA accessibility by transcription factors (150).

\section{DNA methylation of mitochondrial SOD2}

Superoxide dismutase 2 (SOD2) is the mitochondrion's main enzyme for conversion of toxic superoxide anion into more stable, diffusible, hydrogen peroxide. The SOD2 gene is located on chromosome number 6 in humans and chromosome 1 in rats (65). SOD2 is a tumor suppressor gene (314). Epigenetic silencing of the SOD2 gene by hypermethylation has been reported to enhance cell proliferation in multiple malignancies, including pancreatic cancer and myeloma (77, 138, 143). SOD2 expression is decreased in the lungs and PAs of PAH patients (38). We reproduced this finding in human PAH tissues and showed that SOD2 expression was decreased in the lungs and PAs of the FHR by $\sim 50 \%$, despite a normal SOD2 sequence (36). As in cancer, reduced SOD2 expression reflected epigenetic silencing of mitochondrial SOD2; however in FHR PASMC, loss of SOD2 decreased hydrogen peroxide production (36). This downregulation of SOD2 in PAH is mediated by transcriptional repression via methylation of two key CpG islands (one in the promoter and the other in an enhancer region). The decrease in SOD2 and loss of hydrogen peroxide mimics hypoxia, and as previously discussed this pseudohypoxia results in the hyperproliferative, apoptosis-resistant, population of PASMCs. The upregulation of DNMT1 and DNMT3b was only found in the lung, not in systemic arteries, a reminder of the tissue specificity of epigenetic mechanisms (15). We evaluated the therapeutic potential of this pathway and showed that suppressing the activity of methyltransferase, using a chemical inhibitor of DNMT that is 
clinically used in myelodysplastic syndromes, 5-Azacytidine, reversed the SOD2 hypermethylation and rescued SOD2 expression. Moreover, supplementation with a SOD mimetic decreased mPAP and thickness of the pulmonary arterial media in the FHR model (15).

\section{Histone modification}

The nucleosome is the basic unit of DNA compaction in eukaryote cells. It is organized as a histone octamer consisting of 2 copies of each core histones H2A; H2B; H3 and H4. The N-terminal of the histone called the "tail" can undergo post-transcriptional modification including addition or removal of small and large organic molecules (acetylation, methylation, phosphorylation, deamination, palmitoylation, ubiquitylation, SUMOylation, biotinylation, glycosylation and ADPribosylation). These modifications are essential for the control of gene expression through the modulation of chromatin compaction. Histone modification regulates several key functions including the DNA repair process, chromatin assembly, mitochondrial function, mitochondrial biogenesis, proliferation and apoptosis.

Histone acetylation was the first histone modification reported in the literature. It is a dynamic process controlled by the activity of the histone acetyl transferase (HAT) and the histone deacetylase (HDAC) which add and remove the acetyl group onto the lysine residues of histone protein, respectively. Functionally, addition of the negatively charged acetyl group neutralizes the positively charged lysine residues, loosening the interaction with histones, thereby increasing the accessibility of the DNA to the transcription machinery. Conversely, removing acetyl groups from histones tightens the chromatin and suppresses DNA transcription. In humans, the literature reports nine HATs subclassified in two groups, and 18 HDACs in four major subclasses (I; IIa; IIb; III 
and IV). Impaired expression of class I, IIa, IIb and III HDAC have been described in PAH, and were associated with broad aspects of disease pathogenesis; however, for this review, we only focused on the HDACs which are related to mitochondrial function.

The mitochondrial deacetylase SIRT3 is downregulated in human PAH PASMC and a MCT rat model of PAH (242). Artificial downregulation of SIRT3 expression inhibits apoptosis and activates several pulmonary hypertension-related transcription factors (e.g. HIF-1 $\alpha$, STAT3; NFATc2) and suppresses mitochondrial function [decreased PDH activity, decreased oxidative metabolism and Krebs' cycle metabolites as well as increased mitochondrial membrane potential $\left(\Delta \psi_{m}\right)$ ]. Consistent with this, SIRT3 knockout mice spontaneously develop PAH (242). Conversely, artificial upregulation of SIRT3 improves mitochondrial function of PAH PASMC and reverses experimental PAH. It is noteworthy that in the heart, SIRT3 can deacetylate the fusion mediator OPA1, increasing its GTPase activity and thereby preserving mitochondrial function, offering protection form oxidant injury (276). It is unknown if this is SIRT3-OPA mechanism is operant in pulmonary vascular cells in PAH.

Artificial inhibition of SIRT1 expression, another class III HDAC, also increases PASMC proliferation. After chronic hypoxia exposure, SIRT1 knockout mice manifest exacerbated vascular remodeling and develop PAH. Interestingly, chemical activation of SIRT1 decreased PASMC proliferation, which was associated with increased PGC-1 $\alpha$ expression and mitochondrial biogenesis (364).

Whilst SIRT1 and SIRT3 are the only HDACs directly associated with mitochondrial dysfunction 
in PAH; the literature suggests that other HDACs might also contribute to mitochondrial dysfunction observed in PAH. The expression of class I HDAC is increased in PAH patients, PAH rat models and chronic hypoxic calves $(174,362)$. Inhibiting class I HDACs, using valproic acid, decreased inflammation and improved RV function and reduced pulmonary vascular lesions in a rodent model of PAH. Recent reports show that HDAC1 colocalizes with mitochondria in cardiac myocytes in early cardiac reperfusion injury (135). Consistent with this, activation of class I HDACs, including HDAC1 is associated with mitochondrial dysfunction, suggesting that class I HDACs might also regulate mitochondrial function (179). However, this potential interaction between class I HDACs and mitochondrial function has not been investigated in PAH.

Boucherat et al. reported that HDAC6, a member of class IIB, is upregulated in human and preclinical models of PAH. Inhibition of HDAC6 using Tubastatin promotes mitochondrial depolarization and mitochondria-mediated cell death, and can reverse experimental PAH (37). Independent studies show that HDAC6 inhibition by Tubastatin induces mitochondrial fusion in striatal neurons (120). However, this potential effect of HDAC6 inhibition on mitochondrial dynamics has not been reported in PAH.

Acetylated histone marks are recognized or "read" by a protein family called bromodomain and extra-terminal domain (BET). Among these "readers", BRD4 is upregulated in PAH and associated with the development of the disease (203). Reduction of BRD4 activity using chemical inhibitors decreased mitochondrial membrane potential $\left(\Delta \psi_{m}\right)$, and improved mitochondrial function in human PAH PASMC. Consistent with this observation, inhibition of BRD4 reversed PAH in rat model of the disease. Based on this work, BRD4 has been proposed as a therapeutic 
target in human PAH and a BRD4 inhibitor is now being studied in a clinical trial (NCT03655704).

Methylation is another post-transcriptional modification of the histones implicated in the epigenetic control of gene expression. It results from the equilibrium of the activity of histone methyltransferase [HMT or protein lysine methyltransferase (PKMT)] and histone demethylase (the LSD demethylases and the JMJC demethylases). Histone methylation is less studied than histone acetylation and the contribution of histone methylation/demethylation to mitochondrial dysfunction in PAH remains relatively unknown. However, increased expression of the HMT, enhancer of zeste homologue 2 (EZH2), is observed in PAH PASMC and preclinical models of PAH $(4,284)$. EZH2 is a histone-lysine N-methyltransferase enzyme (EC 2.1.1.43) encoded by the EZH2 gene, that participates in histone methylation and, ultimately, mediates transcriptional repression._Increased EZH2 expression was associated with the pro-proliferative, and antiapoptotic phenotype of human PAH PASMCs (4). Interestingly, increased EZH2 expression is associated with the Warburg effect in cancer cells (238). Artificial inhibition of EZH2 improves mitochondrial function (reverting cells from glycolytic metabolism to oxidative metabolism) and decreases expression of HIF-1 $\alpha$. Nevertheless, the beneficial effect of EZH2 inhibition on mitochondrial function has not been investigated in PAH.

\section{MicroRNA}

MicroRNAs (miRNAs) are small non-coding RNA molecules consisting of $\sim 21-25$ nucleotides $(100,169)$. They are encoded by intronic DNA and bind to the 3'-untranslated region (3'-UTR) of target mRNAs causing mRNA decay or impairing mRNA translation. A single miRNA can regulate the expression of an array of genes and conversely multiple miRNAs can regulate the expression of a particular gene $(100,195)$. In general, the greater the expression of an miR, the lower the expression of its target mRNAs and their related proteins. Dysregulation of miRNAs has 
been shown to alter mitochondrial metabolic pathways that are critical for creating the pseudoneoplastic phenotype of PAH (Table 3). Caruso et al. demonstrated that downregulation of miR-124 contributes to Warburg metabolism and the hyperproliferative phenotype in circulating BOECs isolated from PAH patients. miR124 does this by upregulating PTBP1, which controls the expression of PK isoforms PKM1 and PKM2 (45), as previously discussed. A similar study in PA fibroblasts confirmed that downregulation of miR-124 increased the ratio of PKM1/PKM2 via the dysregulation of PTBP1, resulting in increased uncoupled glycolysis (Figure 12) (360). Furthermore, modulation of dysregulated miRNAs in PAH using miR mimics or antagomirs, can regress or recapitulate PAH in preclinical models $(59,140,266)$. Although preclinical studies have identified promising outcomes supporting the therapeutic potential of mitochondrial targeted miR mimics (or their antagomers), they also raise concerns about potential, harmful off-target effects. These concerns can be addressed by robust preclinical and clinical toxicology and transcriptomic surveillance. Since extracellular miRNAs are resistant to RNase degradation they are stable and may serve as ideal biomarkers to aid in diagnosis and predict prognosis in PAH patients (262, 279).

In summary, epigenetic mechanisms allow rapid modification of gene expression in response to physiological and pathological stress. Thus, it not surprising that several epigenetic dysfunction including DNA methylation, histone modification, and miRNA expression occur in the pulmonary vasculature in PAH. Several epigenetic mechanisms related to Sirt1, Sirt3, HDACs, DNMT and several miRs are associated with mitochondrial dysfunction in PAH. Investigation of epigenetic mechanism of mitochondrial dysfunction will likely identify new biomarkers and suggest new therapeutic approaches. Indeed several drugs that modify epigenetic targets are already in clinical trials in $\mathrm{PAH}$. 


\section{Transcription factors}

Transcription factors are proteins that bind to the specific sequence of DNA and promote transcription of genes, yielding messenger RNA. In PAH, several pathological stimuli including oxidative stress and hypoxia (or pseudohypoxia) can modulate complex biological pathways which, in turn, can alter the expression and/or function of various transcription factors. Here we focus on transcription factors that are known to affect mitochondrial biology in pulmonary hypertension.

\section{HIF- $1 \alpha$ and the pulmonary vasculature}

HIF-1 $\alpha$ expression and activity increase inversely with $\mathrm{PO}_{2}$ (Figure 13). HIF is a heterodimeric protein with $\alpha$ and $\beta$ isoforms. The $\beta$-subunit is constitutively expressed whilst $\alpha$-subunit expression increases logarithmically as $\mathrm{PO}_{2}$ falls. Both HIF-1 subunits contain basic helix-loophelix motifs that bind DNA, causing subunit dimerization $(53,322,346)$. HIF activity is finely regulated by the availability of $\mathrm{O}_{2}$, which regulates several enzymes that control HIF-1 $\alpha$ expression. In normoxia, hydroxylation of HIF-1 $\alpha$ is mediated by prolyl hydroxylase domain proteins (PHDs) $(91,145,147)$. PHD's oxygen-dependent hydroxylation marks HIF-1 $\alpha$ for ubiquitination by von Hippel-Lindau protein (pVHL), a tumor suppressor and E3 ubiquitin protein ligase (146). The ubiquitination of HIF-1 $\alpha$ requires hydroxylation at proline-402 and proline-564

(145, 147, 194, 354). Hypoxia decreases PHD activity thereby decreasing HIF-1 $\alpha$ proline hydroxylation, resulting in the stabilization of HIF- $1 \alpha$. Another HIF-1 $\alpha$ regulator, factor inhibiting HIF-1 $\alpha$ (FIH-1), is also inhibited by hypoxia. FIH-1 hydroxylates HIF-1 $\alpha$ at an asparagine residue within its C-terminal transactivation domain. This hydroxylation blocks the binding between HIF$1 \alpha$ and the transcriptional co-activators CBP and p300 (146). Hypoxic-inhibition of FIH-1 
contributes to activation of HIF-1 $1 \alpha$ target genes (Figure 13). Through these mechanisms, hypoxia stabilizes the expression of HIF-1 $\alpha$ and its DNA-binding activity in PASMC and endothelial cells (353). Once in the nucleus, HIF-1 $\alpha$ dimerizes with HIF-1 $\beta$ which allows DNA binding to the promoter region of genes that have a conserved hypoxia response element (HRE). Manalo et al. showed that overexpression of a constitutively active HIF-1 $\alpha$ led to increased transcription of 245 genes whilst expression of 325 genes decreased (189). They concluded that $\sim 5 \%$ of genes expressed in endothelial cells are regulated by HIF-1 $\alpha$.

\section{Normoxic activation of HIF-1 $\boldsymbol{\alpha}$ in pulmonary hypertension}

Pseudohypoxic HIF-1 $\alpha$ activation is seen both in PAH (Figure 13) and cancer and reflects abnormalities in the mitochondria-based mechanism of oxygen-sensing (36), as previously discussed. In PAH, the pseudohypoxic environment is created by a variety of factors, including the previously discussed epigenetic downregulation of SOD2. Low SOD2 expression lowers the production of its product, the redox signaling molecule $\mathrm{H}_{2} \mathrm{O}_{2}$, thereby creating a pseudohypoxic state. As in true hypoxia, pseudohypoxic activation of HIF-1 $\alpha$ upregulates the expression of a variety of genes, including PDK, resulting in the inhibition of PDH. PDH inhibition reinforces the low ROS state by inhibiting the mitochondrial ETC, as metabolism switches to a reliance on uncoupled glycolysis in the cytosol. HIF-1 $\alpha$ activation ultimately reduces the expression of various ion channels, including Kv1.5, leading to depolarized, calcium-overloaded PASMC.

Fijalkowska et al. demonstrated that PAEC from IPAH patients have elevated HIF-1 $\alpha$ expression under both normoxic and hypoxic conditions. They further demonstrated increased expression of carbonic anhydrase IX, a transcriptional target of HIF-1 $\alpha$ in the pulmonary arteries of PAH patients (98). One mechanism by which HIF-1 $\alpha$ expression increases, in addition to epigenetic SOD2 
dysregulation, is via increased activity of glucose-6-phosphate dehydrogenase (G6PD). This mechanism was observed in $\mathrm{CD} 133^{+}$progenitor cells, which are capable of differentiating into hematopoietic, endothelial, smooth muscle, and neuronal cell types. G6PD is the rate-limiting enzyme in the pentose phosphate pathway and has been found to contribute to the proliferation of PASMC in hypoxic rats by directing the cells to synthesize fewer contractile proteins (myocardin and $\operatorname{SM} 22 \alpha$ ), and increase the expression of the proliferative proteins, cyclin A and phosphohistone H3 (63). Activation of HIF-1 $\alpha$ not only changes metabolism (increasing glycolysis and inhibiting glucose oxidation), it also induces mitochondrial fission by promoting cyclin B1/CDK1dependent Drp1 phosphorylation at S616 (192).

\section{Signal transducer and activator of transcription 3 (STAT3)}

STAT3, a transcription factor, encoded by the STAT3 gene, is activated in PAH and also modifies mitochondrial function. STAT3 activation is mediated by phosphorylation at its tyrosine 705 residue (PY705) in response to cytokines such as IL6, growth factors such as endothelin 1 (ET1) and angiotensin II (AngII) $(28,78)$ and PDGF. STAT3 activation is followed by nuclear translocation, dimerization and DNA binding (40). Involvement of STAT3 and its downstream targets is well-documented in PAH. Piml (provirus integration site for Moloney murine leukemia virus), a proto-oncogene, is a key downstream signal of STAT3 activation (234). Paulin et al. showed that increased Pim1 expression in PAH PASMC correlated with the severity of PAH. Furthermore, heterologous overexpression of Pim1 induced PASMCs proliferation by inactivating the pro-apoptotic protein, Bcl-2 associated agonist of cell death (Bad), resulting in hyperpolarization of mitochondria and also by increasing NFATc2 activity (241). STAT3mediated activation of Krüppel-like Factor 5 (KLF5) in human PAH accounts, in part, for the hyperproliferative phenotype in PAH PASMC, including hyperproliferation, mitochondrial 
hyperpolarization, increased survivin expression and resistance to apoptosis (73). Localization of STAT3 in mitochondria reveals a new role of STAT3 in regulating the ETC. Silencing of STAT3 reduced complex I and complex II activity. Mitochondrial Stat3 functions as a modulator of mitochondrial respiration rather than as a transcription factor (289). In CD4 cells activated with IL6, mitochondrial STAT3 helps maintain calcium homeostasis, which is dysregulated in PAH (347).

$P P A R \gamma$ and $P G C-1 \alpha$

Peroxisome proliferator-activated receptor gamma (PPAR $\gamma$ ), a member of a family of nuclear receptors/ligand-activated transcription factors, regulates cell metabolism, cell proliferation, and inflammation (315). PPAR $\gamma$ is downregulated in PAH and HPH tissues, including endothelial cells (6) and PASMC (183). Decreased PPAR $\gamma$ induces cell proliferation, in part through activation of the ERK 1/2-NF-kB-Nox4 pathway (183). Rosiglitazone, a thiazolidinedione insulin-sensitizer and PPAR $\gamma$ agonist (263), attenuates pulmonary artery remodeling in $\mathrm{HPH}(76,156,224)$ and reverses vascular remodeling and RV failure in the $\mathrm{Su} / \mathrm{Hx}-\mathrm{PAH}$ model (171).

PGC-1 $\alpha$ is a master regulator of mitochondrial biogenesis (175) and transcriptional coactivator of Mfn2 (273). PGC-1 $\alpha$ is downregulated in human and experimental PAH, as reviewed in the Mitochondrial Dynamics section above. Ye et al. confirmed our findings of downregulated PGC$1 \alpha$ in PAH PASMC (273), noting decreased PGC-1 $\alpha$ expression in normal human PAEC exposed to hypoxia (348). In their study hypoxia also reduced mitochondrial membrane potential, ATP production and increased oxidative stress, in a manner that was reversed by metformin. PAECS in rats exposed to four weeks of chronic hypoxia had decreased PGC-1 $\alpha$ levels and their 
mitochondrial ultrastructure was abnormal, evident as disrupted cristae.

Yeligar et al. demonstrated that PGC-1 $\alpha$ 's partner, PPAR $\gamma$, is also downregulated in PAH. Downregulation of PPAR $\gamma$ (like downregulation of PGC-1 $\alpha$ ) disrupts mitochondrial dynamics and dysregulates mitochondrial function (350). Exposure of normal PASMC to hypoxia or knockdown of PPAR $\gamma$ fragments mitochondria (by downregulating Mfn2) and also decreases oxygen consumption rates. Loss of PPAR $\gamma$ also decreases expression of PGC-1 $\alpha$ in human PASMC and lung tissue from mice exposed to hypoxia. These results extend our prior findings of PGC-1 $\alpha$ induced downregulation of Mfn2 (273) and suggest a role for a disordered PPAR $\gamma$-PGC-1 $\alpha-M f n 2$ pathway in the acquired mitochondriopathy of PAH.

In summary transcription factors plays an important role in the pathobiology of PAH. Activation of HIF-1 $\alpha$ under normoxic condition upregulates an array of genes which are critical to the pathogenesis of PAH. Activation of HIF-1 $\alpha$ has been shown to alter metabolism leading to uncoupled aerobic glycolysis and induces mitochondrial fission, ultimately contributing to increased cell proliferation, one of the hallmarks of PAH. Similarly, STAT3 activation in PAH by phosphorylation at PY705 upregulates its downstream gene targets, including Pim1, KLF5. These in turn contribute to PASMC proliferation. Furthermore, STAT3 has been shown to localize in mitochondria, where it acts as a modulator of mitochondrial respiration. Downregulation of PPAR $\gamma$ contributes to the proliferation of endothelial cells and PASMC in PAH by stimulating the ERK 1/2-NF- $\kappa$ B-Nox 4 pathway. On the other hand decreased expression of PGC-1 $\alpha$ in PAH, leads to increased mitochondrial fission and PASMC proliferation via downregulation of Mfn2.

\section{Mitochondrial biogenesis and mitophagy}


In order to maintain an optimally functional network, constant mitochondrial turnover is required to discard dysfunctional and damaged mitochondria. The dysfunctional or defective mitochondria are selectively removed through a process known as mitophagy (25). New mitochondria are also provided as needed through the PGC-1 $\alpha$-dependent process of mitochondrial biogenesis (127). PGC-1 $\alpha$ promotes mitochondrial biogenesis by activating nuclear respiratory factor 1 (NRF1) and TFAM. TFAM promotes transcription of mitochondrial DNA (mtDNA, Figure 14) (318). Mitochondrial biogenesis is decreased in PAH patients and in experimental PAH $(111,348)$. There are many mediators affecting mitochondrial biogenesis in PH patients. Afolayan et al. reported reduced expression of PGC-1 $\alpha$, ETC subunits, and mtDNA copy number in an ovine model of persistent pulmonary hypertension of the newborn (PPHN). In another study, mice lacking endothelial BMPR2 developed PH and exhibited inhibition of mitochondrial biogenesis, as well as an increase in mitochondrial ROS production and reduction in mitochondrial membrane potential, when challenged with hypoxia (83). Enache et al. conducted time course studies of mitochondrial biogenesis factors and respiration in the RV, gastrocnemius, and left ventricle in MCT-PAH rats. They demonstrated an early decrease in expression of mitochondrial biogenesis genes in the skeletal muscle, followed by similar decreases in RV myocytes and gastrocnemius muscle. These changes were not seen in the left ventricle (90).

Mitophagy involves the coordinated activation of PTEN-induced kinase 1 (PINK1) and the E3 ubiquitin-protein ligase, Parkin. In dysfunctional or damaged mitochondria, PTEN is directed to the OMM and its proteolytic cleavage by mitochondrial processing peptidase (MPP) and presenillin-associated rhomboid-like (PARL) proteases is inhibited. PINK1 recruits Parkin which in turn polyubiquitinates several OMM proteins such as voltage-dependent-anion- 
selective channel 1 (VDAC1), Mfn1 and Mfn2. The polyubiquitinated proteins are then recognized by adaptor proteins such as p62, optineurin (OPTN) and NDP52, followed by the recognition by light chain 3 (LC3) and formation of autophagosome (109). Mitophagy can be induced by oxidative stress $(2,109)$. Dysregulated mitophagy promotes pulmonary hypertension. For example, in mice exposed to intermittent hypoxia, loss of endothelial cell UCP2 increases mitophagy, suppresses mitochondrial biogenesis and increases PAEC apoptosis (128).

\section{Calcium homeostasis}

Increase in cytosolic calcium concentration, $\left[\mathrm{Ca}^{2+}\right]_{\text {cyto }}$, drives pulmonary vasoconstriction and PASMC proliferation $(164,285,323)$. Elevation of $\left[\mathrm{Ca}^{2+}\right]_{\mathrm{cyto}}$ is multifactorial, resulting from increased calcium influx through the L-type calcium channels, activation of transient receptor potential channels (TRPCs) and activation of store-operated calcium channels $(223,249,358)$. In contrast, intramitochondrial calcium, $\left(\left[\mathrm{Ca}^{2+}\right]_{\text {mito }}\right)$ is separately regulated and serves different purposes (regulation of metabolism and buffering of cytosolic calcium)]. Physiologic levels of $\left[\mathrm{Ca}^{2+}\right]_{\text {mito }}$ are required to maintain activity of the three mitochondrial dehydrogenases, which is achieved either by activating oxoglutarate dehydrogenase and isocitrate, or by activating PDH phosphatase which in turn activates PDH. Increases in $\left[\mathrm{Ca}^{2+}\right]_{\text {mito }}$ are associated with inhibition of cell proliferation and increased apoptosis. Consistent with this, Teshima et al. showed that overexpressing mitochondrial uncoupling protein-2 (UCP2) in cardiomyocytes inhibits calcium influx into mitochondria and decreases ROS production (308). In another study, a metabolic switch to glycolysis was observed due to the impairment in calcium-sensitive PDH in SMCs lacking UCP2. Loss of UCP2 inhibited calcium flux from the ER to the mitochondria (84). Sutendra et al. demonstrated that hypoxia upregulates Nogo-B, a regulator of ER structure, in PAH PASMCs through activation of the ER stress-sensitive transcription factor, ATF6. They further 
demonstrated that induction of Nogo-B decreased ER-to-mitochondria phospholipid and calcium transfer by increasing the distance between the ER and mitochondria. Furthermore, mice lacking Nogo-B are protected from hypoxia-induced PAH (303).

Although calcium does enter mitochondria from the ER and UCP2 modulates mitochondrial calcium uptake, the predominant route for calcium entry is via a multiprotein channel complex, the MCUC, located in the IMM (103). The function of MCUC is crucial in maintaining the cytosolic and mitochondrial calcium balance and the activation of mitochondrial calciumdependent dehydrogenases (31). Two component proteins of the MCUC function as the poreforming subunits, MCU and the essential MCU regulator (EMRE). Lower expression and decreased function of MCU in both PAH and cancer $(140,191)$ contributes to decreased $\left[\mathrm{Ca}^{2+}\right]_{\text {mito }}$ and increased cytosolic calcium concentration, which result in PDH inhibition and fission, respectively (82). Loss of MCUC function promotes cell proliferation and resistance to apoptosis in human and experimental PAH PASMC (140). The cause of changes in MCU expression are largely epigenetic, due to increased expression of the regulatory microRNAs, miR-25 and miR$138(140,321)$ (Figure 15). In contrast, restoring MCU expression, by airway nebulization of antimiRNA 25 or -138, regresses experimental PAH (140) (Figure 15).

\section{Evaluation of disordered metabolism of pulmonary vascular diseases}

Advanced imaging modalities have evolved to provide non-invasive quantitative measurements of glycolytic abnormalities in the lung and RV in PAH (317). Current imaging techniques employ EKG-gating and respiratory gating to improve image structure and function correlation in the pulmonary vasculature and RV in preclinical models of PAH and in patients with $\mathrm{PAH}(3,5,24$, $67,96,162,361)$. Here, we briefly discuss the development of some mitochondrial-targeted 
molecular imaging probes for SPECT and PET techniques, which are used to evaluate the changes in cardiac metabolic activity, viability, blood flow and perfusion in preclinical and clinical studies.

\section{Glucose and fatty acid metabolic imaging}

The mitochondrial-targeted molecular imaging probes have been widely studied and developed to investigate the mitochondrial function of myocardium in cellular processes ranging from metabolism and apoptosis to ventricular remodeling. Our lab and others are pursuing the goal of using preclinical imaging platforms to measure mitochondrial-metabolic function in experimental PAH. This research has first advanced in the study of the RV in PAH, an organ affected by ischemia, due to reduced right coronary artery perfusion pressure and capillary rarefaction (271). Under ischemic conditions, the myocardium shifts from oxidative phosphorylation to reliance on glycolysis and glutaminolysis (244). There is also increasing evidence for a pathologic metabolic shift to uncoupled glycolysis in the absence of ischemia in both the lungs and heart $(42,113,124$, 193, 302, 345). Increased glucose flux is required to maintain ATP homeostasis when myocytes use the lower energy yielding glycolytic pathways. Molecular imaging can measure the related increase in glucose flux that is required to maintain energy homeostasis in vivo, using $2-\left[{ }^{18} \mathrm{~F}\right]-$ Fluoro-2-deoxy-D-glucose (FDG) as a PET radiotracer (54). An FDG-PET study performed by Xu et al. showed a threefold increase in glycolytic rates in the lungs of patients with compared to healthy controls, suggesting that glycolytic glucose metabolism is the primary energy source of idiopathic PAH cells (345). This study showed the robust potential of using glucose uptake as a novel imaging modality for diagnosis and evaluation of treatment response in PAH patients (345); but, did not elucidate whether FDG-PET could detect mild PAH. Their findings also supported the substantial alterations in cellular bioenergetics of IPAH endothelial cells, linking the human 
disease to avian and rodent forms of PAH (108).

Lung FDG-PET was used to monitor PAH progression and assessment of therapeutic regression by Marsboom et al., using the MCT-PAH model(193). Seven Sprague-Dawley rats were longitudinally imaged before MCT injection and weekly after injection, utilizing both pulsed-wave Doppler echocardiography and FDG-PET techniques (see Figure 16A-E). The arrow at the 3-week time point (Figure 16A-B) shows the systolic notching of the PA Doppler envelope developed after three weeks in MCT-PAH rats, a well-established indicator of severe PH (316). Serial lung FDG-PET scans revealed progressively increasing FDG uptake in the lungs of MCT animals, within two weeks of MCT injection, when PAH was still mild (Figure 16C-D). Thus, there is evidence that a metabolic shift in lung metabolism occurs early, simultaneously with onset of PAH (beginning between 1 and 2 week after MCT injection). Figure 16E shows that there is a significant correlation between FDG uptake and PAAT measurements $\left(\mathrm{r}^{2}=0.3, \mathrm{p}<0.001\right)$. In this study, FDG uptakes were normalized by dichloroacetate or imatinib therapy. To identify the origin of the increased pulmonary FDG uptake, laser capture microdissection of airway versus vascular tissue was used. Oxygen consumption and metabolism were measured in PASMC cultures. The expression of Glut1, which mediates FDG uptake, was upregulated in both the endothelium and PASMCs, but not in airway cells or macrophages, suggesting the metabolic shift is specific to the vasculature. PASMCs from MCT rats were hyperproliferative and displayed normoxic activation of HIF-1 $\alpha$ and Glut1 upregulation in proliferating vascular cells, accounting for increased lung FDG-PET uptake. This study showed the high sensitivity of FDG-PET to mild PAH and its capacity for monitoring therapeutic changes in the vasculature (193).

In another comprehensive study in patients with idiopathic $\mathrm{PAH}$, Bokhari et al. revealed that dual 
isotope PET scanning with both ${ }^{13} \mathrm{~N}$-ammonia $\left(\mathrm{NH}_{3}\right)$ and FDG is a feasible means for quantifying RV blood flow and metabolism in idiopathic PAH patients (34). The majority of the patients in the study were treated with a standard regimen for PAH, including endothelin receptor antagonists, prostacyclin analogs, phosphodiesterase inhibitors, diuretics, digoxin, and spironolactone (34). All patients underwent cardiac MRI imaging, for measuring the end-diastolic and end-systolic volumes and wall thicknesses. ${ }^{13} \mathrm{~N}-\mathrm{NH}_{3}$ PET resting perfusion imaging allowed quantitative analysis of myocardial blood flow (MBF) in each region of interest and globally for the RV and LV, while FDG-PET was used to quantify RV myocardial glucose uptake (MGU). The increased rate of glucose utilization in the RV, which is associated with disturbed cardiomyocyte $\mathrm{Ca}^{2+}$ homeostasis and an increase in cellular oxidative stress, indicated early RV metabolic functional impairment (140). MBF and MGU, and the ratio of RV/LV MGU were correlated to clinical parameters such as PA systolic pressure $(\mathrm{r}=0.75, \mathrm{p}=0.0085)$ and mean PA pressure $(\mathrm{r}=0.87$, $\mathrm{p}=0.001$ ). This study also showed a tendency toward a negative correlation between RV/LV MGU and maximum oxygen consumption $(\mathrm{r}=-0.59, \mathrm{p}=0.05)$. They thus suggest PET imaging might serve as a novel early biomarker that could evaluate therapies and monitor disease progression (34).

Saygin et al. used FDG-PET to evaluate both lung metabolism and right heart metabolism and function in $\mathrm{PH}$ and investigated the correlation between quantitative PET measurements with echocardiographic results and other traditional assessments, like N-terminal pro B-type peptide (NT-proBNP) levels and 6-minute walking distance test (277). Average lung SUVs were Isignificantly higher in PAH vs healthy controls $(0.5 \pm 0.15$ vs. $0.37 \pm 0.09 \mathrm{~g} / \mathrm{mL}, \mathrm{p}=0.01)$, confirming prior studies (124). However, lung SUV did not correlate with NT-proBNP levels, or 
echocardiographic measurements (269). This study showed that a single gated FDG-PET, in the fasting state, is potentially more accurate than 2D-echocardiography for measuring both heart metabolic/functional parameters, and can also achieve metabolic evaluation of the lungs (277).

The healthy adult heart relies primarily on FAO, rather than glucose metabolism, as a source of energy production (296). Most imaging studies in PAH have evaluated glucose uptake in RV function in PAH, while only few studies assessed fatty acid metabolism in these patients. In 1998, Nagaya et al. used 15-(p-iodophenyl)-3-(R,S)-methylpentadecanoic acid (BMIPP) radiolabeled with ${ }^{123}$ Iodine and ${ }^{99 \mathrm{~m}} \mathrm{Tc}$-sestamibi (MIBI) to perform SPECT imaging for calculating the RV-to$\mathrm{LV}$ tracer uptake ratio (RV/LV) in $\mathrm{PAH}$ patients and patients with unoperated chronic thromboembolic PH (CTEPH) (218). BMIPP is a structurally modified fatty acid that is a probe for myocardial fatty acid utilization (306). Decreased ${ }^{123}$ I-BMIPP uptake reflects reduced fatty acid uptake and has been suggested to be due to deranged esterification to triglyceride, reduced myocardial adenosine triphosphate content (306) and impaired mitochondrial function (225). Patients also underwent gated CT scans (to assess RV ejection function and percentage systolic wall thickening) and right heart catheterization (to measure mPAP). This preliminary study demonstrated that myocardial fatty acid uptake is impaired in the failing, hypertrophied PAH RV. Later Matsushita et al. showed similar findings in patients with chronic respiratory disease and Group 3 PH (197). The Matsushita study evaluated fatty acid utilization in the RV of PAH patients using the long-chain fatty acid analogue $14(\mathrm{R}, \mathrm{S})-\left[{ }^{18} \mathrm{~F}\right]$ fluoro-6-thia-heptadecanoic acid $\left(\left[{ }^{18}\right.\right.$ F]FTHA) for PET imaging (197).

Recently, Ohiro et al. presented the first study of PET imaging to evaluate both glucose and fatty 
acid metabolism in PAH patients (226). They also correlated metabolism with both RV function and PAH severity. This study demonstrated that (i) increased RV pressure overload was associated with increased relative RV glucose uptake, (ii) the ratio of glucose to fatty acid uptake increased in direct proportion to the rise in PAP and (iii) there was a significant association between increased glucose and fatty acid uptake and declining RV function. Fatty acid utilization increased with declines in RV ejection fraction, but not PAP severity. This suggested a pathologic role for fatty acids in the development of maladaptive RVH. Figure 17 shows the myocardial perfusion imaging (MPI)-PET, FDG-PET, and FTHA-PET images in three patients categorized as having mild, moderate, or severe PAH. MPI-PET images were acquired using ${ }^{82} \mathrm{Rb}$ (Rubidium) or ${ }^{13} \mathrm{~N}$ ammonia $\left(\mathrm{NH}_{3}\right)$ as flow tracers, and FTHA-PET images were acquired with $\left[{ }^{18} \mathrm{~F}\right] \mathrm{FTHA}$ as a fatty acid metabolic tracer (197). Additionally, an exploratory analysis revealed a relative mismatch between perfusion and FDG uptake (or metabolism) in the RV in all patients with severe PAH, suggesting that the $\mathrm{RV}$ is in a state of hypernation resulting from repeatitive ischemic insults. This finding supported the hypothesis that ischemia may drive the switch to glycolysis in patients with severe PAH (226)(272).

FDG-PET imaging is also an encouraging technique to assess the efficacy of novel targeted therapeutic approaches (227). Ideally, one would want to measure FDG uptake in the diseaserelevant pulmonary vascular cells. However, regional variations in air, blood, and water fractions can lead to inaccurate estimates of FDG uptake and confound the interpretation of the PET images. To improve the accuracy in lung PET quantification and correct interpretation of the PET parameters, other independent techniques have been proposed, like successive ${ }^{15} \mathrm{O}-\mathrm{CO},{ }^{15} \mathrm{O}-\mathrm{H}_{2} \mathrm{O}$, and dynamic FDG imaging in the same scan of lungs (54). The ${ }^{15} \mathrm{O}-\mathrm{CO}$ scan is used for measuring 
the vascular volume, while ${ }^{15} \mathrm{O}-\mathrm{H}_{2} \mathrm{O}$ PET imaging is able to measure pulmonary blood flow in each lung voxel (196). Using a combination of ${ }^{15} \mathrm{O}-\mathrm{H}_{2} \mathrm{O},{ }^{15} \mathrm{O}_{2}$ and ${ }^{15} \mathrm{O}-\mathrm{CO}$ tracers, myocardial oxygen consumption can be accurately measured (317). Moreover, comparison with information from CT, lung tissue sampling, or pulmonary function testing, can provide additional context for correctly attributing PET signals to specific intrathoracic tissues. Developing appropriate algorithms for image reconstruction and motion correction are other recommendations to promote the standardization of PET acquisition and processing methodology to obtain accurate, reproducible, and interpretable images for using in clinical trials of established or novel therapies (54).

Evaluation of both lungs and heart with a single FDG-PET scan needs to choose between fasting versus glucose-loading states (277). In normal myocardium, oxidative metabolism is the primary source of energy and utilizes free fatty acids, glucose, and lactate as substrates. Myocardial PET imaging is typically performed in the glucose-loaded state, since glucose becomes the primary substrate for oxidative metabolism and insulin-driven glucose uptake increases. Thus, loading the subject with glucose prior to imaging enhances visualization of the myocardium and optimizing image quality (115). On the other hand, in the fasting state, the plasma insulin levels fall, which decreases transport of glucose into the myocytes and increases free fatty acid availability (115). The myocardium then uses fatty acids as its primary energy source. FDG-PET in the fasting state leads to lower blood glucose levels, enabling cells to capture FDG independent from hormonal influence, and therefore better reflect the true glycolytic metabolic status of cells (277). Hence, in order to investigate the glycolytic status of both lung and heart, FDG-PET imaging is best performed in the fasting state is efficient to assess RV and pulmonary glucose metabolism, 
although it does not produce the highest myocardial uptake of FDG.

In summary, novel imaging modalities with high diagnostic accuracy provide non-invasive in vivo quantitative measurements of glycolytic abnormalities in lung and heart morphology and function. Combining complementary imaging techniques, like CT, MRI, SPECT, and PET, can considerably improve our ability to image structure and function in the pulmonary vasculature

and RV in preclinical models of PAH and patients with PAH. For instance, ${ }^{18} \mathrm{~F}$-FDG PET imaging in fasting and glucose loading states can be used to evaluate RV and pulmonary glycolytic metabolism and monitor disease progression.

Therapies Targeting the Mitochondrial and Metabolic Pathways in Patients with Pulmonary Hypertension

Currently, there is no approved PH therapy that targets the mitochondrial and metabolic pathway. Preclinical animal studies have identified several promising therapeutic agents and strategies. An ideal therapy should be able to reverse established pulmonary vascular remodeling and promote adaptive right ventricular remodelling (Figure 18).

A search on ClinicalTrials.gov using the search term 'pulmonary hypertension' and filter 'metabolism OR mitochondria' returned 37 studies, but only 15 studies involved a therapeutic intervention (Figure 19). Among these 15 studies, three evaluated iron supplementation in patients with PAH (NCT03371173, NCT01447628, and NCT01847352), two tested partial inhibitors of FAO, ranolazine (NCT01839110) and trimetazidine (NCT03273387), one tested the PDK inhibitor, dichloroacetate (NCT01083524), two evaluated metformin (NCT03349775, NCT03617458), six assessed exercise and nutritional interventions (NCT03385733, 
NCT03288025, NCT02558582, NCT03476629, NCT03550729, and NCT02000856), and one study evaluated a combination of sildenafil and rehabilitation program (NCT01055405) (Figure 19).

\section{Iron Supplementation}

Iron deficiency is common in PAH and is associated with decreased exercise capacity and increased mortality $(261,267)$. However, the true iron status is difficult to determine in chronic disease states, such as PAH, due to the presence of inflammation-induced increases in ferritin and suppression of serum iron and transferrin (334). Instead, it is preferable to measure circulating soluble transferrin receptor (sTfR) levels, since it is largely unaffected by inflammation (261). Rhodes et al. used sTfR $>28.1 \mathrm{nmol} / \mathrm{L}$ as a cut-off for iron deficiency (261). Using this cut-off, the prevalence of iron deficiency in PAH patients was $63 \%$ compared to $16.7 \%$ in healthy controls (261). PAH patients with $\mathrm{sTfR}>28.1 \mathrm{nmol} / \mathrm{L}$ had significantly reduced 6-minute walk test (6MWT) $(247 \pm 154$ vs. $340 \pm 156 \mathrm{~m})$ and significantly increased mortality (261). Furthermore, HPV was enhanced in patients with iron deficiency versus controls [PASP by echocardiography increased by 16.1 vs. $10.7 \mathrm{mmHg}$, respectively; $\mathrm{p}=0.001$ ] (105). Intravenous iron supplementation caused greater reduction of the hypoxia-induced increase in PASP in the iron deficiency group compared to controls (absolute reduction 11.1 vs. $6.8 \mathrm{mmHg}$, respectively, $p=0.035$ ) (105). Hence, iron supplementation seems to be a potentially attractive therapeutic option for patients with PAH and iron deficiency.

A 12-week, cross-over study of 15 patients with $\mathrm{PAH}$, treated with a single dose of intravenous

ferric carboxymaltose (Ferinject; Vifor Pharma, Glattbrugg, Switzerland; $1000 \mathrm{mg}$, in $20 \mathrm{~mL}$ of 
$\mathrm{NaCl} 0.9 \%$ over 2 hours) found iron therapy improved exercise capacity (time to anaerobic threshold $175 \pm 33$ s before vs. $238 \pm 43 \mathrm{~s}$ after iron treatment, $p<0.001$ ) and increased quality of life questionnaire $(49 \pm 10 \%$ before vs. $60 \pm 17 \%$ after; $p<0.001)$ versus placebo $(268)$. There was a statistically insignificant trend toward improvement of 6 MWT $(409 \pm 110 \mathrm{~m}$ before vs. $428 \pm 94 \mathrm{~m}$ after, $p=0.07$ ) (268) but no change in RV function, as measured by cardiac MRI before and after iron treatment. However, iron therapy improved skeletal muscle oxygen transport (myoglobin concentration $0.34 \pm 0.17 \mathrm{mM}$ before vs. $0.44 \pm 0.11 \mathrm{mM}$ after, $p<0.05)(268)$.

Two clinical trials are currently underway to test the effectiveness of iron supplementation in patients with PH. ORION-PH-1 is a Phase 3 study testing oral iron supplementation (ferric maltol, $30 \mathrm{mg}$ twice daily for 12 weeks). Another Phase 2 study tests a single dose of intravenous iron (Ferinject; Vifor Pharma, Glattbrugg, Switzerland or CosmoFer; Pharmacosmos, Reading, Berkshire, UK $1000 \mathrm{mg}$ ) with the endpoints being pulmonary vascular resistance and exercise capacity, measured 12-weeks post a single iron treatment.

\section{Fatty acid oxidation inhibitors}

Ranolazine (49) and trimetazidine (66) are approved therapies for chronic angina pectoris (in the USA) and heart failure (in Europe), respectively. Both drugs partially inhibit fatty acid oxidation (FAO), and thereby activate the Randle cycle to promote glucose oxidation, which uses oxygen more efficiently than FAO $(96,339)$. This metabolic shift is favourable in the setting of chronic myocardial ischemia, as impaired blood flow leads to reduced oxygen supply to the myocardium (339). 
In PAH, increased pulmonary vascular resistance leads to increased RV pressure. However, the aortic pressure is usually unchanged or reduced, whilst RV pressure is very high. This mismatch reduces the epicardial perfusion pressure (aortic pressure $-\mathrm{RV}$ pressure) in the right coronary artery which serves the RV, contributing to RV ischemia (Figure 20). Hence, therapies, such as ranolazine and trimetazidine may be repurposed to treat RV dysfunction in PH. Indeed, both preclinical animal data and small clinical studies testing ranolazine and trimetazidine are promising $(96,110,112,154,264)$.

Ranolazine selectively inhibits the late sodium current ( $\left.\mathrm{I}_{\mathrm{NaL}}\right)$ at low dose and FAO at high dose. It has been hypothesized that altering $\mathrm{I}_{\mathrm{NaL}}$ and/or inhibiting FAO can improve RV function and exercise capacity (112). We confirmed the ability of this agent to inhibit FAO, but saw no effect on QRS or QTc duration, arguing against a significant role for sodium channel blockers (96). In MCT-PAH, ranolazine treatment, started one week after MCT injection, caused dose-dependent reductions in RV pressure, RV hypertrophy, and B-type natriuretic peptide level (178). Rocchetti et al. initiated ranolazine (30 mg/kg IP BID for three weeks) beginning two days after MCT injection. Ranolazine reduced MCT-induced PA medial thickening by $20 \%$, decreased RVSP by $45 \%$, and reduced RV wall thickness (MCT $0.76 \pm 0.03, \mathrm{n}=13$ vs. Ranolazine $+\mathrm{MCT} 0.56 \pm 0.03$, $\mathrm{n}=10, \mathrm{p}<0.05$ ) (264). Furthermore, ranolazine prevented both the $\mathrm{I}_{\mathrm{NaL}}$ enhancement and corresponding increase in $\mathrm{Ca}^{2+}$ release from RV myocytes T-tubule seen in the MCT group (264). Since ranolazine reduces PA medial thickening, as well as improving RV function, these studies cannot definitively discriminate the direct effects of ranolazine on RV function versus mixed effects of the therapy on the pulmonary vasculature and RV. 
To see whether ranolazine has direct effect on the RV, Fang et al. used the pulmonary artery banding (PAB) rat model of RV pressure-volume overload, which has normal pulmonary vasculature (96). PAB rats treated with ranolazine $(20 \mathrm{mg} /$ day administered orally for one week, beginning three weeks post-PAB) have increased cardiac output and exercise capacity and attenuated exertional lactic acidemia (96). Fang et al. showed that ranolazine improves RV function by partially inhibiting FAO, which indirectly increases glucose oxidation via the Randle cycle (Figure 21) (96). In addition, Fang et al. tested trimetazidine $(0.7 \mathrm{~g} / \mathrm{L}$ drinking water for one week, beginning three weeks post-PAB), which inhibits the mitochondrial enzyme long-chain 3keoacyl CoA thiolase (3-KAT) (96). Similar to ranolazine, trimetazidine reduces RV hypertrophy and improves RV function. Moreover, trimetazidine shortens the prolonged QTc interval seen in PAB rats (96).

The evidence from these animal studies has led to several small human studies. Gomberg-Maitland et al. performed a Phase 1 safety study of ranolazine in 12 PAH patients (six ranolazine and six placebo) treated with ranolazine (500 mg PO BID for the first four weeks and then up-titrated to $1000 \mathrm{mg}$ PO BID for another eight weeks) (110). Among the six patients receiving ranolazine, two patients had early withdrawal due to adverse events (mainly gastrointestinal complaints) and only one patient had therapeutic ranolazine serum levels (110). All patients in the placebo group completed the 12-week study (110). In a cross-over study, Khan et al. tested ranolazine (1000 mg PO BID) in 11 symptomatic PAH patients for three months (154). Among the 11 patients, one patient discontinued ranolazine after three days, due to drug-drug interaction, eight patients were able to tolerate ranolazine $1000 \mathrm{mg}$ PO BID for three months, and two patients required a dose reduction to $500 \mathrm{mg}$ PO BID due to adverse effects (154). After three months of ranolazine treatment, test subjects reported improved WHO functional class $(\mathrm{p}=0.0013)$ and objective testing 
revealed reduced $\mathrm{RV}$ end-diastolic $(\mathrm{p}=0.01)$ and end-systolic $(\mathrm{p}=0.0002)$ areas, and improved tricuspid annular plane systolic excursion (TAPSE) $(\mathrm{p}=0.015)$ on echocardiographic measurements compared to their initial baseline (154). However, there was no improvement in invasive hemodynamic parameters compared to baseline (154). These two small studies are not conclusive. Currently, there are two randomized and placebo-controlled clinical studies testing ranolazine (NCT01839110) and trimetazidine (NCT03273387) underway in patients with PAH.

\section{Dichloroacetate}

Dichloroacetate (DCA) is a metabolic modulator that inhibits all four PDK isoforms (295). Inhibiting PDK enhances PDH activity, which increases the pyruvate/lactate ratio and reverses the reduced redox state seen in the PASMC of rats with chronic HPH (208) (Figure 22). Furthermore, by promoting an oxidized state, DCA increases whole cell $I_{\mathrm{K}}$ current in PASMC and enhances the expression of Kv2.1 channel and reverses HPH (208).

McMurtry et al. tested DCA in MCT-PAH. DCA improves the survival of MCT rats, normalizes the pulmonary vascular resistance, and reverses pulmonary arterial remodeling (202). Expression of both Kv1.5 and Kv2.1 channels is downregulated in the PASMC from MCT rats, leading to membrane depolarization, calcium overload and apoptosis resistance. DCA treatment reverses the Kv1.5 downregulation (202). In addition, DCA increases the apoptosis/proliferation ratio in the pulmonary arterial media and attenuates pulmonary arterial medial thickening (202). DCAinduced Kv1.5 upregulation and increased PASMC apoptosis are mediated via mitochondrialderived increase of $\mathrm{H}_{2} \mathrm{O}_{2}$ production (202). 
Based on the above animal studies, Michelakis et al. tested DCA in a Phase 1 clinical trial (206). Twenty patients with IPAH were treated with DCA in this dose-finding trial of 3, 6.25, or 12.5 $\mathrm{mg} / \mathrm{kg}$ orally twice daily for four months (NCT01083524) (206). These patients were all on sildenafil, 13 on endothelin receptor antagonist (ERA) in addition to sildenafil, and one patient on parenteral prostanoid plus ERA and sildenafil (206). By four months, four patients had withdrawn from the study, and the other 16 patients completing the trial were taking 3 or $6.25 \mathrm{mg} / \mathrm{kg}$ PO BID (206). As a result, $6.25 \mathrm{mg} / \mathrm{kg}$ PO BID was established as the highest tolerable dose (206). The most common adverse reaction was peripheral paresthesia, which was reversible and dosedependent (206). During the four-month period, no patients required hospitalization, deteriorated clinically, or had decreased 6-minute walk distance by $10 \%$ (206). These data suggest DCA is safe to use as an add-on therapy to currently approved PAH therapies, although a larger study is required.

In terms of effectiveness, four months of DCA treatment significantly reduced mPAP (pre-DCA $49 \pm 3$ vs. post-DCA $45 \pm 3 \mathrm{mmHg}, p<0.05$ ), pulmonary vascular resistance (pre-DCA $719 \pm 107$ vs. post-DCA $649 \pm 102$ dynes ${ }^{*} \mathrm{~s} / \mathrm{cm}^{5}, p<0.05$ ), and improved 6 MWT (pre-DCA $400 \pm 28$ vs. post-DCA $425 \pm 23 \mathrm{~m}, p<0.05$ ) (206). However, the response to DCA varied considerably amongst the patients. The differential response to DCA was attributed to polymorphisms in the SIRT3, a gene encoding a mitochondrial deacetylase, which can activate PDH (242), and UCP2, a gene encoding a mitochondrial protein that regulates calcium entry $(84,206)$. The single nucleotide polymorphism (SNP) for SIRT3 rs11246020 A allele causes a change of valine to isoleucine at residue 208, which causes a $34 \%$ reduction in SIRT3 activity compared to the G allele (137). Similarly, the UCP2 rs659366 G allele is associated with reduced transcription compared to the A allele $(92,320)$. Disrupting SIRT3 and/or UCP2 function inhibits PDH activity by PDK- 
independent mechanisms (which are thus not amenable to DCA therapy). Michelakis et al. developed a SNP score where one point is given for each loss-of-function variant for SIRT3 and UCP2: Zero means that both the variants are absent, a score of four means that both variants are present, and a score in between means variants are presented in various heterozygous manners (206). As expected, patients with high SNP score had poor response to DCA, whereas patients with SNP score of 0 or 1 had better responses to DCA (206). This study highlights the importance of considering patient's genotype when designing clinical trial and attests to the potential benefits of personalized medicine.

\section{Metformin}

Approximately $50 \%$ of patients with heart failure with preserved ejection fraction (HFpEF) develop PH (119). Currently, there is no evidence-based therapy for HFpEF or PH associated with HFpEF (265). A major obstacle to therapeutic innovation for HFpEF is the lack of preclinical models that accurately recapitulate the pathophysiological and clinical manifestations of HFpEF (265). Schiattarella et al. developed a mouse model of HFpEF and discovered a pathologic role for the inositol-requiring protein $1 \alpha(\operatorname{IRE} 1 \alpha)$-X-box-binding protein 1 (XBP1s) pathway, which is an evolutionarily conserved pathway involved in intracellular protein folding and enhancing secretory capacity (278). To develop HFpEF, C57BL/6N wild-type mice were treated with high fat diet (metabolic stress) and No-nitro-L-arginine methyl ester (L-NAME), which inhibits nitric oxide production and causes systemic hypertension (mechanical stress) (278). This multi-hit model mimics the clinical scenario in HFpEF patients, who often have multiple comorbidities including obesity, hypertension, and diabetes (Figure 23) (282). Consistent with this observation, Ranchoux et al. recently developed a "multi-hit" preclinical model of group $2 \mathrm{PH}$ which combines mild 
diastolic dysfunction induced by supra-aortic banding (SAB) and metabolic syndrome (MetS) induced by high-fat diet and olanzapine treatment (anti-psychotic drug known to induce MetS). In their model, the combination of MetS and diastolic dysfunction is required to induce significant PH and adverse pulmonary vascular remodeling (256). At the molecular level, they showed that increased inflammation (characterized by macrophage accumulation and elevated IL-6 levels) as well leptin accumulation contributes to the etiology of group $2 \mathrm{PH}$ in both the preclinical model and human patients. Then, they showed that Metformin, a first line diabetic medication, which is also known to inhibit leptin secretion and have an anti-inflammatory effects, reverse adverse pulmonary vascular lesion and improve group $2 \mathrm{PH}(41,212)$.

Schiattarella et al. provided mechanistic insights for the pathophysiology of HFpEF. Most notably, the 'two-hit' theory of both metabolic and mechanical stress are required to cause HFpEF (278). Recently, a large randomized, double-blinded, placebo-controlled trial tested sodium-glucose cotransporter 2 (SGLT2) inhibitor, canagliflozin, was conducted in patients with diabetes and nephropathy. The results showed that canagliflozin markedly reduces the risk of hospitalization for heart failure (hazard ratio, $0.61 ; 95 \% \mathrm{CI}, 0.47$ to $0.80 ; p<0.001$ ) (243). Although this study did not differentiate $\mathrm{HFpEF}$ and heart failure with reduced ejection fraction ( $\mathrm{HFrEF})$, it provides partial evidence that supports the 'two-hit' theory, in which targeting the metabolic stress is an effective strategy in treating HFpEF. Hence, treating patients with metformin, a first line diabetes medication which also targets metabolic stress, may be effective against $\mathrm{HFpEF}$ and/or $\mathrm{PH}$ associated with HFpEF. A Phase II clinical trial testing metformin for PH and HFpEF is currently underway (NCT03629340).

\section{Exercise and Nutrition}


There was traditionally concern about promoting exercise training for patients with $\mathrm{PAH}$, given their poor RV functional reserve and predisposition to exertional syncope. This concern was dispelled by Mereles et al., who showed that respiratory and physical training improves exercise capacity (increased 6MWT by $111 \mathrm{~m} 95 \%$ CI, 65 to $139 \mathrm{~m}$; $\mathrm{p}<0.001$ ) and improved quality of life in patients with severe PAH (204). Subsequent studies on patients with different types of secondary $\mathrm{PH}$ also demonstrated that exercise training can improve exercise capacity, muscle function, peak oxygen consumption and quality of life $(32,89,116-118,152,219)$. Thus, substantial evidence suggests that exercise training is beneficial even in patients with severe $\mathrm{PH}$. New clinical trials are trying to find the optimal combination of different types of physical activity and respiratory muscle training.

Can nutritional interventions benefit PAH patients by altering mitochondrial metabolism? The Pulmonary Hypertension Association published a book entitled: Pulmonary Hypertension: A Patient's Survival Guide (130). In this book, there is a section on diet and nutrition mainly based on expert recommendations, such as controlling salt intake, eating an iron rich diet, and limiting alcohol and caffeine intake (130). A randomized, double-blinded, placebo-controlled, cross-over study by Henrohn et al. showed that oral supplementation with nitrate-rich beetroot juice ( 16 mmol nitrate per day) for one week increases pulmonary NO production (measured using exhaled NO, plasma and salivary nitrate and nitrite) compared to placebo (133). Furthermore, there was a trend towards improved right ventricular function and peak power output to peak oxygen consumption in patients who had beetroot juice (133). More well-designed studies similar to this one is required to find out which diet can actually benefit patients with $\mathrm{PH}$. 
Among the few clinical trials testing interventions targeting the metabolic and mitochondrial pathways to treat pulmonary hypertension, a common focus is on restoring normal metabolism. These strategies work well in preclinical animal models. However, pulmonary hypertension is a complex and heterogeneous disease in human. Hence, one therapy that works for some, may not work for others. We will wait and see how well these promising treatment strategies translate into clinical therapies for patients with pulmonary hypertension in ongoing clinical trials. Moving forward, with better understanding of the underlying pathomolecular mechanisms of pulmonary hypertension, future pulmonary hypertension clinical trials should have a focus on personalized medicine, so the right therapeutic strategies can be applied to the right patients.

\section{Conclusion}

The role of mitochondria in the complexity of lung biology is receiving increasing attention and acquired mitochondrial dysfunction is now widely accepted as a contributing mechanism to many aspects of pulmonary vascular disease in PAH (Figure 24). Despite extensive research, there is no cure for diseases involving the pulmonary vasculature such as pulmonary hypertension. Although growing evidence indicates reversible mitochondrial abnormalities in $\mathrm{PH}$, current therapies are not designed to target dysregulated mitochondria or metabolic abnormalities caused by these dysfunctions. Dysregulated mitochondrial dynamics and impaired mitochondrial metabolism in PAH have been experimentally targeted in numerous preclinical studies and some early clinical human trials, with promising results. Mitochondrial modulators appear to have the potential to address a largely untargeted aspect of the PAH phenotype, namely the hyperproliferative, apoptosis-resistant phenotype while also improving RV function. Further understanding of the role of mitochondria in the physiology of pulmonary vasculature and identification of potential 
molecular and pharmacological tools to target mitochondrial dysfunction, and measure mitochondrial function in vivo, using mitochondrial-targeted molecular imaging probes, holds promise for PAH patients. However, careful and controlled preclinical and clinical studies will be required to evaluate the safety and therapeutic value of these new therapies. 


\section{References}

1. Adaniya SM, J OU, Cypress MW, Kusakari Y, and Jhun BS. Posttranslational modifications of mitochondrial fission and fusion proteins in cardiac physiology and pathophysiology. Am J Physiol Cell Physiol 316: C583-C604, 2019.

2. Aggarwal S, Mannam P, and Zhang J. Differential regulation of autophagy and mitophagy in pulmonary diseases. American journal of physiology Lung cellular and molecular physiology 311: L433-452, 2016.

3. Ahmadi A, Ohira H, and Mielniczuk LM. FDG PET imaging for identifying pulmonary hypertension and right heart failure. Curr Cardiol Rep 17: 555, 2015.

4. Aljubran SA, Cox R, Jr., Tamarapu Parthasarathy P, Kollongod Ramanathan G, Rajanbabu V, Bao H, Mohapatra SS, Lockey R, and Kolliputi N. Enhancer of zeste homolog 2 induces pulmonary artery smooth muscle cell proliferation. PloS one 7: e37712, 2012.

5. Ameli-Renani S, Rahman F, Nair A, Ramsay L, Bacon JL, Weller A, Sokhi HK, Devaraj A, Madden B, and Vlahos I. Dual-energy CT for imaging of pulmonary hypertension: challenges and opportunities. Radiographics 34: 1769-1790, 2014.

6. Ameshima S, Golpon H, Cool CD, Chan D, Vandivier RW, Gardai SJ, Wick M, Nemenoff RA, Geraci MW, and Voelkel NF. Peroxisome proliferator-activated receptor gamma (PPARgamma) expression is decreased in pulmonary hypertension and affects endothelial cell growth. Circ Res 92: 1162-1169, 2003.

7. Antico Arciuch VG, Elguero ME, Poderoso JJ, and Carreras MC. Mitochondrial regulation of cell cycle and proliferation. Antioxid Redox Signal 16: 1150-1180, 2012.

8. Archer SL. Mitochondrial dynamics--mitochondrial fission and fusion in human diseases. N Engl J Med 369: 2236-2251, 2013.

9. Archer SL. Mitochondrial fission and fusion in human diseases. $N$ Engl J Med 370: 1074, 2014.

10. Archer SL. Pyruvate Kinase and Warburg Metabolism in Pulmonary Arterial Hypertension: Uncoupled Glycolysis and the Cancer-Like Phenotype of Pulmonary Arterial Hypertension. Circulation 136: 2486-2490, 2017.

11. Archer SL, Fang YH, Ryan JJ, and Piao L. Metabolism and bioenergetics in the right ventricle and pulmonary vasculature in pulmonary hypertension. Pulm Circ 3: 144-152, 2013.

12. Archer SL, Gomberg-Maitland M, Maitland ML, Rich S, Garcia JG, and Weir EK. Mitochondrial metabolism, redox signaling, and fusion: a mitochondria-ROS-HIF-1alphaKv1.5 02-sensing pathway at the intersection of pulmonary hypertension and cancer. Am J Physiol Heart Circ Physiol 294: H570-578, 2008.

13. Archer SL, Huang J, Henry T, Peterson D, and Weir EK. A redox-based 02 sensor in rat pulmonary vasculature. Circ Res 73: 1100-1112, 1993.

14. Archer SL, London B, Hampl V, Wu X, Nsair A, Puttagunta L, Hashimoto K, Waite RE, and Michelakis ED. Impairment of hypoxic pulmonary vasoconstriction in mice lacking the voltage-gated potassium channel Kv1.5. FASEB journal : official publication of the Federation of American Societies for Experimental Biology 15: 1801-1803, 2001. 15. Archer SL, Marsboom G, Kim GH, Zhang HJ, Toth PT, Svensson EC, Dyck JR, Gomberg-Maitland M, Thebaud B, Husain AN, Cipriani N, and Rehman J. Epigenetic attenuation of mitochondrial superoxide dismutase 2 in pulmonary arterial hypertension: a 
basis for excessive cell proliferation and a new therapeutic target. Circulation 121: 26612671, 2010.

16. Archer SL, Nelson DP, and Weir EK. Detection of activated 02 species in vitro and in rat lungs by chemiluminescence. J Appl Physiol (1985) 67: 1912-1921, 1989.

17. Archer SL, Nelson DP, and Weir EK. Simultaneous measurement of 02 radicals and pulmonary vascular reactivity in rat lung. J Appl Physiol (1985) 67: 1903-1911, 1989. 18. Archer SL, Souil E, Dinh-Xuan AT, Schremmer B, Mercier JC, El Yaagoubi A, Nguyen-Huu L, Reeve HL, and Hampl V. Molecular identification of the role of voltagegated K+ channels, Kv1.5 and Kv2.1, in hypoxic pulmonary vasoconstriction and control of resting membrane potential in rat pulmonary artery myocytes. The Journal of clinical investigation 101: 2319-2330, 1998.

19. Archer SL, Will JA, and Weir EK. Redox status in the control of pulmonary vascular tone. Herz 11: 127-141, 1986.

20. Archer SL, Wu XC, Thebaud B, Moudgil R, Hashimoto K, and Michelakis ED. 02 sensing in the human ductus arteriosus: redox-sensitive $\mathrm{K}+$ channels are regulated by mitochondria-derived hydrogen peroxide. Biological chemistry 385: 205-216, 2004.

21. Archer SL, Wu XC, Thebaud B, Nsair A, Bonnet S, Tyrrell B, McMurtry MS, Hashimoto K, Harry G, and Michelakis ED. Preferential expression and function of voltage-gated, 02 -sensitive $\mathrm{K}+$ channels in resistance pulmonary arteries explains regional heterogeneity in hypoxic pulmonary vasoconstriction: ionic diversity in smooth muscle cells. Circ Res 95: 308-318, 2004.

22. Armulik A, Genove G, and Betsholtz C. Pericytes: developmental, physiological, and pathological perspectives, problems, and promises. Dev Cell 21: 193-215, 2011.

23. Arnoult D, Grodet A, Lee YJ, Estaquier J, and Blackstone C. Release of OPA1 during apoptosis participates in the rapid and complete release of cytochrome $c$ and subsequent mitochondrial fragmentation.J Biol Chem 280: 35742-35750, 2005.

24. Ascha M, Renapurkar RD, and Tonelli AR. A review of imaging modalities in pulmonary hypertension. Ann Thorac Med 12: 61-73, 2017.

25. Ashrafi G, and Schwarz TL. The pathways of mitophagy for quality control and clearance of mitochondria. Cell Death Differ 20: 31-42, 2013.

26. Assad TR, Hemnes AR, Larkin EK, Glazer AM, Xu M, Wells QS, Farber-Eger EH, Sheng Q, Shyr Y, Harrell FE, Newman JH, and Brittain EL. Clinical and Biological Insights Into Combined Post- and Pre-Capillary Pulmonary Hypertension. J Am Coll Cardiol 68: 2525-2536, 2016.

27. Atkins K, Dasgupta A, Chen KH, Mewburn J, and Archer SL. The role of Drp1 adaptor proteins MiD49 and MiD51 in mitochondrial fission: implications for human disease. Clin Sci (Lond) 130: 1861-1874, 2016.

28. Banes-Berceli AK, Ketsawatsomkron P, Ogbi S, Patel B, Pollock DM, and Marrero MB. Angiotensin II and endothelin-1 augment the vascular complications of diabetes via JAK2 activation. Am J Physiol Heart Circ Physiol 293: H1291-1299, 2007.

29. Baraka AS, Taha SK, and Yaacoub CI. Alarming hypoxemia during one-lung ventilation in a patient with respiratory bronchiolitis-associated interstitial lung disease. Canadian journal of anaesthesia = Journal canadien d'anesthesie 50: 411-414, 2003.

30. Bartsch P, and Swenson ER. Clinical practice: Acute high-altitude illnesses. $N$ Engl J Med 368: 2294-2302, 2013.

31. Baughman JM, Perocchi F, Girgis HS, Plovanich M, Belcher-Timme CA, Sancak 
Y, Bao XR, Strittmatter L, Goldberger 0, Bogorad RL, Koteliansky V, and Mootha VK. Integrative genomics identifies MCU as an essential component of the mitochondrial calcium uniporter. Nature 476: 341-345, 2011.

32. Becker-Grunig T, Klose H, Ehlken N, Lichtblau M, Nagel C, Fischer C, Gorenflo M, Tiede H, Schranz D, Hager A, Kaemmerer H, Miera O, Ulrich S, Speich R, Uiker S, and Grunig E. Efficacy of exercise training in pulmonary arterial hypertension associated with congenital heart disease. International journal of cardiology 168: 375-381, 2013.

33. Bertero T, Oldham WM, Cottrill KA, Pisano S, Vanderpool RR, Yu Q, Zhao J, Tai Y, Tang Y, Zhang YY, Rehman S, Sugahara M, Qi Z, Gorcsan J, 3rd, Vargas SO, Saggar R, Saggar R, Wallace WD, Ross DJ, Haley KJ, Waxman AB, Parikh VN, De Marco T, Hsue PY, Morris A, Simon MA, Norris KA, Gaggioli C, Loscalzo J, Fessel J, and Chan SY. Vascular stiffness mechanoactivates YAP/TAZ-dependent glutaminolysis to drive pulmonary hypertension. J Clin Invest 126: 3313-3335, 2016.

34. Bokhari S, Raina A, Rosenweig EB, Schulze PC, Bokhari J, Einstein AJ, Barst RJ, and Johnson LL. PET imaging may provide a novel biomarker and understanding of right ventricular dysfunction in patients with idiopathic pulmonary arterial hypertension. Circ Cardiovasc Imaging 4: 641-647, 2011.

35. Bonnet S, Archer SL, Allalunis-Turner J, Haromy A, Beaulieu C, Thompson R, Lee CT, Lopaschuk GD, Puttagunta L, Bonnet S, Harry G, Hashimoto K, Porter CJ, Andrade MA, Thebaud B, and Michelakis ED. A mitochondria-K+ channel axis is suppressed in cancer and its normalization promotes apoptosis and inhibits cancer growth. Cancer Cell 11: 37-51, 2007.

36. Bonnet S, Michelakis ED, Porter CJ, Andrade-Navarro MA, Thebaud B, Bonnet S, Haromy A, Harry G, Moudgil R, McMurtry MS, Weir EK, and Archer SL. An abnormal mitochondrial-hypoxia inducible factor-1alpha-Kv channel pathway disrupts oxygen sensing and triggers pulmonary arterial hypertension in fawn hooded rats: similarities to human pulmonary arterial hypertension. Circulation 113: 2630-2641, 2006.

37. Boucherat O, Chabot S, Paulin R, Trinh I, Bourgeois A, Potus F, Lampron MC, Lambert C, Breuils-Bonnet S, Nadeau V, Paradis R, Goncharova EA, Provencher S, and Bonnet S. HDAC6: A Novel Histone Deacetylase Implicated in Pulmonary Arterial Hypertension. Sci Rep 7: 4546, 2017.

38. Bowers R, Cool C, Murphy RC, Tuder RM, Hopken MW, Flores SC, and Voelkel

NF. Oxidative stress in severe pulmonary hypertension. American journal of respiratory and critical care medicine 169: 764-769, 2004.

39. Bradford JR, and Dean HP. The Pulmonary Circulation. The Journal of physiology 16: 34-158 125, 1894.

40. Bromberg JF, Horvath CM, Besser D, Lathem WW, and Darnell JE, Jr. Stat3 activation is required for cellular transformation by v-src. Mol Cell Biol 18: 2553-2558, 1998.

41. Cameron AR, Morrison VL, Levin D, Mohan M, Forteath C, Beall C, McNeilly AD, Balfour DJ, Savinko T, Wong AK, Viollet B, Sakamoto K, Fagerholm SC, Foretz M, Lang CC, and Rena G. Anti-Inflammatory Effects of Metformin Irrespective of Diabetes Status. Circ Res 119: 652-665, 2016.

42. Can MM, Kaymaz C, Tanboga IH, Tokgoz HC, Canpolat N, Turkyilmaz E, Sonmez $\mathbf{K}$, and Ozdemir $\mathbf{N}$. Increased right ventricular glucose metabolism in patients with pulmonary arterial hypertension. Clin Nucl Med 36: 743-748, 2011. 
43. Cao Y, Zhang X, Wang L, Yang Q, Ma Q, Xu J, Wang J, Kovacs L, Ayon RJ, Liu Z, Zhang M, Zhou Y, Zeng X, Xu Y, Wang Y, Fulton DJ, Weintraub NL, Lucas R, Dong Z, Yuan JX, Sullivan JC, Meadows L, Barman SA, Wu C, Quan J, Hong M, Su Y, and Huo Y. PFKFB3-mediated endothelial glycolysis promotes pulmonary hypertension. Proc Natl Acad Sci U S A 2019.

44. Cao Y, Zhang X, Wang L, Yang Q, Ma Q, Xu J, Wang J, Kovacs L, Ayon RJ, Liu Z, Zhang M, Zhou Y, Zeng X, Xu Y, Wang Y, Fulton DJ, Weintraub NL, Lucas R, Dong Z, Yuan JX, Sullivan JC, Meadows L, Barman SA, Wu C, Quan J, Hong M, Su Y, and Huo Y. PFKFB3-mediated endothelial glycolysis promotes pulmonary hypertension. Proc Natl Acad Sci U S A 116: 13394-13403, 2019.

45. Caruso P, Dunmore BJ, Schlosser K, Schoors S, Dos Santos C, Perez-Iratxeta C, Lavoie JR, Zhang H, Long L, Flockton AR, Frid MG, Upton PD, D'Alessandro A, Hadinnapola C, Kiskin FN, Taha M, Hurst LA, Ormiston ML, Hata A, Stenmark KR, Carmeliet P, Stewart DJ, and Morrell NW. Identification of MicroRNA-124 as a Major Regulator of Enhanced Endothelial Cell Glycolysis in Pulmonary Arterial Hypertension via PTBP1 (Polypyrimidine Tract Binding Protein) and Pyruvate Kinase M2. Circulation 136: 2451-2467, 2017.

46. Caruso P, MacLean MR, Khanin R, McClure J, Soon E, Southgate M, MacDonald RA, Greig JA, Robertson KE, Masson R, Denby L, Dempsie Y, Long L, Morrell NW, and Baker AH. Dynamic changes in lung microRNA profiles during the development of pulmonary hypertension due to chronic hypoxia and monocrotaline. Arterioscler Thromb Vasc Biol 30: 716-723, 2010.

47. Cassidy-Stone A, Chipuk JE, Ingerman E, Song C, Yoo C, Kuwana T, Kurth MJ, Shaw JT, Hinshaw JE, Green DR, and Nunnari J. Chemical inhibition of the mitochondrial division dynamin reveals its role in Bax/Bak-dependent mitochondrial outer membrane permeabilization. Dev Cell 14: 193-204, 2008.

48. Cereghetti GM, Stangherlin A, Martins de Brito O, Chang CR, Blackstone C, Bernardi P, and Scorrano L. Dephosphorylation by calcineurin regulates translocation of Drp1 to mitochondria. Proc Natl Acad Sci U S A 105: 15803-15808, 2008.

49. Chaitman BR, Skettino SL, Parker JO, Hanley P, Meluzin J, Kuch J, Pepine CJ, Wang W, Nelson JJ, Hebert DA, Wolff AA, and Investigators M. Anti-ischemic effects and long-term survival during ranolazine monotherapy in patients with chronic severe angina.J Am Coll Cardiol 43: 1375-1382, 2004.

50. Chan SY, Zhang YY, Hemann C, Mahoney CE, Zweier JL, and Loscalzo J.

MicroRNA-210 controls mitochondrial metabolism during hypoxia by repressing the ironsulfur cluster assembly proteins ISCU1/2. Cell Metab 10: 273-284, 2009.

51. Chandel NS, McClintock DS, Feliciano CE, Wood TM, Melendez JA, Rodriguez AM, and Schumacker PT. Reactive oxygen species generated at mitochondrial complex III stabilize hypoxia-inducible factor-1alpha during hypoxia: a mechanism of 02 sensing. J Biol Chem 275: 25130-25138, 2000.

52. Chang CR, and Blackstone C. Cyclic AMP-dependent protein kinase phosphorylation of Drp1 regulates its GTPase activity and mitochondrial morphology. J Biol Chem 282: 21583-21587, 2007.

53. Chapman-Smith A, Lutwyche JK, and Whitelaw ML. Contribution of the Per/Arnt/Sim (PAS) domains to DNA binding by the basic helix-loop-helix PAS transcriptional regulators. J Biol Chem 279: 5353-5362, 2004. 
54. Chen DL, Cheriyan J, Chilvers ER, Choudhury G, Coello C, Connell M, Fisk M, Groves AM, Gunn RN, Holman BF, Hutton BF, Lee S, MacNee W, Mohan D, Parr D, Subramanian D, Tal-Singer R, Thielemans K, van Beek EJ, Vass L, Wellen JW, Wilkinson I, and Wilson FJ. Quantification of Lung PET Images: Challenges and Opportunities. J Nucl Med 58: 201-207, 2017.

55. Chen H, Chomyn A, and Chan DC. Disruption of fusion results in mitochondrial heterogeneity and dysfunction. J Biol Chem 280: 26185-26192, 2005.

56. Chen H, Detmer SA, Ewald AJ, Griffin EE, Fraser SE, and Chan DC. Mitofusins Mfn1 and Mfn2 coordinately regulate mitochondrial fusion and are essential for embryonic development. J Cell Biol 160: 189-200, 2003.

57. Chen H, Vermulst M, Wang YE, Chomyn A, Prolla TA, McCaffery JM, and Chan

DC. Mitochondrial fusion is required for mtDNA stability in skeletal muscle and tolerance of mtDNA mutations. Cell 141: 280-289, 2010.

58. Chen KH, Dasgupta A, Ding J, Indig FE, Ghosh $\mathbf{P}$, and Longo DL. Role of mitofusin 2 (Mfn2) in controlling cellular proliferation. FASEB journal : official publication of the Federation of American Societies for Experimental Biology 28: 382-394, 2014.

59. Chen KH, Dasgupta A, Lin J, Potus F, Bonnet S, Iremonger J, Fu J, Mewburn J, Wu D, Dunham-Snary K, Theilmann AL, Jing ZC, Hindmarch C, Ormiston ML, Lawrie A, and Archer SL. Epigenetic Dysregulation of the Dynamin-Related Protein 1 Binding Partners MiD49 and MiD51 Increases Mitotic Mitochondrial Fission and Promotes Pulmonary Arterial Hypertension: Mechanistic and Therapeutic Implications. Circulation 138: 287-304, 2018.

60. Chen KH, Guo X, Ma D, Guo Y, Li Q, Yang D, Li P, Qiu X, Wen S, Xiao RP, and Tang J. Dysregulation of HSG triggers vascular proliferative disorders. Nat Cell Biol 6: 872-883, 2004.

61. Chen Y, and Dorn GW, 2nd. PINK1-phosphorylated mitofusin 2 is a Parkin receptor for culling damaged mitochondria. Science 340: 471-475, 2013.

62. Cherok E, Xu S, Li S, Das S, Meltzer WA, Zalzman M, Wang C, and Karbowski M. Novel regulatory roles of Mff and Drp1 in E3 ubiquitin ligase MARCH5-dependent degradation of MiD49 and Mcl1 and control of mitochondrial dynamics. Mol Biol Cell 28: 396-410, 2017.

63. Chettimada S, Gupte R, Rawat D, Gebb SA, McMurtry IF, and Gupte SA. Hypoxiainduced glucose-6-phosphate dehydrogenase overexpression and -activation in pulmonary artery smooth muscle cells: implication in pulmonary hypertension. American journal of physiology Lung cellular and molecular physiology 308: L287-300, 2015.

64. Chinnery PF, and Hudson G. Mitochondrial genetics. Br Med Bull 106: 135-159, 2013.

65. Church SL, Grant JW, Meese EU, and Trent JM. Sublocalization of the gene encoding manganese superoxide dismutase (MnSOD/SOD2) to 6q25 by fluorescence in situ hybridization and somatic cell hybrid mapping. Genomics 14: 823-825, 1992.

66. Ciapponi A, Pizarro R, and Harrison J. Trimetazidine for stable angina. Cochrane Database of Systematic Reviews 2005.

67. Cicone F, Viertl D, Quintela Pousa AM, Denoel T, Gnesin S, Scopinaro F, Vozenin MC, and Prior JO. Cardiac Radionuclide Imaging in Rodents: A Review of Methods, Results, and Factors at Play. Front Med (Lausanne) 4: 35, 2017.

68. Cipolat S, Martins de Brito 0, Dal Zilio B, and Scorrano L. OPA1 requires 
mitofusin 1 to promote mitochondrial fusion. Proc Natl Acad Sci U S A 101: 15927-15932, 2004.

69. Coalson JJ. Pathology of bronchopulmonary dysplasia. Semin Perinatol 30: 179-184, 2006.

70. Cohen MM, Leboucher GP, Livnat-Levanon N, Glickman MH, and Weissman AM. Ubiquitin-proteasome-dependent degradation of a mitofusin, a critical regulator of mitochondrial fusion. Mol Biol Cell 19: 2457-2464, 2008.

71. Cornfield DN, Martin EB, Hampl V, and Archer SL. Aerosol delivery of diethylenetriamine/nitric oxide, a nitric oxide adduct, causes selective pulmonary vasodilation in perinatal lambs. J Lab Clin Med 134: 419-425, 1999.

72. Cornfield DN, Reeve HL, Tolarova S, Weir EK, and Archer S. Oxygen causes fetal pulmonary vasodilation through activation of a calcium-dependent potassium channel. Proc Natl Acad Sci U S A 93: 8089-8094, 1996.

73. Courboulin A, Tremblay VL, Barrier M, Meloche J, Jacob MH, Chapolard M, Bisserier M, Paulin R, Lambert C, Provencher S, and Bonnet S. Kruppel-like factor 5 contributes to pulmonary artery smooth muscle proliferation and resistance to apoptosis in human pulmonary arterial hypertension. Respir Res 12: 128, 2011.

74. Cribbs JT, and Strack S. Functional characterization of phosphorylation sites in dynamin-related protein 1. Methods in enzymology 457: 231-253, 2009.

75. Cribbs JT, and Strack S. Reversible phosphorylation of Drp1 by cyclic AMPdependent protein kinase and calcineurin regulates mitochondrial fission and cell death. EMBO Rep 8: 939-944, 2007.

76. Crossno JT, Jr., Garat CV, Reusch JE, Morris KG, Dempsey EC, McMurtry IF, Stenmark KR, and Klemm DJ. Rosiglitazone attenuates hypoxia-induced pulmonary arterial remodeling. American journal of physiology Lung cellular and molecular physiology 292: L885-897, 2007.

77. Cullen JJ, Weydert C, Hinkhouse MM, Ritchie J, Domann FE, Spitz D, and Oberley LW. The role of manganese superoxide dismutase in the growth of pancreatic adenocarcinoma. Cancer Res 63: 1297-1303, 2003.

78. Darnell JE, Jr. STATs and gene regulation. Science 277: 1630-1635, 1997.

79. Dasgupta A, Chen KH, Munk RB, Sasaki CY, Curtis J, Longo DL, and Ghosh P. Mechanism of Activation-Induced Downregulation of Mitofusin 2 in Human Peripheral Blood T Cells. J Immunol 195: 5780-5786, 2015.

80. de Brito OM, and Scorrano L. Mitofusin 2 tethers endoplasmic reticulum to mitochondria. Nature 456: 605-610, 2008.

81. Delettre C, Lenaers G, Griffoin JM, Gigarel N, Lorenzo C, Belenguer P, Pelloquin L, Grosgeorge J, Turc-Carel C, Perret E, Astarie-Dequeker C, Lasquellec L, Arnaud B, Ducommun B, Kaplan J, and Hamel CP. Nuclear gene OPA1, encoding a mitochondrial dynamin-related protein, is mutated in dominant optic atrophy. Nat Genet 26: 207-210, 2000 .

82. Denton RM, Randle PJ, and Martin BR. Stimulation by calcium ions of pyruvate dehydrogenase phosphate phosphatase. Biochem J 128: 161-163, 1972.

83. Diebold I, Hennigs JK, Miyagawa K, Li CG, Nickel NP, Kaschwich M, Cao A, Wang L, Reddy S, Chen PI, Nakahira K, Alcazar MA, Hopper RK, Ji L, Feldman BJ, and Rabinovitch M. BMPR2 preserves mitochondrial function and DNA during reoxygenation to promote endothelial cell survival and reverse pulmonary hypertension. Cell Metab 21: 
596-608, 2015.

84. Dromparis P, Paulin R, Sutendra G, Qi AC, Bonnet S, and Michelakis ED.

Uncoupling protein 2 deficiency mimics the effects of hypoxia and endoplasmic reticulum stress on mitochondria and triggers pseudohypoxic pulmonary vascular remodeling and pulmonary hypertension. Circ Res 113: 126-136, 2013.

85. Dunham-Snary KJ, Wu D, Potus F, Sykes EA, Mewburn JD, Charles RL, Eaton P, Sultanian RA, and Archer SL. Ndufs2, a Core Subunit of Mitochondrial Complex I, Is Essential for Acute Oxygen-Sensing and Hypoxic Pulmonary Vasoconstriction. Circ Res 124: 1727-1746, 2019.

86. Dunham-Snary KJ, Wu D, Potus F, Sykes EA, Mewburn JD, Charles RL, Eaton P, Sultanian RA, and Archer SL. Ndufs2, A Core Subunit of Mitochondrial Complex I, Is Essential for Acute Oxygen-Sensing and Hypoxic Pulmonary Vasoconstriction. Circulation research 2019.

87. Duprat F, Guillemare E, Romey G, Fink M, Lesage F, Lazdunski M, and Honore E. Susceptibility of cloned K+ channels to reactive oxygen species. Proc Natl Acad Sci U S A 92: 11796-11800, 1995.

88. Egnatchik RA, Brittain EL, Shah AT, Fares WH, Ford HJ, Monahan K, Kang CJ, Kocurek EG, Zhu S, Luong T, Nguyen TT, Hysinger E, Austin ED, Skala MC, Young JD, Roberts LJ, 2nd, Hemnes AR, West J, and Fessel JP. Dysfunctional BMPR2 signaling drives an abnormal endothelial requirement for glutamine in pulmonary arterial hypertension. Pulm Circ 7: 186-199, 2017.

89. Ehlken N, Lichtblau M, Klose H, Weidenhammer J, Fischer C, Nechwatal R, Uiker S, Halank M, Olsson K, Seeger W, Gall H, Rosenkranz S, Wilkens H, Mertens D, Seyfarth HJ, Opitz C, Ulrich S, Egenlauf B, and Grunig E. Exercise training improves peak oxygen consumption and haemodynamics in patients with severe pulmonary arterial hypertension and inoperable chronic thrombo-embolic pulmonary hypertension: a prospective, randomized, controlled trial. European heart journal 37: 35-44, 2016. 90. Enache I, Charles AL, Bouitbir J, Favret F, Zoll J, Metzger D, Oswald-Mammosser M, Geny B, and Charloux A. Skeletal muscle mitochondrial dysfunction precedes right ventricular impairment in experimental pulmonary hypertension. Mol Cell Biochem 373: 161-170, 2013.

91. Epstein AC, Gleadle JM, McNeill LA, Hewitson KS, O'Rourke J, Mole DR, Mukherji M, Metzen E, Wilson MI, Dhanda A, Tian YM, Masson N, Hamilton DL, Jaakkola P, Barstead R, Hodgkin J, Maxwell PH, Pugh CW, Schofield CJ, and Ratcliffe PJ. C. elegans EGL-9 and mammalian homologs define a family of dioxygenases that regulate HIF by prolyl hydroxylation. Cell 107: 43-54, 2001.

92. Esterbauer H, Schneitler C, Oberkofler H, Ebenbichler C, Paulweber B, Sandhofer F, Ladurner G, Hell E, Strosberg AD, Patsch JR, Krempler F, and Patsch W. A common polymorphism in the promoter of UCP2 is associated with decreased risk of obesity in middle-aged humans. Nat Genet 28: 178-183, 2001.

93. Euler USv, and Liljestrand G. Observations on the Pulmonary Arterial Blood Pressure in the Cat. Acta Physiologica Scandinavica 12: 301-320, 1946.

94. Fagan KA, Oka M, Bauer NR, Gebb SA, Ivy DD, Morris KG, and McMurtry IF. Attenuation of acute hypoxic pulmonary vasoconstriction and hypoxic pulmonary hypertension in mice by inhibition of Rho-kinase. American journal of physiology Lung cellular and molecular physiology 287: L656-664, 2004. 
95. Fang X, Chen X, Zhong G, Chen Q, and Hu C. Mitofusin 2 Downregulation Triggers Pulmonary Artery Smooth Muscle Cell Proliferation and Apoptosis Imbalance in Rats With Hypoxic Pulmonary Hypertension Via the PI3K/Akt and Mitochondrial Apoptosis Pathways. J Cardiovasc Pharmacol 67: 164-174, 2016.

96. Fang YH, Piao L, Hong Z, Toth PT, Marsboom G, Bache-Wiig P, Rehman J, and Archer SL. Therapeutic inhibition of fatty acid oxidation in right ventricular hypertrophy: exploiting Randle's cycle. J Mol Med (Berl) 90: 31-43, 2012.

97. Fernandez-Aguera MC, Gao L, Gonzalez-Rodriguez P, Pintado CO, AriasMayenco I, Garcia-Flores P, Garcia-Perganeda A, Pascual A, Ortega-Saenz P, and Lopez-Barneo J. Oxygen Sensing by Arterial Chemoreceptors Depends on Mitochondrial Complex I Signaling. Cell Metab 22: 825-837, 2015.

98. Fijalkowska I, Xu W, Comhair SA, Janocha AJ, Mavrakis LA, Krishnamachary B, Zhen L, Mao T, Richter A, Erzurum SC, and Tuder RM. Hypoxia inducible-factor1alpha regulates the metabolic shift of pulmonary hypertensive endothelial cells. Am J Pathol 176: 1130-1138, 2010.

99. Filadi R, Greotti E, Turacchio G, Luini A, Pozzan T, and Pizzo P. Mitofusin 2 ablation increases endoplasmic reticulum-mitochondria coupling. Proc Natl Acad Sci U S A 112: E2174-2181, 2015.

100. Filipowicz W, Bhattacharyya SN, and Sonenberg N. Mechanisms of posttranscriptional regulation by microRNAs: are the answers in sight? Nat Rev Genet 9: 102114, 2008.

101. Firth AL, Remillard CV, Platoshyn O, Fantozzi I, Ko EA, and Yuan JX. Functional ion channels in human pulmonary artery smooth muscle cells: Voltage-dependent cation channels. Pulmonary circulation 1: 48-71, 2011.

102. Foshat $\mathbf{M}$, and Boroumand $\mathbf{N}$. The Evolving Classification of Pulmonary Hypertension. Archives of pathology \& laboratory medicine 141: 696-703, 2017.

103. Foskett JK, and Philipson B. The mitochondrial $\mathrm{Ca}(2+)$ uniporter complex. J Mol Cell Cardiol 78: 3-8, 2015.

104. Friedman JR, Lackner LL, West M, DiBenedetto JR, Nunnari J, and Voeltz GK. ER tubules mark sites of mitochondrial division. Science 334: 358-362, 2011.

105. Frise MC, Cheng HY, Nickol AH, Curtis MK, Pollard KA, Roberts DJ, Ratcliffe PJ, Dorrington KL, and Robbins PA. Clinical iron deficiency disturbs normal human responses to hypoxia. J Clin Invest 126: 2139-2150, 2016.

106. Gabaldon T, and Huynen MA. Prediction of protein function and pathways in the genome era. Cell Mol Life Sci 61: 930-944, 2004.

107. Gardener MJ, Johnson IT, Burnham MP, Edwards G, Heagerty AM, and Weston AH. Functional evidence of a role for two-pore domain potassium channels in rat mesenteric and pulmonary arteries. Br J Pharmacol 142: 192-202, 2004.

108. Gatenby RA, and Gillies RJ. Why do cancers have high aerobic glycolysis? Nat Rev Cancer 4: 891-899, 2004.

109. Gkikas I, Palikaras K, and Tavernarakis N. The Role of Mitophagy in Innate Immunity. Front Immunol 9: 1283, 2018.

110. Gomberg-Maitland M, Schilz R, Mediratta A, Addetia K, Coslet S, Thomeas V, Gillies H, and Oudiz RJ. Phase I Safety Study of Ranolazine in Pulmonary Arterial Hypertension. Pulmonary Circulation 5: 691-700, 2015.

111. Gomez-Arroyo J, Mizuno S, Szczepanek K, Van Tassell B, Natarajan R, dos 
Remedios CG, Drake JI, Farkas L, Kraskauskas D, Wijesinghe DS, Chalfant CE, Bigbee J, Abbate A, Lesnefsky EJ, Bogaard HJ, and Voelkel NF. Metabolic gene remodeling and mitochondrial dysfunction in failing right ventricular hypertrophy secondary to pulmonary arterial hypertension. Circ Heart Fail 6: 136-144, 2013.

112. Gong M, Fragakis N, Zhang C, Zhang Z, Li G, and Liu T. Ranolazine as a novel therapy for pulmonary arterial hypertension. International journal of cardiology 223: 860862, 2016.

113. Graham BB, Kumar R, Mickael C, Sanders L, Gebreab L, Huber KM, Perez M, Smith-Jones P, Serkova NJ, and Tuder RM. Severe pulmonary hypertension is associated with altered right ventricle metabolic substrate uptake. Am J Physiol Lung Cell Mol Physiol 309: L435-440, 2015.

114. Grant JL, Naylor RW, and Crandell WB. Bronchial adenoma resection with relief of hypoxic pulmonary vasoconstriction. Chest 77: 446-449, 1980.

115. Gropler RJ, Siegel BA, Lee KJ, Moerlein SM, Perry DJ, Bergmann SR, and Geltman EM. Nonuniformity in myocardial accumulation of fluorine-18fluorodeoxyglucose in normal fasted humans. J Nucl Med 31: 1749-1756, 1990. 116. Grunig E, Ehlken N, Ghofrani A, Staehler G, Meyer FJ, Juenger J, Opitz CF, Klose H, Wilkens H, Rosenkranz S, Olschewski H, and Halank M. Effect of exercise and respiratory training on clinical progression and survival in patients with severe chronic pulmonary hypertension. Respiration; international review of thoracic diseases 81: 394-401, 2011.

117. Grunig E, Lichtblau M, Ehlken N, Ghofrani HA, Reichenberger F, Staehler G, Halank M, Fischer C, Seyfarth HJ, Klose H, Meyer A, Sorichter S, Wilkens H, Rosenkranz S, Opitz C, Leuchte H, Karger G, Speich R, and Nagel C. Safety and efficacy of exercise training in various forms of pulmonary hypertension. Eur Respir J 40: 84-92, 2012. 118. Grunig E, Maier F, Ehlken N, Fischer C, Lichtblau M, Blank N, Fiehn C, Stockl F, Prange F, Staehler G, Reichenberger F, Tiede H, Halank M, Seyfarth HJ, Wagner S, and Nagel C. Exercise training in pulmonary arterial hypertension associated with connective tissue diseases. Arthritis research \& therapy 14: R148, 2012.

119. Guazzi M, Gomberg-Maitland M, and Arena R. Pulmonary hypertension in heart failure with preserved ejection fraction. The Journal of heart and lung transplantation : the official publication of the International Society for Heart Transplantation 34: 273-281, 2015. 120. Guedes-Dias P, de Proenca J, Soares TR, Leitao-Rocha A, Pinho BR, Duchen MR, and Oliveira JM. HDAC6 inhibition induces mitochondrial fusion, autophagic flux and reduces diffuse mutant huntingtin in striatal neurons. Biochim Biophys Acta 1852: 24842493, 2015.

121. Guo X, Chen KH, Guo Y, Liao H, Tang J, and Xiao RP. Mitofusin 2 triggers vascular smooth muscle cell apoptosis via mitochondrial death pathway. Circ Res 101: 1113-1122, 2007.

122. Gurney AM, Osipenko ON, MacMillan D, McFarlane KM, Tate RJ, and Kempsill FE. Two-pore domain K channel, TASK-1, in pulmonary artery smooth muscle cells. Circ Res 93: 957-964, 2003.

123. Guzy RD, Hoyos B, Robin E, Chen H, Liu L, Mansfield KD, Simon MC, Hammerling $\mathbf{U}$, and Schumacker PT. Mitochondrial complex III is required for hypoxiainduced ROS production and cellular oxygen sensing. Cell Metab 1: 401-408, 2005.

124. Hagan G, Southwood M, Treacy C, Ross RM, Soon E, Coulson J, Sheares K, 
Screaton N, Pepke-Zaba J, Morrell NW, and Rudd JH. (18)FDG PET imaging can quantify increased cellular metabolism in pulmonary arterial hypertension: A proof-of-principle study. Pulm Circ 1: 448-455, 2011.

125. Hales CA. Physiological Function of Hypoxic Pulmonary Vasoconstriction. In: Hypoxic Pulmonary Vasoconstriction Developments in Cardiovascular Medicine, edited by Yuan JXJ. Boston, MA: Springer, 2004, p. 3-14.

126. Hall AR, Burke N, Dongworth RK, and Hausenloy DJ. Mitochondrial fusion and fission proteins: novel therapeutic targets for combating cardiovascular disease. $\mathrm{BrJ}$ Pharmacol 171: 1890-1906, 2014.

127. Handschin C, and Spiegelman BM. Peroxisome proliferator-activated receptor gamma coactivator 1 coactivators, energy homeostasis, and metabolism. Endocr Rev 27: 728-735, 2006.

128. Haslip M, Dostanic I, Huang Y, Zhang Y, Russell KS, Jurczak MJ, Mannam P, Giordano F, Erzurum SC, and Lee PJ. Endothelial uncoupling protein 2 regulates mitophagy and pulmonary hypertension during intermittent hypoxia. Arterioscler Thromb Vasc Biol 35: 1166-1178, 2015.

129. Hasunuma K, Rodman DM, and McMurtry IF. Effects of K+ channel blockers on vascular tone in the perfused rat lung. The American review of respiratory disease 144: 884887, 1991.

130. Hayes GB, and Oudiz RJ. Pulmonary Hypertension: A Patient's Survival Guide. Pulmonary Hypertension Association, 2004.

131. Head B, Griparic L, Amiri M, Gandre-Babbe S, and van der Bliek AM. Inducible proteolytic inactivation of OPA1 mediated by the OMA1 protease in mammalian cells. J Cell Biol 187: 959-966, 2009.

132. Hemnes AR, Brittain EL, Trammell AW, Fessel JP, Austin ED, Penner N, Maynard KB, Gleaves L, Talati M, Absi T, Disalvo T, and West J. Evidence for right ventricular lipotoxicity in heritable pulmonary arterial hypertension. American journal of respiratory and critical care medicine 189: 325-334, 2014.

133. Henrohn D, Bjorkstrand K, Lundberg JO, Granstam SO, Baron T, Ingimarsdottir IJ, Hedenstrom H, Malinovschi A, Wernroth ML, Jansson M, Hedeland M, and Wikstrom G. Effects of Oral Supplementation With Nitrate-Rich Beetroot Juice in Patients With Pulmonary Arterial Hypertension-Results From BEET-PAH, an Exploratory Randomized, Double-Blind, Placebo-Controlled, Crossover Study. Journal of cardiac failure 24: 640-653, 2018.

134. Henry TD, Archer SL, Nelson D, Weir EK, and From AH. Postischemic oxygen radical production varies with duration of ischemia. Am J Physiol 264: H1478-1484, 1993. 135. Herr DJ, Baarine M, Aune SE, Li X, Ball LE, Lemasters JJ, Beeson CC, Chou JC, and Menick DR. HDAC1 localizes to the mitochondria of cardiac myocytes and contributes to early cardiac reperfusion injury. Journal of molecular and cellular cardiology 114: 309-319, 2018.

136. Hindmarch CCT, and Murphy D. The transcriptome and the hypothalamoneurohypophyseal system. Endocr Dev 17: 1-10, 2010.

137. Hirschey MD, Shimazu T, Jing E, Grueter CA, Collins AM, Aouizerat B, Stancakova A, Goetzman E, Lam MM, Schwer B, Stevens RD, Muehlbauer MJ, Kakar S, Bass NM, Kuusisto J, Laakso M, Alt FW, Newgard CB, Farese RV, Jr., Kahn CR, and Verdin E. SIRT3 deficiency and mitochondrial protein hyperacetylation accelerate the 
development of the metabolic syndrome. Molecular cell 44: 177-190, 2011.

138. Hodge DR, Xiao W, Peng B, Cherry JC, Munroe DJ, and Farrar WL. Enforced expression of superoxide dismutase 2 /manganese superoxide dismutase disrupts autocrine interleukin- 6 stimulation in human multiple myeloma cells and enhances dexamethasone-induced apoptosis. Cancer Res 65: 6255-6263, 2005.

139. Holness MJ, and Sugden MC. Regulation of pyruvate dehydrogenase complex activity by reversible phosphorylation. Biochem Soc Trans 31: 1143-1151, 2003.

140. Hong Z, Chen KH, DasGupta A, Potus F, Dunham-Snary K, Bonnet S, Tian L, Fu J, Breuils-Bonnet S, Provencher S, Wu D, Mewburn J, Ormiston ML, and Archer SL. MicroRNA-138 and MicroRNA-25 Down-regulate Mitochondrial Calcium Uniporter, Causing the Pulmonary Arterial Hypertension Cancer Phenotype. American journal of respiratory and critical care medicine 195: 515-529, 2017.

141. Hoppins S, Edlich F, Cleland MM, Banerjee S, McCaffery JM, Youle RJ, and Nunnari J. The soluble form of Bax regulates mitochondrial fusion via MFN2 homotypic complexes. Molecular cell 41: 150-160, 2011.

142. Humbert M, Lau EM, Montani D, Jais X, Sitbon O, and Simonneau G. Advances in therapeutic interventions for patients with pulmonary arterial hypertension. Circulation 130: 2189-2208, 2014.

143. Hurt EM, Thomas SB, Peng B, and Farrar WL. Molecular consequences of SOD2 expression in epigenetically silenced pancreatic carcinoma cell lines. British journal of cancer 97: 1116-1123, 2007.

144. Ishihara N, Eura Y, and Mihara K. Mitofusin 1 and 2 play distinct roles in mitochondrial fusion reactions via GTPase activity. J Cell Sci 117: 6535-6546, 2004.

145. Ivan M, Kondo K, Yang H, Kim W, Valiando J, Ohh M, Salic A, Asara JM, Lane WS, and Kaelin WG, Jr. HIFalpha targeted for VHL-mediated destruction by proline hydroxylation: implications for 02 sensing. Science 292: 464-468, 2001.

146. Iwai K, Yamanaka K, Kamura T, Minato N, Conaway RC, Conaway JW, Klausner RD, and Pause A. Identification of the von Hippel-lindau tumor-suppressor protein as part of an active E3 ubiquitin ligase complex. Proc Natl Acad Sci U S A 96: 12436-12441, 1999.

147. Jaakkola P, Mole DR, Tian YM, Wilson MI, Gielbert J, Gaskell SJ, von Kriegsheim A, Hebestreit HF, Mukherji M, Schofield CJ, Maxwell PH, Pugh CW, and Ratcliffe PJ. Targeting of HIF-alpha to the von Hippel-Lindau ubiquitylation complex by 02-regulated prolyl hydroxylation. Science 292: 468-472, 2001.

148. Jafri S, Sivasothy P, Wells F, and Morrell NW. Clinical demonstration of efficiency and reversibility of hypoxic pulmonary vasoconstriction in a patient presenting with unilateral incomplete bronchial occlusion. Pulm Circ 1: 119-121, 2011.

149. Jensen KS, Micco AJ, Czartolomna J, Latham L, and Voelkel NF. Rapid onset of hypoxic vasoconstriction in isolated lungs. J Appl Physiol (1985) 72: 2018-2023, 1992. 150. Jin B, Li Y, and Robertson KD. DNA methylation: superior or subordinate in the epigenetic hierarchy? Genes Cancer 2: 607-617, 2011.

151. John B. West RBS, Andrew M. Luks, James S. Milledge. High Altitude Medicine and Physiology. CRC Press, 2012.

152. Kabitz HJ, Bremer HC, Schwoerer A, Sonntag F, Walterspacher S, Walker DJ, Ehlken N, Staehler G, Windisch W, and Grunig E. The combination of exercise and respiratory training improves respiratory muscle function in pulmonary hypertension. Lung 192: 321-328, 2014. 
153. Kamerkar SC, Kraus F, Sharpe AJ, Pucadyil TJ, and Ryan MT. Dynamin-related protein 1 has membrane constricting and severing abilities sufficient for mitochondrial and peroxisomal fission. Nat Commun 9: 5239, 2018.

154. Khan SS, Cuttica MJ, Beussink-Nelson L, Kozyleva A, Sanchez C, Mkrdichian H, Selvaraj S, Dematte JE, Lee DC, and Shah SJ. Effects of ranolazine on exercise capacity, right ventricular indices, and hemodynamic characteristics in pulmonary arterial hypertension: a pilot study. Pulm Circ 5: 547-556, 2015.

155. Kim DH, Kim HS, Choi CW, Kim EK, Kim BI, and Choi JH. Risk factors for pulmonary artery hypertension in preterm infants with moderate or severe bronchopulmonary dysplasia. Neonatology 101: 40-46, 2012.

156. Kim EK, Lee JH, Oh YM, Lee YS, and Lee SD. Rosiglitazone attenuates hypoxiainduced pulmonary arterial hypertension in rats. Respirology 15: 659-668, 2010.

157. Kim GH, Ryan JJ, Marsboom G, and Archer SL. Epigenetic mechanisms of pulmonary hypertension. Pulm Circ 1: 347-356, 2011.

158. Kirichok Y, Krapivinsky G, and Clapham DE. The mitochondrial calcium uniporter is a highly selective ion channel. Nature 427: 360-364, 2004.

159. Koshiba T, Detmer SA, Kaiser JT, Chen H, McCaffery JM, and Chan DC. Structural basis of mitochondrial tethering by mitofusin complexes. Science 305: 858-862, 2004.

160. Kovacs L, Cao Y, Han W, Meadows L, Kovacs-Kasa A, Kondrikov D, Verin AD, Barman SA, Dong Z, Huo Y, and Su Y. PFKFB3 in Smooth Muscle Promotes Vascular Remodeling in Pulmonary Arterial Hypertension. American journal of respiratory and critical care medicine 2019.

161. Kraus F, and Ryan MT. The constriction and scission machineries involved in mitochondrial fission. J Cell Sci 130: 2953-2960, 2017.

162. Kreitner KF. Noninvasive imaging of pulmonary hypertension. Semin Respir Crit Care Med 35: 99-111, 2014.

163. Kreymborg K, Uchida S, Gellert P, Schneider A, Boettger T, Voswinckel R, Wietelmann A, Szibor M, Weissmann N, Ghofrani AH, Schermuly R, Schranz D, Seeger $\mathbf{W}$, and Braun T. Identification of right heart-enriched genes in a murine model of chronic outflow tract obstruction. Journal of molecular and cellular cardiology 49: 598-605, 2010. 164. Kuhr FK, Smith KA, Song MY, Levitan I, and Yuan JX. New mechanisms of pulmonary arterial hypertension: role of $\mathrm{Ca}(2)(+)$ signaling. Am J Physiol Heart Circ Physiol 302: H1546-1562, 2012.

165. Lane N, and Martin W. The energetics of genome complexity. Nature 467: 929-934, 2010 .

166. Leboucher GP, Tsai YC, Yang M, Shaw KC, Zhou M, Veenstra TD, Glickman MH, and Weissman AM. Stress-induced phosphorylation and proteasomal degradation of mitofusin 2 facilitates mitochondrial fragmentation and apoptosis. Molecular cell 47: 547$557,2012$.

167. Lee JE, Westrate LM, Wu H, Page C, and Voeltz GK. Multiple dynamin family members collaborate to drive mitochondrial division. Nature 540: 139-143, 2016.

168. Lee JY, Kapur M, Li M, Choi MC, Choi S, Kim HJ, Kim I, Lee E, Taylor JP, and Yao TP. MFN1 deacetylation activates adaptive mitochondrial fusion and protects metabolically challenged mitochondria. J Cell Sci 127: 4954-4963, 2014.

169. Lee R, Feinbaum R, and Ambros V. A short history of a short RNA. Cell 116: S8992, 81 p following S96, 2004. 
170. Lee YJ, Jeong SY, Karbowski M, Smith CL, and Youle RJ. Roles of the mammalian mitochondrial fission and fusion mediators Fis1, Drp1, and Opa1 in apoptosis. Mol Biol Cell 15: 5001-5011, 2004.

171. Legchenko E, Chouvarine P, Borchert P, Fernandez-Gonzalez A, Snay E, Meier M, Maegel L, Mitsialis SA, Rog-Zielinska EA, Kourembanas S, Jonigk D, and Hansmann G. PPARgamma agonist pioglitazone reverses pulmonary hypertension and prevents right heart failure via fatty acid oxidation. Sci Transl Med 10: 2018.

172. Li J, Donath S, Li Y, Qin D, Prabhakar BS, and Li P. miR-30 regulates mitochondrial fission through targeting p53 and the dynamin-related protein-1 pathway. PLoS Genet 6: e1000795, 2010.

173. Li M, Riddle S, Zhang H, D'Alessandro A, Flockton A, Serkova NJ, Hansen KC, Moldvan R, McKeon BA, Frid M, Kumar S, Li H, Liu H, Caanovas A, Medrano JF, Thomas MG, Iloska D, Plecita-Hlavata L, Jezek P, Pullamsetti S, Fini MA, El Kasmi KC, Zhang Q, and Stenmark KR. Metabolic Reprogramming Regulates the Proliferative and Inflammatory Phenotype of Adventitial Fibroblasts in Pulmonary Hypertension Through the Transcriptional Corepressor C-Terminal Binding Protein-1. Circulation 134: 1105-1121, 2016.

174. Li M, Riddle SR, Frid MG, El Kasmi KC, McKinsey TA, Sokol RJ, Strassheim D, Meyrick B, Yeager ME, Flockton AR, McKeon BA, Lemon DD, Horn TR, Anwar A, Barajas C, and Stenmark KR. Emergence of fibroblasts with a proinflammatory epigenetically altered phenotype in severe hypoxic pulmonary hypertension. Journal of immunology (Baltimore, Md: 1950) 187: 2711-2722, 2011.

175. Liang $\mathbf{H}$, and Ward WF. PGC-1alpha: a key regulator of energy metabolism. $A d v$ Physiol Educ 30: 145-151, 2006.

176. Liberti MV, and Locasale JW. The Warburg Effect: How Does it Benefit Cancer Cells? Trends Biochem Sci 41: 211-218, 2016.

177. Light RB. Pulmonary pathophysiology of pneumococcal pneumonia. Semin Respir Infect 14: 218-226, 1999.

178. Liles JT, Hoyer K, Oliver J, Chi L, Dhalla AK, and Belardinelli L. Ranolazine reduces remodeling of the right ventricle and provoked arrhythmias in rats with pulmonary hypertension.J Pharmacol Exp Ther 353: 480-489, 2015.

179. Lkhagva B, Kao YH, Lee TI, Lee TW, Cheng WL, and Chen YJ. Activation of Class I histone deacetylases contributes to mitochondrial dysfunction in cardiomyocytes with altered complex activities. Epigenetics 13: 376-385, 2018.

180. Loson OC, Liu R, Rome ME, Meng S, Kaiser JT, Shan SO, and Chan DC. The mitochondrial fission receptor MiD51 requires ADP as a cofactor. Structure 22: 367-377, 2014.

181. Loson OC, Meng S, Ngo H, Liu R, Kaiser JT, and Chan DC. Crystal structure and functional analysis of MiD49, a receptor for the mitochondrial fission protein Drp1. Protein Sci 24: 386-394, 2015.

182. Loson OC, Song Z, Chen H, and Chan DC. Fis1, Mff, MiD49, and MiD51 mediate Drp1 recruitment in mitochondrial fission. Mol Biol Cell 24: 659-667, 2013.

183. Lu X, Bijli KM, Ramirez A, Murphy TC, Kleinhenz J, and Hart CM. Hypoxia downregulates PPARgamma via an ERK1/2-NF-kappaB-Nox4-dependent mechanism in human pulmonary artery smooth muscle cells. Free Radic Biol Med 63: 151-160, 2013. 184. Lu Z, Li S, Zhao S, and Fa X. Upregulated miR-17 Regulates Hypoxia-Mediated 
Human Pulmonary Artery Smooth Muscle Cell Proliferation and Apoptosis by Targeting Mitofusin 2. Med Sci Monit 22: 3301-3308, 2016.

185. Ma C, Zhang C, Ma M, Zhang L, Zhang L, Zhang F, Chen Y, Cao F, Li M, Wang G, Shen T, Yao H, Liu Y, Pan Z, Song S, and Zhu D. MiR-125a regulates mitochondrial homeostasis through targeting mitofusin 1 to control hypoxic pulmonary vascular remodeling. J Mol Med (Berl) 95: 977-993, 2017.

186. Madden JA, Dawson CA, and Harder DR. Hypoxia-induced activation in small isolated pulmonary arteries from the cat. J Appl Physiol (1985) 59: 113-118, 1985.

187. Madden JA, Vadula MS, and Kurup VP. Effects of hypoxia and other vasoactive agents on pulmonary and cerebral artery smooth muscle cells. Am J Physiol 263: L384-393, 1992.

188. Malka F, Guillery O, Cifuentes-Diaz C, Guillou E, Belenguer P, Lombes A, and Rojo M. Separate fusion of outer and inner mitochondrial membranes. EMBO Rep 6: 853859, 2005.

189. Manalo DJ, Rowan A, Lavoie T, Natarajan L, Kelly BD, Ye SQ, Garcia JG, and Semenza GL. Transcriptional regulation of vascular endothelial cell responses to hypoxia by HIF-1. Blood 105: 659-669, 2005.

190. Mao K, and Klionsky DJ. Participation of mitochondrial fission during mitophagy. Cell cycle 12: 3131-3132, 2013.

191. Marchi S, Lupini L, Patergnani S, Rimessi A, Missiroli S, Bonora M, Bononi A, Corra F, Giorgi C, De Marchi E, Poletti F, Gafa R, Lanza G, Negrini M, Rizzuto R, and Pinton P. Downregulation of the mitochondrial calcium uniporter by cancer-related miR25. Curr Biol 23: 58-63, 2013.

192. Marsboom G, Toth PT, Ryan JJ, Hong Z, Wu X, Fang YH, Thenappan T, Piao L, Zhang HJ, Pogoriler J, Chen Y, Morrow E, Weir EK, Rehman J, and Archer SL. Dynaminrelated protein 1-mediated mitochondrial mitotic fission permits hyperproliferation of vascular smooth muscle cells and offers a novel therapeutic target in pulmonary hypertension. Circ Res 110: 1484-1497, 2012.

193. Marsboom G, Wietholt C, Haney CR, Toth PT, Ryan JJ, Morrow E, Thenappan T, Bache-Wiig P, Piao L, Paul J, Chen CT, and Archer SL. Lung (1)(8)F-fluorodeoxyglucose positron emission tomography for diagnosis and monitoring of pulmonary arterial hypertension. American journal of respiratory and critical care medicine 185: 670-679, 2012.

194. Masson N, Willam C, Maxwell PH, Pugh CW, and Ratcliffe PJ. Independent function of two destruction domains in hypoxia-inducible factor-alpha chains activated by prolyl hydroxylation. EMBO J 20: 5197-5206, 2001.

195. Matoulkova E, Michalova E, Vojtesek B, and Hrstka R. The role of the 3' untranslated region in post-transcriptional regulation of protein expression in mammalian cells. RNA Biol 9: 563-576, 2012.

196. Matsunaga K, Yanagawa M, Otsuka T, Hirata H, Kijima T, Kumanogoh A, Tomiyama N, Shimosegawa E, and Hatazawa J. Quantitative pulmonary blood flow measurement using (15)O-H2O PET with and without tissue fraction correction: a comparison study. EJNMMI Res 7: 102, 2017.

197. Matsushita T, Ikeda S, Miyahara Y, Yakabe K, Yamaguchi K, Furukawa K, Iwasaki T, Shikuwa M, Fukui J, and Kohno S. Use of [123I]-BMIPP myocardial scintigraphy for the clinical evaluation of a fatty-acid metabolism disorder of the right 
ventricle in chronic respiratory and pulmonary vascular disease. J Int Med Res 28: 111-123, 2000.

198. Mauban JR, Remillard CV, and Yuan JX. Hypoxic pulmonary vasoconstriction: role of ion channels. J Appl Physiol (1985) 98: 415-420, 2005.

199. Mazurek R, Dave JM, Chandran RR, Misra A, Sheikh AQ, and Greif DM. Vascular Cells in Blood Vessel Wall Development and Disease. Advances in pharmacology (San Diego, Calif) 78: 323-350, 2017.

200. McMurtry IF. BAY K 8644 potentiates and A23187 inhibits hypoxic vasoconstriction in rat lungs. Am J Physiol 249: H741-746, 1985.

201. McMurtry IF, Davidson AB, Reeves JT, and Grover RF. Inhibition of hypoxic pulmonary vasoconstriction by calcium antagonists in isolated rat lungs. Circ Res 38: 99104, 1976.

202. McMurtry MS, Bonnet S, Wu X, Dyck JR, Haromy A, Hashimoto K, and Michelakis ED. Dichloroacetate prevents and reverses pulmonary hypertension by inducing pulmonary artery smooth muscle cell apoptosis. Circ Res 95: 830-840, 2004. 203. Meloche J, Potus F, Vaillancourt M, Bourgeois A, Johnson I, Deschamps L, Chabot S, Ruffenach G, Henry S, Breuils-Bonnet S, Tremblay E, Nadeau V, Lambert C, Paradis R, Provencher S, and Bonnet S. Bromodomain-Containing Protein 4: The Epigenetic Origin of Pulmonary Arterial Hypertension. Circ Res 117: 525-535, 2015. 204. Mereles D, Ehlken N, Kreuscher S, Ghofrani S, Hoeper MM, Halank M, Meyer FJ, Karger G, Buss J, Juenger J, Holzapfel N, Opitz C, Winkler J, Herth FF, Wilkens H, Katus HA, Olschewski H, and Grunig E. Exercise and respiratory training improve exercise capacity and quality of life in patients with severe chronic pulmonary hypertension. Circulation 114: 1482-1489, 2006.

205. Michelakis ED, Gurtu V, Webster L, Barnes G, Watson G, Howard L, Cupitt J, Paterson I, Thompson RB, Chow K, O'Regan DP, Zhao L, Wharton J, Kiely DG, Kinnaird A, Boukouris AE, White C, Nagendran J, Freed DH, Wort SJ, Gibbs JSR, and Wilkins MR. Inhibition of pyruvate dehydrogenase kinase improves pulmonary arterial hypertension in genetically susceptible patients. Sci Transl Med 9: 2017.

206. Michelakis ED, Gurtu V, Webster L, Barnes G, Watson G, Howard L, Cupitt J, Paterson I, Thompson RB, Chow K, O'Regan DP, Zhao L, Wharton J, Kiely DG, Kinnaird A, Boukouris AE, White C, Nagendran J, Freed DH, Wort SJ, Gibbs JSR, and Wilkins MR. Inhibition of pyruvate dehydrogenase kinase improves pulmonary arterial hypertension in genetically susceptible patients. Science Translational Medicine 9: eaao4583, 2017.

207. Michelakis ED, Hampl V, Nsair A, Wu X, Harry G, Haromy A, Gurtu R, and Archer SL. Diversity in mitochondrial function explains differences in vascular oxygen sensing. Circ Res 90: 1307-1315, 2002.

208. Michelakis ED, McMurtry MS, Wu X-C, Dyck JRB, Moudgil R, Hopkins TA, Lopaschuk GD, Puttagunta L, Waite R, and Archer SL. Dichloroacetate, a Metabolic Modulator, Prevents and Reverses Chronic Hypoxic Pulmonary Hypertension in Rats. Circulation 105: 244-250, 2002.

209. Michelakis ED, Rebeyka I, Wu X, Nsair A, Thebaud B, Hashimoto K, Dyck JR, Haromy A, Harry G, Barr A, and Archer SL. 02 sensing in the human ductus arteriosus: regulation of voltage-gated $\mathrm{K}+$ channels in smooth muscle cells by a mitochondrial redox sensor. Circ Res 91: 478-486, 2002.

210. Michelakis ED, Reeve HL, Huang JM, Tolarova S, Nelson DP, Weir EK, and 
Archer SL. Potassium channel diversity in vascular smooth muscle cells. Can J Physiol Pharmacol 75: 889-897, 1997.

211. Michelakis ED, Thebaud B, Weir EK, and Archer SL. Hypoxic pulmonary vasoconstriction: redox regulation of 02 -sensitive $\mathrm{K}+$ channels by a mitochondrial 02sensor in resistance artery smooth muscle cells. Journal of molecular and cellular cardiology 37: 1119-1136, 2004.

212. Mick GJ, Wang X, Ling Fu C, and McCormick KL. Inhibition of leptin secretion by insulin and metformin in cultured rat adipose tissue. Biochim Biophys Acta 1502: 426-432, 2000.

213. Missiroli S, Patergnani S, Caroccia N, Pedriali G, Perrone M, Previati M, Wieckowski MR, and Giorgi C. Mitochondria-associated membranes (MAMs) and inflammation. Cell Death Dis 9: 329, 2018.

214. Mitchell P. Aspects of the chemiosmotic hypothesis. The Biochemical journal 116: 5P-6P, 1970.

215. Moudgil R, Michelakis ED, and Archer SL. Hypoxic pulmonary vasoconstriction. $J$ Appl Physiol (1985) 98: 390-403, 2005.

216. Murtaza G, Mermer P, Goldenberg A, Pfeil U, Paddenberg R, Weissmann N, Lochnit G, and Kummer W. TASK-1 potassium channel is not critically involved in mediating hypoxic pulmonary vasoconstriction of murine intra-pulmonary arteries. PloS one 12: e0174071, 2017.

217. Naeije R, and Brimioulle S. Physiology in medicine: importance of hypoxic pulmonary vasoconstriction in maintaining arterial oxygenation during acute respiratory failure. Critical care 5: 67-71, 2001.

218. Nagaya N, Goto Y, Satoh T, Uematsu M, Hamada S, Kuribayashi S, Okano Y, Kyotani S, Shimotsu Y, Fukuchi K, Nakanishi N, Takamiya M, and Ishida Y. Impaired regional fatty acid uptake and systolic dysfunction in hypertrophied right ventricle. J Nucl Med 39: 1676-1680, 1998.

219. Nagel C, Prange F, Guth S, Herb J, Ehlken N, Fischer C, Reichenberger F, Rosenkranz S, Seyfarth HJ, Mayer E, Halank M, and Grunig E. Exercise training improves exercise capacity and quality of life in patients with inoperable or residual chronic thromboembolic pulmonary hypertension. PloS one 7: e41603, 2012.

220. Nagendran J, Stewart K, Hoskinson M, and Archer SL. An anesthesiologist's guide to hypoxic pulmonary vasoconstriction: implications for managing single-lung anesthesia and atelectasis. Current opinion in anaesthesiology 19: 34-43, 2006.

221. Neuspiel M, Zunino R, Gangaraju S, Rippstein P, and McBride H. Activated mitofusin 2 signals mitochondrial fusion, interferes with Bax activation, and reduces susceptibility to radical induced depolarization. The Journal of biological chemistry 280: 25060-25070, 2005.

222. Niedermaier S, and Hilgendorff A. Bronchopulmonary dysplasia - an overview about pathophysiologic concepts. Mol Cell Pediatr 2: 2, 2015.

223. Nilius B, and Owsianik G. The transient receptor potential family of ion channels. Genome Biol 12: 218, 2011.

224. Nisbet RE, Bland JM, Kleinhenz DJ, Mitchell PO, Walp ER, Sutliff RL, and Hart

CM. Rosiglitazone attenuates chronic hypoxia-induced pulmonary hypertension in a mouse model. Am J Respir Cell Mol Biol 42: 482-490, 2010.

225. Ogata M. [Myocardial uptake of 125I-BMIPP in rats treated with adriamycin]. Kaku 
Igaku 26: 69-76, 1989.

226. Ohira H, deKemp R, Pena E, Davies RA, Stewart DJ, Chandy G, ContrerasDominguez V, Dennie C, Mc Ardle B, Mc Klein R, Renaud JM, DaSilva JN, Pugliese C, Dunne R, Beanlands R, and Mielniczuk LM. Shifts in myocardial fatty acid and glucose metabolism in pulmonary arterial hypertension: a potential mechanism for a maladaptive right ventricular response. Eur Heart J Cardiovasc Imaging 17: 1424-1431, 2016.

227. Oikawa M, Kagaya Y, Otani H, Sakuma M, Demachi J, Suzuki J, Takahashi T, Nawata J, Ido T, Watanabe J, and Shirato K. Increased [18F]fluorodeoxyglucose accumulation in right ventricular free wall in patients with pulmonary hypertension and the effect of epoprostenol. J Am Coll Cardiol 45: 1849-1855, 2005.

228. Olichon A, Baricault L, Gas N, Guillou E, Valette A, Belenguer P, and Lenaers G. Loss of OPA1 perturbates the mitochondrial inner membrane structure and integrity, leading to cytochrome c release and apoptosis. J Biol Chem 278: 7743-7746, 2003. 229. Olichon A, Elachouri G, Baricault L, Delettre C, Belenguer P, and Lenaers G. OPA1 alternate splicing uncouples an evolutionary conserved function in mitochondrial fusion from a vertebrate restricted function in apoptosis. Cell Death Differ 14: 682-692, 2007.

230. Olichon A, Emorine LJ, Descoins E, Pelloquin L, Brichese L, Gas N, Guillou E, Delettre C, Valette A, Hamel CP, Ducommun B, Lenaers G, and Belenguer P. The human dynamin-related protein OPA1 is anchored to the mitochondrial inner membrane facing the inter-membrane space. FEBS Lett 523: 171-176, 2002.

231. Otera $\mathbf{H}$, Ishihara $\mathbf{N}$, and Mihara K. New insights into the function and regulation of mitochondrial fission. Biochim Biophys Acta 1833: 1256-1268, 2013.

232. Otera H, Miyata N, Kuge $\mathbf{0}$, and Mihara K. Drp1-dependent mitochondrial fission via MiD49/51 is essential for apoptotic cristae remodeling. J Cell Biol 212: 531-544, 2016. 233. Otera H, Wang C, Cleland MM, Setoguchi K, Yokota S, Youle RJ, and Mihara K. Mff is an essential factor for mitochondrial recruitment of Drp1 during mitochondrial fission in mammalian cells. J Cell Biol 191: 1141-1158, 2010.

234. Padma $\mathbf{R}$, and Nagarajan $\mathbf{L}$. The human PIM-1 gene product is a protein serine kinase. Cancer Res 51: 2486-2489, 1991.

235. Paky A, Michael JR, Burke-Wolin TM, Wolin MS, and Gurtner GH. Endogenous production of superoxide by rabbit lungs: effects of hypoxia or metabolic inhibitors. J Appl Physiol (1985) 74: 2868-2874, 1993.

236. Palmer CS, Elgass KD, Parton RG, Osellame LD, Stojanovski D, and Ryan MT. Adaptor proteins MiD49 and MiD51 can act independently of Mff and Fis1 in Drp1 recruitment and are specific for mitochondrial fission. J Biol Chem 288: 27584-27593, 2013. 237. Palmer CS, Osellame LD, Laine D, Koutsopoulos OS, Frazier AE, and Ryan MT. MiD49 and MiD51, new components of the mitochondrial fission machinery. EMBO Rep 12: 565-573, 2011.

238. Pang B, Zheng XR, Tian JX, Gao TH, Gu GY, Zhang R, Fu YB, Pang Q, Li XG, and Liu Q. EZH2 promotes metabolic reprogramming in glioblastomas through epigenetic repression of EAF2-HIF1alpha signaling. Oncotarget 7: 45134-45143, 2016.

239. Park YY, Nguyen OT, Kang H, and Cho H. MARCH5-mediated quality control on acetylated Mfn1 facilitates mitochondrial homeostasis and cell survival. Cell Death Dis 5: e1172, 2014.

240. Patel MS, Nemeria NS, Furey W, and Jordan F. The pyruvate dehydrogenase 
complexes: structure-based function and regulation. J Biol Chem 289: 16615-16623, 2014.

241. Paulin R, Courboulin A, Meloche J, Mainguy V, Dumas de la Roque E, Saksouk N, Cote J, Provencher S, Sussman MA, and Bonnet S. Signal transducers and activators of transcription-3/pim1 axis plays a critical role in the pathogenesis of human pulmonary arterial hypertension. Circulation 123: 1205-1215, 2011.

242. Paulin R, Dromparis P, Sutendra G, Gurtu V, Zervopoulos S, Bowers L, Haromy A, Webster L, Provencher S, Bonnet S, and Michelakis ED. Sirtuin 3 deficiency is associated with inhibited mitochondrial function and pulmonary arterial hypertension in rodents and humans. Cell Metab 20: 827-839, 2014.

243. Perkovic V, Jardine MJ, Neal B, Bompoint S, Heerspink HJL, Charytan DM, Edwards R, Agarwal R, Bakris G, Bull S, Cannon CP, Capuano G, Chu P-L, de Zeeuw D, Greene T, Levin A, Pollock C, Wheeler DC, Yavin Y, Zhang H, Zinman B, Meininger G, Brenner BM, and Mahaffey KW. Canagliflozin and Renal Outcomes in Type 2 Diabetes and Nephropathy. New England Journal of Medicine 0: null.

244. Piao L, Fang YH, Cadete VJ, Wietholt C, Urboniene D, Toth PT, Marsboom G, Zhang HJ, Haber I, Rehman J, Lopaschuk GD, and Archer SL. The inhibition of pyruvate dehydrogenase kinase improves impaired cardiac function and electrical remodeling in two models of right ventricular hypertrophy: resuscitating the hibernating right ventricle. $J$ Mol Med (Berl) 88: 47-60, 2010.

245. Piao L, Fang YH, Parikh K, Ryan JJ, Toth PT, and Archer SL. Cardiac glutaminolysis: a maladaptive cancer metabolism pathway in the right ventricle in pulmonary hypertension. J Mol Med (Berl) 91: 1185-1197, 2013.

246. Plecita-Hlavata L, Tauber J, Li M, Zhang H, Flockton AR, Pullamsetti SS, Chelladurai P, D'Alessandro A, El Kasmi KC, Jezek P, and Stenmark KR. Constitutive Reprogramming of Fibroblast Mitochondrial Metabolism in Pulmonary Hypertension. Am J Respir Cell Mol Biol 55: 47-57, 2016.

247. Post JM, Hume JR, Archer SL, and Weir EK. Direct role for potassium channel inhibition in hypoxic pulmonary vasoconstriction. Am J Physiol 262: C882-890, 1992.

248. Potus F, Hindmarch CCT, Dunham-Snary KJ, Stafford J, and Archer SL.

Transcriptomic Signature of Right Ventricular Failure in Experimental Pulmonary Arterial Hypertension: Deep Sequencing Demonstrates Mitochondrial, Fibrotic, Inflammatory and Angiogenic Abnormalities. Int J Mol Sci 19: 2018.

249. Prakriya M, and Lewis RS. Store-Operated Calcium Channels. Physiological reviews 95: 1383-1436, 2015.

250. Prieto J, Leon M, Ponsoda X, Sendra R, Bort R, Ferrer-Lorente R, Raya A, LopezGarcia C, and Torres J. Early ERK1/2 activation promotes DRP1-dependent mitochondrial fission necessary for cell reprogramming. Nat Commun 7: 11124, 2016.

251. Puissegur MP, Mazure NM, Bertero T, Pradelli L, Grosso S, Robbe-Sermesant K, Maurin T, Lebrigand K, Cardinaud B, Hofman V, Fourre S, Magnone V, Ricci JE, Pouyssegur J, Gounon P, Hofman P, Barbry P, and Mari B. miR-210 is overexpressed in late stages of lung cancer and mediates mitochondrial alterations associated with modulation of HIF-1 activity. Cell Death Differ 18: 465-478, 2011.

252. Pullamsetti SS, Doebele C, Fischer A, Savai R, Kojonazarov B, Dahal BK, Ghofrani HA, Weissmann N, Grimminger F, Bonauer A, Seeger W, Zeiher AM, Dimmeler S, and Schermuly RT. Inhibition of microRNA-17 improves lung and heart function in experimental pulmonary hypertension. Am J Respir Crit Care Med 185: 409-419, 
2012.

253. Pyakurel A, Savoia C, Hess D, and Scorrano L. Extracellular regulated kinase phosphorylates mitofusin 1 to control mitochondrial morphology and apoptosis. Molecular cell 58: 244-254, 2015.

254. Qi X, Qvit N, Su YC, and Mochly-Rosen D. A novel Drp1 inhibitor diminishes aberrant mitochondrial fission and neurotoxicity. J Cell Sci 126: 789-802, 2013.

255. Rafikov R, Sun X, Rafikova O, Louise Meadows M, Desai AA, Khalpey Z, Yuan JX, Fineman JR, and Black SM. Complex I dysfunction underlies the glycolytic switch in pulmonary hypertensive smooth muscle cells. Redox Biol 6: 278-286, 2015.

256. Ranchoux B, Nadeau V, Bourgeois A, Provencher S, Tremblay E, Omura J, Cote N, Abu-Alhayja'a R, Dumais V, Nachbar RT, Tastet L, Dahou A, Breuils-Bonnet S, Marette A, Pibarot P, Dupuis J, Boucherat O, Paulin R, Archer SL, Bonnet S, and Potus F. Metabolic Syndrome Exacerbates Pulmonary Hypertension due to Left Heart Disease. Circ Res 2019.

257. Reeve HL, Michelakis E, Nelson DP, Weir EK, and Archer SL. Alterations in a redox oxygen sensing mechanism in chronic hypoxia. J Appl Physiol (1985) 90: 2249-2256, 2001.

258. Rehman J, and Archer SL. A proposed mitochondrial-metabolic mechanism for initiation and maintenance of pulmonary arterial hypertension in fawn-hooded rats: the Warburg model of pulmonary arterial hypertension. Adv Exp Med Biol 661: 171-185, 2010. 259. Rehman J, Zhang HJ, Toth PT, Zhang Y, Marsboom G, Hong Z, Salgia R, Husain AN, Wietholt C, and Archer SL. Inhibition of mitochondrial fission prevents cell cycle progression in lung cancer. FASEB journal : official publication of the Federation of American Societies for Experimental Biology 26: 2175-2186, 2012.

260. Rey-Parra GJ, Archer SL, Bland RD, Albertine KH, Carlton DP, Cho SC, Kirby B, Haromy A, Eaton F, Wu X, and Thebaud B. Blunted hypoxic pulmonary vasoconstriction in experimental neonatal chronic lung disease. American journal of respiratory and critical care medicine 178: 399-406, 2008.

261. Rhodes CJ, Howard LS, Busbridge M, Ashby D, Kondili E, Gibbs JS, Wharton J, and Wilkins MR. Iron deficiency and raised hepcidin in idiopathic pulmonary arterial hypertension: clinical prevalence, outcomes, and mechanistic insights. J Am Coll Cardiol 58: 300-309, 2011.

262. Rhodes CJ, Wharton J, Boon RA, Roexe T, Tsang H, Wojciak-Stothard B, Chakrabarti A, Howard LS, Gibbs JS, Lawrie A, Condliffe R, Elliot CA, Kiely DG, Huson L, Ghofrani HA, Tiede H, Schermuly R, Zeiher AM, Dimmeler S, and Wilkins MR. Reduced microRNA-150 is associated with poor survival in pulmonary arterial hypertension. American journal of respiratory and critical care medicine 187: 294-302, 2013.

263. Rieusset J, Auwerx J, and Vidal H. Regulation of gene expression by activation of the peroxisome proliferator-activated receptor gamma with rosiglitazone (BRL 49653) in human adipocytes. Biochem Biophys Res Commun 265: 265-271, 1999.

264. Rocchetti M, Sala L, Rizzetto R, Staszewsky LI, Alemanni M, Zambelli V, Russo I, Barile L, Cornaghi L, Altomare C, Ronchi C, Mostacciuolo G, Lucchetti J, Gobbi M, Latini R, and Zaza A. Ranolazine prevents INaL enhancement and blunts myocardial remodelling in a model of pulmonary hypertension. Cardiovasc Res 104: 37-48, 2014. 265. Roh J, Houstis N, and Rosenzweig A. Why Don't We Have Proven Treatments for 
HFpEF? Circ Res 120: 1243-1245, 2017.

266. Rothman AM, Arnold ND, Pickworth JA, Iremonger J, Ciuclan L, Allen RM, GuthGundel S, Southwood M, Morrell NW, Thomas M, Francis SE, Rowlands DJ, and Lawrie A. MicroRNA-140-5p and SMURF1 regulate pulmonary arterial hypertension. J Clin Invest 126: 2495-2508, 2016.

267. Ruiter G, Lankhorst S, Boonstra A, Postmus PE, Zweegman S, Westerhof N, van der Laarse WJ, and Vonk-Noordegraaf A. Iron deficiency is common in idiopathic pulmonary arterial hypertension. Eur Respir J 37: 1386-1391, 2011.

268. Ruiter G, Manders E, Happé CM, Schalij I, Groepenhoff H, Howard LS, Wilkins MR, Bogaard HJ, Westerhof N, van der Laarse WJ, de Man FS, and Vonk-Noordegraaf A. Intravenous iron therapy in patients with idiopathic pulmonary arterial hypertension and iron deficiency. Pulm Circ 5: 466-472, 2015.

269. Ruiter G, Ying Wong Y, de Man FS, Louis Handoko M, Jaspers RT, Postmus PE, Westerhof N, Niessen HW, van der Laarse WJ, and Vonk-Noordegraaf A. Right ventricular oxygen supply parameters are decreased in human and experimental pulmonary hypertension. J Heart Lung Transplant 32: 231-240, 2013.

270. Ryan J, Dasgupta A, Huston J, Chen KH, and Archer SL. Mitochondrial dynamics in pulmonary arterial hypertension. J Mol Med (Berl) 93: 229-242, 2015.

271. Ryan JJ, and Archer SL. Emerging concepts in the molecular basis of pulmonary arterial hypertension: part I: metabolic plasticity and mitochondrial dynamics in the pulmonary circulation and right ventricle in pulmonary arterial hypertension. Circulation 131: 1691-1702, 2015.

272. Ryan JJ, Huston J, Kutty S, Hatton ND, Bowman L, Tian L, Herr JE, Johri AM, and Archer SL. Right ventricular adaptation and failure in pulmonary arterial hypertension. Can J Cardiol 31: 391-406, 2015.

273. Ryan JJ, Marsboom G, Fang YH, Toth PT, Morrow E, Luo N, Piao L, Hong Z, Ericson K, Zhang HJ, Han M, Haney CR, Chen CT, Sharp WW, and Archer SL. PGC1alphamediated mitofusin-2 deficiency in female rats and humans with pulmonary arterial hypertension. American journal of respiratory and critical care medicine 187: 865-878, 2013.

274. Sagan L. On the origin of mitosing cells. J Theor Biol 14: 255-274, 1967.

275. Sahoo N, Hoshi T, and Heinemann SH. Oxidative modulation of voltage-gated potassium channels. Antioxid Redox Signal 21: 933-952, 2014.

276. Samant SA, Zhang HJ, Hong Z, Pillai VB, Sundaresan NR, Wolfgeher D, Archer SL, Chan DC, and Gupta MP. SIRT3 deacetylates and activates OPA1 to regulate mitochondrial dynamics during stress. Mol Cell Biol 34: 807-819, 2014.

277. Saygin D, Highland KB, Farha S, Park M, Sharp J, Roach EC, Tang WHW, Thomas JD, Erzurum SC, Neumann DR, and DiFilippo FP. Metabolic and Functional Evaluation of the Heart and Lungs in Pulmonary Hypertension by Gated 2-[18F]-Fluoro-2-deoxy-Dglucose Positron Emission Tomography. Pulm Circ 7: 428-438, 2017.

278. Schiattarella GG, Altamirano F, Tong D, French KM, Villalobos E, Kim SY, Luo X, Jiang N, May HI, Wang ZV, Hill TM, Mammen PPA, Huang J, Lee DI, Hahn VS, Sharma K, Kass DA, Lavandero S, Gillette TG, and Hill JA. Nitrosative stress drives heart failure with preserved ejection fraction. Nature 568: 351-356, 2019.

279. Schlosser K, White RJ, and Stewart DJ. miR-26a linked to pulmonary hypertension by global assessment of circulating extracellular microRNAs. American journal of 
respiratory and critical care medicine 188: 1472-1475, 2013.

280. Scott I, and Youle RJ. Mitochondrial fission and fusion. Essays Biochem 47: 85-98, 2010.

281. Serasinghe MN, Wieder SY, Renault TT, Elkholi R, Asciolla JJ, Yao JL, Jabado O, Hoehn K, Kageyama Y, Sesaki H, and Chipuk JE. Mitochondrial division is requisite to RAS-induced transformation and targeted by oncogenic MAPK pathway inhibitors. Mol Cell 57: 521-536, 2015.

282. Shah SJ, Kitzman DW, Borlaug BA, van Heerebeek L, Zile MR, Kass DA, and Paulus WJ. Phenotype-Specific Treatment of Heart Failure With Preserved Ejection Fraction: A Multiorgan Roadmap. Circulation 134: 73-90, 2016.

283. Sharp WW, and Archer SL. Mitochondrial dynamics in cardiovascular disease: fission and fusion foretell form and function. J Mol Med (Berl) 93: 225-228, 2015.

284. Shi ZL, Fang K, Li ZH, Ren DH, Zhang JY, and Sun J. EZH2 Inhibition Ameliorates Transverse Aortic Constriction-Induced Pulmonary Arterial Hypertension in Mice. Can Respir J 2018: 9174926, 2018.

285. Shimoda LA, Wang J, and Sylvester JT. Ca2+ channels and chronic hypoxia. Microcirculation 13: 657-670, 2006.

286. Shirai M, Ninomiya I, and Sada K. Constrictor response of small pulmonary arteries to acute pulmonary hypertension during left atrial pressure elevation. The Japanese journal of physiology 41: 129-142, 1991.

287. Silva-Costa-Gomes T, Gallart L, Valles J, Trillo L, Minguella J, and Puig MM. Lowvs high-dose almitrine combined with nitric oxide to prevent hypoxia during open-chest one-lung ventilation. British journal of anaesthesia 95: 410-416, 2005.

288. Simonneau G, Montani D, Celermajer DS, Denton CP, Gatzoulis MA, Krowka M, Williams PG, and Souza R. Haemodynamic definitions and updated clinical classification of pulmonary hypertension. Eur Respir J 53: 2019.

289. Singh N, Manhas A, Kaur G, Jagavelu K, and Hanif K. Inhibition of fatty acid synthase is protective in pulmonary hypertension. BrJ Pharmacol 173: 2030-2045, 2016. 290. Singh N, Singh H, Jagavelu K, Wahajuddin M, and Hanif K. Fatty acid synthase modulates proliferation, metabolic functions and angiogenesis in hypoxic pulmonary artery endothelial cells. Eur J Pharmacol 815: 462-469, 2017.

291. Smith ZD, and Meissner A. DNA methylation: roles in mammalian development. Nat Rev Genet 14: 204-220, 2013.

292. Sommer N, Huttemann M, Pak O, Scheibe S, Knoepp F, Sinkler C, Malczyk M, Gierhardt M, Esfandiary A, Kraut S, Jonas F, Veith C, Aras S, Sydykov A, Alebrahimdehkordi N, Giehl K, Hecker M, Brandes RP, Seeger W, Grimminger F, Ghofrani HA, Schermuly RT, Grossman LI, and Weissmann N. Mitochondrial Complex IV Subunit 4 Isoform 2 Is Essential for Acute Pulmonary Oxygen Sensing. Circulation research 121: 424-438, 2017.

293. Song Z, Chen H, Fiket M, Alexander C, and Chan DC. OPA1 processing controls mitochondrial fusion and is regulated by mRNA splicing, membrane potential, and Yme1L.J Cell Biol 178: 749-755, 2007.

294. Song Z, Ghochani M, McCaffery JM, Frey TG, and Chan DC. Mitofusins and OPA1 mediate sequential steps in mitochondrial membrane fusion. Mol Biol Cell 20: 3525-3532, 2009.

295. Stacpoole PW. The pharmacology of dichloroacetate. Metabolism: clinical and 
experimental 38: 1124-1144, 1989.

296. Stanley WC, Lopaschuk GD, Hall JL, and McCormack JG. Regulation of myocardial carbohydrate metabolism under normal and ischaemic conditions. Potential for pharmacological interventions. Cardiovasc Res 33: 243-257, 1997.

297. Stearman RS, Bui QM, Speyer G, Handen A, Cornelius AR, Graham BB, Kim S, Mickler EA, Tuder RM, Chan SY, and Geraci MW. Systems Analysis of the Human Pulmonary Arterial Hypertension Lung Transcriptome. Am J Respir Cell Mol Biol 60: 637649, 2019.

298. Stojanovski D, Koutsopoulos OS, Okamoto K, and Ryan MT. Levels of human Fis1 at the mitochondrial outer membrane regulate mitochondrial morphology. J Cell Sci 117: 1201-1210, 2004.

299. Sugiura A, Nagashima S, Tokuyama T, Amo T, Matsuki Y, Ishido S, Kudo Y, McBride HM, Fukuda T, Matsushita N, Inatome R, and Yanagi S. MITOL regulates endoplasmic reticulum-mitochondria contacts via Mitofusin2. Molecular cell 51: 20-34, 2013.

300. Sun X, Kumar S, Sharma S, Aggarwal S, Lu Q, Gross C, Rafikova O, Lee SG, Dasarathy S, Hou Y, Meadows ML, Han W, Su Y, Fineman JR, and Black SM. Endothelin1 induces a glycolytic switch in pulmonary arterial endothelial cells via the mitochondrial translocation of endothelial nitric oxide synthase. Am J Respir Cell Mol Biol 50: 1084-1095, 2014

301. Sutendra G, Bonnet S, Rochefort G, Haromy A, Folmes KD, Lopaschuk GD, Dyck JR, and Michelakis ED. Fatty acid oxidation and malonyl-CoA decarboxylase in the vascular remodeling of pulmonary hypertension. Sci Transl Med 2: 44ra58, 2010.

302. Sutendra G, Dromparis P, Paulin R, Zervopoulos S, Haromy A, Nagendran J, and Michelakis ED. A metabolic remodeling in right ventricular hypertrophy is associated with decreased angiogenesis and a transition from a compensated to a decompensated state in pulmonary hypertension. J Mol Med (Berl) 91: 1315-1327, 2013.

303. Sutendra G, Dromparis P, Wright P, Bonnet S, Haromy A, Hao Z, McMurtry MS, Michalak M, Vance JE, Sessa WC, and Michelakis ED. The role of Nogo and the mitochondria-endoplasmic reticulum unit in pulmonary hypertension. Sci Transl Med 3: 88ra55, 2011.

304. Sutendra G, and Michelakis ED. The metabolic basis of pulmonary arterial hypertension. Cell Metab 19: 558-573, 2014.

305. Svoboda LK, Reddie KG, Zhang L, Vesely ED, Williams ES, Schumacher SM, O'Connell RP, Shaw R, Day SM, Anumonwo JM, Carroll KS, and Martens JR. Redoxsensitive sulfenic acid modification regulates surface expression of the cardiovascular voltage-gated potassium channel Kv1.5. Circ Res 111: 842-853, 2012.

306. Taki J, Nakajima K, Matsunari I, Bunko H, Takata S, Kawasuji M, and Tonami N. Assessment of improvement of myocardial fatty acid uptake and function after revascularization using iodine-123-BMIPP. J Nucl Med 38: 1503-1510, 1997.

307. Teng RJ, Jing X, Michalkiewicz T, Afolayan AJ, Wu TJ, and Konduri GG.

Attenuation of endoplasmic reticulum stress by caffeine ameliorates hyperoxia-induced lung injury. American journal of physiology Lung cellular and molecular physiology 312: L586-L598, 2017.

308. Teshima Y, Akao M, Jones SP, and Marban E. Uncoupling protein-2 overexpression inhibits mitochondrial death pathway in cardiomyocytes. Circ Res 93: 192- 
200, 2003.

309. Thebaud B, Wu XC, Kajimoto H, Bonnet S, Hashimoto K, Michelakis ED, and Archer SL. Developmental absence of the 02 sensitivity of L-type calcium channels in preterm ductus arteriosus smooth muscle cells impairs 02 constriction contributing to patent ductus arteriosus. Pediatric research 63: 176-181, 2008.

310. Tian L, Neuber-Hess M, Mewburn J, Dasgupta A, Dunham-Snary K, Wu D, Chen KH, Hong Z, Sharp WW, Kutty S, and Archer SL. Ischemia-induced Drp1 and Fis1mediated mitochondrial fission and right ventricular dysfunction in pulmonary hypertension.J Mol Med (Berl) 95: 381-393, 2017.

311. Tian L, Potus F, Wu D, Dasgupta A, Chen KH, Mewburn J, Lima P, and Archer SL. Increased Drp1-Mediated Mitochondrial Fission Promotes Proliferation and Collagen Production by Right Ventricular Fibroblasts in Experimental Pulmonary Arterial Hypertension. Front Physiol 9: 828, 2018.

312. Tilokani L, Nagashima S, Paupe V, and Prudent J. Mitochondrial dynamics: overview of molecular mechanisms. Essays Biochem 62: 341-360, 2018.

313. Toyama EQ, Herzig S, Courchet J, Lewis TL, Jr., Loson OC, Hellberg K, Young NP, Chen H, Polleux F, Chan DC, and Shaw RJ. Metabolism. AMP-activated protein kinase mediates mitochondrial fission in response to energy stress. Science 351: 275-281, 2016. 314. Trimmer C, Sotgia F, Whitaker-Menezes D, Balliet RM, Eaton G, MartinezOutschoorn UE, Pavlides S, Howell A, Iozzo RV, Pestell RG, Scherer PE, Capozza F, and Lisanti MP. Caveolin-1 and mitochondrial SOD2 (MnSOD) function as tumor suppressors in the stromal microenvironment: a new genetically tractable model for human cancer associated fibroblasts. Cancer Biol Ther 11: 383-394, 2011.

315. Tyagi S, Gupta P, Saini AS, Kaushal C, and Sharma S. The peroxisome proliferatoractivated receptor: A family of nuclear receptors role in various diseases. J Adv Pharm Technol Res 2: 236-240, 2011.

316. Urboniene D, Haber I, Fang YH, Thenappan T, and Archer SL. Validation of highresolution echocardiography and magnetic resonance imaging vs. high-fidelity catheterization in experimental pulmonary hypertension. Am J Physiol Lung Cell Mol Physiol 299: L401-412, 2010.

317. van de Veerdonk MC, Marcus JT, Bogaard HJ, and Vonk Noordegraaf A. State of the art: advanced imaging of the right ventricle and pulmonary circulation in humans (2013 Grover Conference series). Pulm Circ 4: 158-168, 2014.

318. Vega RB, Horton JL, and Kelly DP. Maintaining ancient organelles: mitochondrial biogenesis and maturation. Circ Res 116: 1820-1834, 2015.

319. Veith C, Schermuly RT, Brandes RP, and Weissmann N. Molecular mechanisms of hypoxia-inducible factor-induced pulmonary arterial smooth muscle cell alterations in pulmonary hypertension. The Journal of physiology 594: 1167-1177, 2016.

320. Vogler S, Goedde R, Miterski B, Gold R, Kroner A, Koczan D, Zettl UK, Rieckmann P, Epplen JT, and Ibrahim SM. Association of a common polymorphism in the promoter of UCP2 with susceptibility to multiple sclerosis. J Mol Med (Berl) 83: 806-811, 2005.

321. Vultur A, Gibhardt CS, Stanisz H, and Bogeski I. The role of the mitochondrial calcium uniporter (MCU) complex in cancer. Pflugers Arch 2018.

322. Wang GL, Jiang BH, Rue EA, and Semenza GL. Hypoxia-inducible factor 1 is a basic-helix-loop-helix-PAS heterodimer regulated by cellular 02 tension. Proc Natl Acad Sci 
U S A 92: 5510-5514, 1995.

323. Wang YX, and Zheng YM. ROS-dependent signaling mechanisms for hypoxic $\mathrm{Ca}(2+)$ responses in pulmonary artery myocytes. Antioxid Redox Signal 12: 611-623, 2010.

324. Warburg 0. On the origin of cancer cells. Science 123: 309-314, 1956.

325. Ward JP. Point: Hypoxic pulmonary vasoconstriction is mediated by increased production of reactive oxygen species. J Appl Physiol (1985) 101: 993-995; discussion 999, 2006.

326. Ward JP, and McMurtry IF. Mechanisms of hypoxic pulmonary vasoconstriction and their roles in pulmonary hypertension: new findings for an old problem. Current opinion in pharmacology 9: 287-296, 2009.

327. Waypa GB, Chandel NS, and Schumacker PT. Model for hypoxic pulmonary vasoconstriction involving mitochondrial oxygen sensing. Circ Res 88: 1259-1266, 2001. 328. Waypa GB, Marks JD, Guzy RD, Mungai PT, Schriewer JM, Dokic D, Ball MK, and Schumacker PT. Superoxide generated at mitochondrial complex III triggers acute responses to hypoxia in the pulmonary circulation. American journal of respiratory and critical care medicine 187: 424-432, 2013.

329. Waypa GB, Marks JD, Mack MM, Boriboun C, Mungai PT, and Schumacker PT. Mitochondrial reactive oxygen species trigger calcium increases during hypoxia in pulmonary arterial myocytes. Circ Res 91: 719-726, 2002.

330. Waypa GB, and Schumacker PT. Hypoxic pulmonary vasoconstriction: redox events in oxygen sensing. J Appl Physiol (1985) 98: 404-414, 2005.

331. Weir EK, and Archer SL. Counterpoint: Hypoxic pulmonary vasoconstriction is not mediated by increased production of reactive oxygen species. J Appl Physiol (1985) 101: 995-998; discussion 998, 2006.

332. Weir EK, and Archer SL. The mechanism of acute hypoxic pulmonary vasoconstriction: the tale of two channels. FASEB journal : official publication of the Federation of American Societies for Experimental Biology 9: 183-189, 1995.

333. Weir EK, Lopez-Barneo J, Buckler KJ, and Archer SL. Acute oxygen-sensing mechanisms. N Engl J Med 353: 2042-2055, 2005.

334. Weiss G, and Goodnough LT. Anemia of chronic disease. N Engl J Med 352: 10111023, 2005.

335. Weissmann N, Dietrich A, Fuchs B, Kalwa H, Ay M, Dumitrascu R, Olschewski A, Storch U, Mederos y Schnitzler M, Ghofrani HA, Schermuly RT, Pinkenburg 0, Seeger W, Grimminger F, and Gudermann T. Classical transient receptor potential channel 6 (TRPC6) is essential for hypoxic pulmonary vasoconstriction and alveolar gas exchange. Proc Natl Acad Sci U S A 103: 19093-19098, 2006.

336. White K, Lu Y, Annis S, Hale AE, Chau BN, Dahlman JE, Hemann C, Opotowsky AR, Vargas SO, Rosas I, Perrella MA, Osorio JC, Haley KJ, Graham BB, Kumar R, Saggar R, Saggar R, Wallace WD, Ross DJ, Khan OF, Bader A, Gochuico BR, Matar M, Polach K, Johannessen NM, Prosser HM, Anderson DG, Langer R, Zweier JL, Bindoff LA, Systrom D, Waxman AB, Jin RC, and Chan SY. Genetic and hypoxic alterations of the microRNA210-ISCU1/2 axis promote iron-sulfur deficiency and pulmonary hypertension. EMBO Mol Med 7: 695-713, 2015.

337. Whitley BN, Lam C, Cui H, Haude K, Bai R, Escobar L, Hamilton A, Brady L, Tarnopolsky MA, Dengle L, Picker J, Lincoln S, Lackner LL, Glass IA, and Hoppins S. Aberrant Drp1-mediated mitochondrial division presents in humans with variable 
outcomes. Hum Mol Genet 27: 3710-3719, 2018.

338. Wijeratne DT, Lajkosz K, Brogly SB, Lougheed MD, Jiang L, Housin A, Barber D, Johnson A, Doliszny KM, and Archer SL. Increasing Incidence and Prevalence of World Health Organization Groups 1 to 4 Pulmonary Hypertension: A Population-Based Cohort Study in Ontario, Canada. Circ Cardiovasc Qual Outcomes 11: e003973, 2018.

339. Wolff AA, Rotmensch HH, Stanley WC, and Ferrari R. Metabolic approaches to the treatment of ischemic heart disease: the clinicians' perspective. Heart Fail Rev 7: 187-203, 2002.

340. Wolffe AP, and Matzke MA. Epigenetics: regulation through repression. Science 286: 481-486, 1999.

341. Xiong PY, Tian L, Dunham-Snary KJ, Chen KH, Mewburn JD, Neuber-Hess M, Martin A, Dasgupta A, Potus F, and Archer SL. Biventricular Increases in Mitochondrial Fission Mediator (MiD51) and Proglycolytic Pyruvate Kinase (PKM2) Isoform in

Experimental Group 2 Pulmonary Hypertension-Novel Mitochondrial Abnormalities. Front Cardiovasc Med 5: 195, 2018.

342. Xu K, Chen G, Li X, Wu X, Chang Z, Xu J, Zhu Y, Yin P, Liang X, and Dong L. MFN2 suppresses cancer progression through inhibition of mTORC2/Akt signaling. Sci Rep 7: 41718, 2017.

343. Xu S, Cherok E, Das S, Li S, Roelofs BA, Ge SX, Polster BM, Boyman L, Lederer WJ, Wang C, and Karbowski M. Mitochondrial E3 ubiquitin ligase MARCH5 controls mitochondrial fission and cell sensitivity to stress-induced apoptosis through regulation of MiD49 protein. Mol Biol Cell 27: 349-359, 2016.

344. Xu S, Wang P, Zhang H, Gong G, Gutierrez Cortes N, Zhu W, Yoon Y, Tian R, and Wang W. CaMKII induces permeability transition through Drp1 phosphorylation during chronic beta-AR stimulation. Nat Commun 7: 13189, 2016.

345. Xu W, Koeck T, Lara AR, Neumann D, DiFilippo FP, Koo M, Janocha AJ, Masri FA, Arroliga AC, Jennings C, Dweik RA, Tuder RM, Stuehr DJ, and Erzurum SC. Alterations of cellular bioenergetics in pulmonary artery endothelial cells. Proc Natl Acad Sci U S A 104: 1342-1347, 2007.

346. Yang J, Zhang L, Erbel PJ, Gardner KH, Ding K, Garcia JA, and Bruick RK.

Functions of the Per/ARNT/Sim domains of the hypoxia-inducible factor.J Biol Chem 280: 36047-36054, 2005.

347. Yang R, Lirussi D, Thornton TM, Jelley-Gibbs DM, Diehl SA, Case LK, Madesh M, Taatjes DJ, Teuscher C, Haynes L, and Rincon M. Mitochondrial $\mathrm{Ca}(2)(+)$ and membrane potential, an alternative pathway for Interleukin 6 to regulate CD4 cell effector function. Elife 4: 2015.

348. Ye JX, Wang SS, Ge M, and Wang DJ. Suppression of endothelial PGC-1alpha is associated with hypoxia-induced endothelial dysfunction and provides a new therapeutic target in pulmonary arterial hypertension. American journal of physiology Lung cellular and molecular physiology 310: L1233-1242, 2016.

349. Yeaman SJ, Hutcheson ET, Roche TE, Pettit FH, Brown JR, Reed LJ, Watson DC, and Dixon GH. Sites of phosphorylation on pyruvate dehydrogenase from bovine kidney and heart. Biochemistry 17: 2364-2370, 1978.

350. Yeligar SM, Kang BY, Bijli KM, Kleinhenz JM, Murphy TC, Torres G, San Martin A, Sutliff RL, and Hart CM. PPARgamma Regulates Mitochondrial Structure and Function and Human Pulmonary Artery Smooth Muscle Cell Proliferation. Am J Respir Cell Mol Biol 
58: 648-657, 2018.

351. Yoon Y, Krueger EW, Oswald BJ, and McNiven MA. The mitochondrial protein hFis1 regulates mitochondrial fission in mammalian cells through an interaction with the dynamin-like protein DLP1. Mol Cell Biol 23: 5409-5420, 2003.

352. Youle RJ, and Narendra DP. Mechanisms of mitophagy. Nat Rev Mol Cell Biol 12: 9$14,2011$.

353. Yu AY, Frid MG, Shimoda LA, Wiener CM, Stenmark K, and Semenza GL. Temporal, spatial, and oxygen-regulated expression of hypoxia-inducible factor- 1 in the lung. Am J Physiol 275: L818-826, 1998.

354. Yu F, White SB, Zhao Q, and Lee FS. HIF-1alpha binding to VHL is regulated by stimulus-sensitive proline hydroxylation. Proc Natl Acad Sci U S A 98: 9630-9635, 2001. 355. Yu R, Jin SB, Lendahl U, Nister M, and Zhao J. Human Fis1 regulates mitochondrial dynamics through inhibition of the fusion machinery. EMBO J 38: 2019.

356. Yuan K, Shao NY, Hennigs JK, Discipulo M, Orcholski ME, Shamskhou E, Richter A, Hu X, Wu JC, and de Jesus Perez VA. Increased Pyruvate Dehydrogenase Kinase 4 Expression in Lung Pericytes Is Associated with Reduced Endothelial-Pericyte Interactions and Small Vessel Loss in Pulmonary Arterial Hypertension. Am J Pathol 186: 2500-2514, 2016.

357. Yuan XJ, Tod ML, Rubin LJ, and Blaustein MP. Contrasting effects of hypoxia on tension in rat pulmonary and mesenteric arteries. Am J Physiol 259: H281-289, 1990. 358. Zamponi GW, Striessnig J, Koschak A, and Dolphin AC. The Physiology, Pathology, and Pharmacology of Voltage-Gated Calcium Channels and Their Future Therapeutic Potential. Pharmacol Rev 67: 821-870, 2015.

359. Zhang C, Ma C, Zhang L, Zhang L, Zhang F, Ma M, Zheng X, Mao M, Shen T, and Zhu D. MiR-449a-5p mediates mitochondrial dysfunction and phenotypic transition by targeting Myc in pulmonary arterial smooth muscle cells. J Mol Med (Berl) 97: 409-422, 2019.

360. Zhang H, Wang D, Li M, Plecita-Hlavata L, D'Alessandro A, Tauber J, Riddle S, Kumar S, Flockton A, McKeon BA, Frid MG, Reisz JA, Caruso P, El Kasmi KC, Jezek P, Morrell NW, Hu CJ, and Stenmark KR. Metabolic and Proliferative State of Vascular Adventitial Fibroblasts in Pulmonary Hypertension Is Regulated Through a MicroRNA124/PTBP1 (Polypyrimidine Tract Binding Protein 1)/Pyruvate Kinase Muscle Axis. Circulation 136: 2468-2485, 2017.

361. Zhao L, Ashek A, Wang L, Fang W, Dabral S, Dubois O, Cupitt J, Pullamsetti SS, Cotroneo E, Jones H, Tomasi G, Nguyen QD, Aboagye EO, El-Bahrawy MA, Barnes G, Howard LS, Gibbs JS, Gsell W, He JG, and Wilkins MR. Heterogeneity in lung (18)FDG uptake in pulmonary arterial hypertension: potential of dynamic (18)FDG positron emission tomography with kinetic analysis as a bridging biomarker for pulmonary vascular remodeling targeted treatments. Circulation 128: 1214-1224, 2013.

362. Zhao L, Chen CN, Hajji N, Oliver E, Cotroneo E, Wharton J, Wang D, Li M, McKinsey TA, Stenmark KR, and Wilkins MR. Histone deacetylation inhibition in pulmonary hypertension: therapeutic potential of valproic acid and suberoylanilide hydroxamic acid. Circulation 126: 455-467, 2012.

363. Zuchner S, Mersiyanova IV, Muglia M, Bissar-Tadmouri N, Rochelle J, Dadali EL, Zappia M, Nelis E, Patitucci A, Senderek J, Parman Y, Evgrafov O, Jonghe PD, Takahashi Y, Tsuji S, Pericak-Vance MA, Quattrone A, Battaloglu E, Polyakov AV, 
Timmerman V, Schroder JM, and Vance JM. Mutations in the mitochondrial GTPase mitofusin 2 cause Charcot-Marie-Tooth neuropathy type 2A. Nat Genet 36: 449-451, 2004. 364. Zurlo G, Piquereau J, Moulin M, Pires Da Silva J, Gressette M, Ranchoux B, Garnier A, Ventura-Clapier R, Fadel E, Humbert M, Lemaire C, Perros F, and Veksler V. Sirtuin 1 regulates pulmonary artery smooth muscle cell proliferation: role in pulmonary arterial hypertension. J Hypertens 36: 1164-1177, 2018. 
Table 2. Summary of mitochondrial metabolism, upstream regulators, downstream effectors and associated mitochondrial ROS production, membrane potential and morphology (fission) in (A) endothelial cells, (B) smooth muscle cells, and (C) fibroblasts in pulmonary artery in PAH.

Table 2 (A)

\begin{tabular}{|c|c|c|c|c|c|c|c|}
\hline $\begin{array}{l}\text { Cell type \& } \\
\text { metabolism }\end{array}$ & $\begin{array}{l}\text { Animal } \\
\text { model } / \text { patients }\end{array}$ & $\begin{array}{l}\text { Upstream } \\
\text { regulators }\end{array}$ & $\begin{array}{l}\text { Downstream } \\
\text { effectors }\end{array}$ & mROS & $\Delta \psi_{m}$ & $\begin{array}{l}\text { Mito. } \\
\text { fission }\end{array}$ & Ref. \\
\hline \multirow[t]{6}{*}{$\begin{array}{l}\text { EC: } \\
\text { Glycolysis }\end{array}$} & IPAH & $\begin{array}{l}\downarrow \text { Complex IV, } \\
\downarrow \text { SOD } 2, \uparrow H I F- \\
1 \alpha, \downarrow N O\end{array}$ & & & & & $(345),(98)$ \\
\hline & $\begin{array}{l}\text { Healthy ovine } \\
\text { PAEC } \\
\text { exposed to } \\
\text { ET-1, MCT } \\
\text { rats }\end{array}$ & $\begin{array}{l}\uparrow \mathrm{ET}-1, \\
\uparrow \mathrm{PKC} \delta, \\
\uparrow \mathrm{eNOS}, \uparrow \mathrm{HIF}- \\
1 \alpha\end{array}$ & & $\uparrow$ & $\downarrow$ & & $(300)$ \\
\hline & $\begin{array}{l}\text { BMPR2 } \\
\text { mutant mice } \\
\text { and PAH } \\
\text { patients in } \\
\text { normoxia }\end{array}$ & $\begin{array}{l}\downarrow \mathrm{BMPR} 2, \\
\uparrow \mathrm{p} 53, \uparrow \mathrm{PGC}- \\
1 \alpha\end{array}$ & & $\begin{array}{l}\uparrow \\
\text { (only } \\
\text { in } \\
\text { mice) }\end{array}$ & $\uparrow$ & $\uparrow$ & (83) \\
\hline & $\begin{array}{l}\text { BMPR2 } \\
\text { mutant mice } \\
\text { and PAH } \\
\text { patients in } \\
\text { Hypoxia }\end{array}$ & $\begin{array}{l}\downarrow \mathrm{BMPR} 2, \\
\downarrow \mathrm{p} 53, \downarrow \text { PGC- } \\
1 \alpha\end{array}$ & & $\begin{array}{l}\downarrow \\
\text { (only } \\
\text { in } \\
\text { mice) }\end{array}$ & $\downarrow$ & $\uparrow$ & (83) \\
\hline & IPAH, HPAH & $\begin{array}{l}\downarrow \mathrm{miR} 124, \\
\uparrow \mathrm{PTBP} 1, \\
\uparrow \mathrm{PKM} 2, \\
\uparrow \mathrm{MCT} 1, \\
\uparrow \mathrm{LDHA}\end{array}$ & & & & & (45) \\
\hline & $\begin{array}{l}\text { IPAH, } \\
\text { hypoxic mice, } \\
\text { Sugen- } \\
\text { Hypoxia rats }\end{array}$ & $\begin{array}{l}\uparrow P F K F B 3, \uparrow F- \\
2,6-P 2\end{array}$ & $\begin{array}{l}\uparrow P D G F B, \\
\uparrow F G F 2, \\
\uparrow C X C L 12, \\
\uparrow I L 1 \beta\end{array}$ & & & & (44) \\
\hline $\begin{array}{l}\text { EC: } \\
\text { FAO }\end{array}$ & $\begin{array}{l}\text { Healthy } \\
\text { human PAEC } \\
\text { under hypoxia }\end{array}$ & $\begin{array}{l}\uparrow F A S, \uparrow H I F- \\
1 \alpha, \uparrow G l u t 1, \\
\uparrow H K 2, \downarrow P D H \\
\text { activity }\end{array}$ & $\begin{array}{l}\downarrow \mathrm{Bax}, \\
\uparrow \mathrm{Bcl} 2, \downarrow \mathrm{p}- \\
\mathrm{mTOR}, \\
\downarrow \mathrm{p} 62,\end{array}$ & & & & (290) \\
\hline
\end{tabular}




\begin{tabular}{|l|l|l|l|l|l|l|c|}
\hline & & & $\uparrow$ VEGF & & & & \\
\hline $\begin{array}{l}\text { EC: } \\
\text { Glutaminolysis }\end{array}$ & $\begin{array}{l}\text { Human PAEC } \\
\text { under stiff } \\
\text { extracellular } \\
\text { matrix }\end{array}$ & $\begin{array}{l}\uparrow \text { YAPS1, } \uparrow \text { TAZ, } \\
\text { LDHA, } \uparrow \text { PC }\end{array}$ & & & & & $(33)$ \\
\cline { 2 - 7 } & $\begin{array}{l}\text { BMPR2 } \\
\text { mutant mice } \\
\text { and PAH } \\
\text { patients }\end{array}$ & $\begin{array}{l}\downarrow \text { BMPR2, } \\
\text { SIRT3, } \\
\uparrow \text { HIF-1 } \alpha\end{array}$ & & & & & $(88)$ \\
\hline
\end{tabular}


Table 2 (B)

\begin{tabular}{|c|c|c|c|c|c|c|c|}
\hline $\begin{array}{l}\text { Cell type \& } \\
\text { metabolism }\end{array}$ & $\begin{array}{l}\text { Animal } \\
\text { model/patients }\end{array}$ & Upstream regulators & $\begin{array}{l}\text { Downstream } \\
\text { effectors }\end{array}$ & mROS & $\Delta \psi_{m}$ & $\begin{array}{l}\text { Mito. } \\
\text { fission }\end{array}$ & Ref. \\
\hline \multirow[t]{7}{*}{$\begin{array}{l}\text { SMC: } \\
\text { Glycolysis }\end{array}$} & $\begin{array}{l}\text { Fawn-hooded } \\
\text { rats, IPAH } \\
\text { patients }\end{array}$ & $\begin{array}{l}\uparrow \mathrm{DNMT} 1, \uparrow \mathrm{DNMT} 3 \mathrm{~b}, \\
\downarrow \text { SOD } 2, \uparrow \mathrm{HIF}-1 \alpha, \\
\downarrow \text { complex I activity }\end{array}$ & $\downarrow \mathrm{Kv} 1.5$ & $\downarrow$ & $\uparrow$ & $\uparrow$ & $\begin{array}{l}(15),( \\
36)\end{array}$ \\
\hline & $\begin{array}{l}\text { MCT rats, } \\
\text { Sugen-Hypoxia } \\
\text { rats }\end{array}$ & $\begin{array}{l}\uparrow \text { Glut } 1, \uparrow \mathrm{HK} 1, \uparrow \mathrm{HIF}- \\
1 \alpha, \uparrow \mathrm{PDK} 1, \uparrow \mathrm{PDK} 3, \\
\downarrow \mathrm{PDH} \text { activity }\end{array}$ & & & & & (193) \\
\hline & $\begin{array}{l}\text { SIRT3 } \\
\text { knockout mice, } \\
\text { IPAH patients }\end{array}$ & $\begin{array}{l}\downarrow \text { PGC-1 } \alpha, \downarrow \text { SIRT3 } \\
, \downarrow \text { PDH } \\
\text { activity, } \uparrow \text { HIF 1a, } \uparrow \text { PDK1 }\end{array}$ & $\begin{array}{l}\downarrow \text { aKG,GRIM- } \\
\text { 19, } \uparrow \text { STAT3, } \\
\uparrow N F A T c 2, \uparrow p- \\
\text { GSK-3b, } \\
\downarrow \text { Kv1.5 }\end{array}$ & & $\uparrow$ & & $(242)$ \\
\hline & MCT rats & $\downarrow$ Complex I activity & & $\uparrow$ & $\uparrow$ & & $(255)$ \\
\hline & $\begin{array}{l}\text { MCT rats, } \\
\text { Sugen-Hypoxia } \\
\text { rats, PAH } \\
\text { patients }\end{array}$ & $\begin{array}{l}\uparrow \mathrm{miR}-25, \uparrow \mathrm{miR}-138, \\
\downarrow \mathrm{MCU}, \downarrow \mathrm{MCU} 1, \\
\downarrow \mathrm{CREB}, \downarrow \mathrm{PDH} \\
\text { activity }\end{array}$ & & & & $\uparrow$ & $(140)$ \\
\hline & $\begin{array}{l}\text { MCT rats, } \\
\text { hypoxia rats }\end{array}$ & $\downarrow \mathrm{miR}-449-5 \mathrm{p}, \uparrow \mathrm{Myc}$ & & $\uparrow$ & & $\uparrow$ & (359) \\
\hline & $\begin{array}{l}\text { Sugen-Hypoxia } \\
\text { rats, PAH } \\
\text { patients }\end{array}$ & $\begin{array}{l}\uparrow P F K F B 3, \uparrow E R K 1 / 2, \\
\uparrow \text { calpain-2 }\end{array}$ & & & & & $(160)$ \\
\hline \multirow[t]{2}{*}{$\begin{array}{l}\text { SMC: } \\
\text { FAO }\end{array}$} & $\begin{array}{l}\text { MCD knockout } \\
\text { mice }\end{array}$ & $\downarrow \mathrm{MCD}$ & $\begin{array}{l}\text { NFAtC2, } \\
\text { Kv1.5, GSK- } \\
3 b\end{array}$ & - & - & & (301) \\
\hline & $\begin{array}{l}\text { Healthy human } \\
\text { PASMC under } \\
\text { hypoxia }\end{array}$ & $\begin{array}{l}\uparrow F A S, \uparrow \text { Glut } 1, \\
\uparrow p G S K 3 b, \downarrow \text { PDH } \\
\text { activity, } \uparrow \text { CPT-1 }\end{array}$ & $\begin{array}{l}\downarrow \text { Bax, } \uparrow \mathrm{Bcl} 2, \\
\downarrow \text { caspase3, } \\
\downarrow \text { annexinV, } \\
\downarrow \text { mTOR, } \\
\downarrow \text { p62, } \\
\uparrow \mathrm{LC} 3 \mathrm{BII} / \mathrm{I}\end{array}$ & $\downarrow$ & $\uparrow$ & & (289) \\
\hline
\end{tabular}


Table 2 (C)

\begin{tabular}{|c|c|c|c|c|c|c|c|}
\hline $\begin{array}{l}\text { Cell type \& } \\
\text { metabolism }\end{array}$ & $\begin{array}{l}\text { Animal } \\
\text { model/patients }\end{array}$ & $\begin{array}{l}\text { Upstream } \\
\text { regulators }\end{array}$ & $\begin{array}{l}\text { Downstream } \\
\text { effectors }\end{array}$ & mROS & $\Delta \psi_{m}$ & $\begin{array}{l}\text { Mito. } \\
\text { fission }\end{array}$ & Ref. \\
\hline \multirow[t]{4}{*}{$\begin{array}{l}\text { Fib: } \\
\text { Glycolysis }\end{array}$} & IPAH patients & $\begin{array}{l}\uparrow \text { Glut1, } \\
\uparrow \mathrm{PDK} 1, \\
\uparrow \mathrm{HK} 1\end{array}$ & & & & & (361) \\
\hline & $\begin{array}{l}\text { Hypoxic } \\
\text { neonatal } \\
\text { calves, IPAH } \\
\text { patients }\end{array}$ & $\begin{array}{l}\downarrow \text { complex I } \\
\text { activity, } \\
\downarrow \text { NDUFS4, } \\
\downarrow \text { PDH } \\
\text { activity }\end{array}$ & & $\uparrow$ & $\uparrow$ & $\uparrow$ & (246) \\
\hline & $\begin{array}{l}\text { Hypoxic } \\
\text { neonatal } \\
\text { calves, IPAH } \\
\text { patients }\end{array}$ & $\begin{array}{l}\uparrow \text { Glut1, } \\
\uparrow \mathrm{HK} 2, \\
\uparrow \mathrm{LDHA}\end{array}$ & $\begin{array}{l}\uparrow \mathrm{NADH}, \\
\uparrow \mathrm{CtBP} 1, \\
\downarrow \mathrm{P} 21, \downarrow \mathrm{P} 15, \\
\downarrow \mathrm{PERP}, \\
\downarrow \mathrm{NOX}, \\
\downarrow \text { HMOX } 1\end{array}$ & & & & (173) \\
\hline & $\begin{array}{l}\text { Hypoxic } \\
\text { neonatal } \\
\text { calves, IPAH } \\
\text { and HPAH } \\
\text { patients }\end{array}$ & $\begin{array}{l}\downarrow \text { miR-124, } \\
\uparrow \text { PTBP1, } \\
\uparrow \text { PKM2, } \\
\downarrow \text { MPC1, } \\
\downarrow \text { complex I } \\
\text { activity, } \\
\downarrow \text { NDUFS4, } \\
\downarrow \text { PDH } \\
\text { activity }\end{array}$ & & $\uparrow$ & $\uparrow$ & & (360) \\
\hline
\end{tabular}

* FAO, fatty acid oxidation; EC, endothelial cells; SMC, smooth muscle cells; Fib, fibroblasts. mROS, mitochondrial reactive oxygen species; $\Delta \psi_{m}$, mitochondrial membrane potential; $\mathrm{PAH}$, pulmonary arterial hypertension; HPAH, heritable PAH; IPAH, idiopathic PAH; SOD2, mitochondrial superoxide dismutase 2; NO, nitric oxide; HIF-1a, hypoxia-inducible factor 1alpha; ET-1, endothelin-1; eNOS, endothelial nitric oxide synthase; $\mathrm{PKC} \delta$, protein kinase $\mathrm{C} \delta$; BMPR2, bone morphogenetic protein receptor type 2; PTBP1, polypyrimidine-tract-binding protein; PKM2, pyruvate kinase muscle isoform 2; MCT1, monocarboxylate transporter 1; LDHA, Lactate dehydrogenase A; FAS, fatty acid synthase; Glut-1, glucose transporter 1; HK1/2, hexokinase I/II; VEGF, vascular endothelial growth factor; YAP, Yes-associated protein 1; GLS1, glutaminase; PC, pyruvate carboxylase; Sirt3, sirtuin-3; DNMT1/3b, DNA Methyltransferase 1/3b; PDK1/3, Pyruvate dehydrogenase kinase isoforms 1/3; MCU, mitochondrial calcium uniporter; MCU1, mitochondrial calcium uptake protein 1; a-KG, a-ketoglutarate; STAT3, signal transducer and activator of transcription 3; GSK-3b, glycogen synthase kinase 3 beta; NFATc2, nuclear factor of activated T-cells, cytoplasmic 2; PFKFB3, 6-phosphofructo-2-kinase/fructose2,6-bisphosphatase 3; ERK1/2, extracellular signal-regulated kinase 1 and 2; MCD, metabolic 
enzyme malonyl-coenzyme A (CoA) decarboxylase; CPT-1, carnitine palmitoyltransferase I; NDUFS4, NADH dehydrogenase [ubiquinone] iron-sulfur protein 4, mitochondrial; F-2,6-P2, fructosie-2, 6-bisphosphate; PDGFB, platelet derived growth factor subunit B; FGF2 (also known as basic FGF), fibroblast growth factor-2; IL1ß; interleukin 1 beta; CXCL12, C-X-C motif chemokine 12 .

Table 3. Dysregulation of microRNAs affecting mitochondrial pathways in $\mathrm{PH}$

\begin{tabular}{|l|l|l|l|}
\hline Micro-RNA & Role in PH & Targets & Reference \\
\hline miR-17 & $\begin{array}{l}\text { Increased in PAH } \\
\text { PASMC. Promotes } \\
\text { proliferation and apoptosis } \\
\text { resistance }\end{array}$ & Mfn2, p21 (CIP1/WAF1) & (46, 184, 252) \\
\hline miR-25 & $\begin{array}{l}\text { Upregulated in PAH } \\
\text { PASMC resulting in } \\
\text { downregulation of } \\
\text { calcium uniporter (MCU). } \\
\text { This results in increase in } \\
\text { cytosolic calcium and } \\
\text { decrease in } \\
\text { intramitochondrial } \\
\text { calcium promoting a } \\
\text { glycolytic shift which } \\
\text { induces mitochondrial } \\
\text { fission and cell } \\
\text { proliferation. }\end{array}$ & MCU & (140) \\
\hline miR-34a-3p & $\begin{array}{l}\text { Downregulated in PAH } \\
\text { PASMC. miR-34a-3p is a } \\
\text { negative regulator of } \\
\text { mitochondrial dynamics } \\
\text { protein of 49 kDa } \\
\text { (MiD49) and } \\
\text { mitochondrial dynamics } \\
\text { protein of 51 kDa } \\
\text { (MiD51). Downregulation } \\
\text { of miR-34a-3p induces } \\
\text { mitochondrial fission and } \\
\text { cell proliferation in PAH } \\
\text { PASMC via MiD49 and } \\
\text { MiD51 }\end{array}$ & & \\
\hline $\begin{array}{l}\text { Increased miR-138 } \\
\text { downregulated MCU in } \\
\text { PAH PAMC. This results } \\
\text { in the decrease of } \\
\text { intramitochondrial }\end{array}$ & & \\
\hline miR-138 & & \\
\hline
\end{tabular}




\begin{tabular}{|c|c|c|c|}
\hline & $\begin{array}{l}\text { calcium promoting a } \\
\text { promoting a glycolytic } \\
\text { shift which induces } \\
\text { mitochondrial fission and } \\
\text { cell proliferation. }\end{array}$ & & \\
\hline$m i R-210$ (hypoxamiR) & $\begin{array}{l}\text { Increased in vascular and } \\
\text { endothelial tissue by } \\
\text { hypoxia induction. } \\
\text { Impacts energy } \\
\text { metabolism by supressing } \\
\text { genes involved in } \\
\text { mitochondrial respiration, } \\
\text { such as subunit D of } \\
\text { succinate dehydrogenase } \\
\text { complex (SDHD) and } \\
\text { iron-sulfur cluster } \\
\text { assembly proteins } \\
\text { (ISCU1/2). }\end{array}$ & SDHD, ISCU1/2 & $(50,251,336)$ \\
\hline
\end{tabular}


Table 4 Functional Gene Ontology analysis of PAH microarray data

\begin{tabular}{|c|c|c|c|c|}
\hline Category & Term & Count & $\begin{array}{l}\text { Fold } \\
\text { Enrichment }\end{array}$ & Benjamini \\
\hline UP_KEYWORDS & Oxidoreductase & 109 & 1.73 & 0.000001 \\
\hline GOTERM_MF_DIRECT & $\begin{array}{l}\text { GO:0009055 electron } \\
\text { carrier activity }\end{array}$ & 31 & 2.91 & 0.000061 \\
\hline GOTERM_CC_DIRECT & GO:0005739 mitochondrion & 209 & 1.34 & 0.000679 \\
\hline UP_KEYWORDS & NADP & 42 & 2.09 & 0.000211 \\
\hline UP_KEYWORDS & NAD & 40 & 2.12 & 0.000252 \\
\hline UP_KEYWORDS & Lipid biosynthesis & 36 & 2.14 & 0.000555 \\
\hline GOTERM_MF_DIRECT & GO:0051287 NAD binding & 15 & 3.33 & 0.036638 \\
\hline UP_SEQ_FEATURE & $\begin{array}{l}\text { nucleotide phosphate- } \\
\text { binding region:NAD }\end{array}$ & 22 & 2.49 & 0.077793 \\
\hline KEGG_PATHWAY & $\begin{array}{l}\text { hsa00020: Citrate cycle (TCA } \\
\text { cycle) }\end{array}$ & 13 & 3.22 & 0.013275 \\
\hline KEGG_PATHWAY & $\begin{array}{l}\text { hsa01100:Metabolic } \\
\text { pathways }\end{array}$ & 200 & 1.22 & 0.035534 \\
\hline KEGG_PATHWAY & $\begin{array}{l}\text { hsa00620:Pyruvate } \\
\text { metabolism }\end{array}$ & 14 & 2.60 & 0.055426 \\
\hline UP_KEYWORDS & Fatty acid biosynthesis & 14 & 2.49 & 0.032715 \\
\hline UP_KEYWORDS & Mitochondrion & 149 & 1.23 & 0.051518 \\
\hline UP_KEYWORDS & Fatty acid metabolism & 24 & 1.79 & 0.061674 \\
\hline UP_KEYWORDS & Mitochondrion & 43 & 1.47 & 0.078962 \\
\hline
\end{tabular}




\begin{tabular}{|l|l|l|l|l|}
\hline & membrane & & & \\
\hline
\end{tabular}




\section{Figure legends}

\section{Figure 1. System for Homeostatic Oxygen-Sensing.}

This diagram indicates specialized tissues sensing local oxygen level. In response to hypoxia, the carotid body, located at the carotid-artery bifurcation, increases action-potential frequency in the carotid-sinus nerve, thus stimulating respiration. The small resistance pulmonary and fetoplacental arteries exhibit hypoxic vasoconstriction, which optimizes oxygen transfer in the lung and placenta. On the contrary, the ductus arteriosus, contracts with increased oxygen tensions, redirecting blood through the newly expanded lungs of the newborn. The neuroepithelial bodies in the lungs and adrenomedullary cells in the fetus also sense oxygen. (Adapted from Copyright (C) 2019 New England Journal of Medicine.

Figure 2: Ventilation/Perfusion matching in vivo (modified from Ref. J Mol Cell Cardiol. 2004 Dec;37(6):1119-36.): A chest x-ray of a patient with post-operative atelectasis of the right lower lobe. Hypoventilation is observed in the ventilation (V) study and localized hypoxic pulmonary vasoconstriction (HPV) elicits a corresponding reduction in perfusion (Q).

Figure 3A. Schematic of RISP as the mitochondrial oxygen-sensor (modified from Ref. Am J Respir Crit Care Med. 2013 Feb 15; 187(4): 424-432): This schematic illustrates a proposed mechanism by which increased ROS production at RISP, a component of ETC Complex III in response to hypoxia stimulate HPV. This group finds that loss of RISP attenuates acute oxygensensing. $\mathrm{IMS}=$ intermembrane space; $\mathrm{KO}=$ knockout; $\mathrm{NAD}+=$ nicotinamide adenine dinucleotide; $\mathrm{NADH}=\mathrm{NAD}+$ reduced; PASMC $=$ pulmonary arterial smooth muscle cells; RISP 
$=$ Rieske iron-sulfur protein; $\mathrm{ROS}=$ reactive oxygen species; $\mathrm{SASMC}=$ systemic arterial smooth muscle cells; YC2.3-FRET = the calcium-sensitive, Förster resonance energy transfer sensor.

Figure 3B-E: NADH dehydrogenase [ubiquinone] iron-sulfur protein 2 (Ndufs2) is the pulmonary vascular oxygen sensor (modified from Ref. Circ Res. 2019 Mar 29. doi: 10.1161/CIRCRESAHA.118.314284): This work shows the evidence that hypoxic inhibition of ETC complex I (and NDUFS2) reduces mitochondrial derived ROS production, triggering HPV. Schematic showing the pulmonary vasculature and PASMC mitochondria during normoxia (left) and hypoxia (right). B) During normoxia, mitochondrial $\mathrm{H}_{2} \mathrm{O}_{2}$ production and elevated NAD/NADH ratio result in an oxidative environment and leads to oxidation of sulfhydryl groups (S-S) on $\mathrm{Kv}$ channels, thereby increasing their open state probability, while the $\mathrm{Ca}_{\mathrm{L}}$ channel remains closed. C) Hypoxia lowers mitochondrial superoxide and hydrogen peroxide levels which, coupled with accumulation of NADH, result in depolarization of the cell, closing Kv channels and thereby increasing the opening of $\mathrm{CaL}$ channels and triggering HPV. D) Intact Ndufs2 is required for optimal Complex I function, maintenance of circulating normoxic $\mathrm{H}_{2} \mathrm{O}_{2}$ levels and sensing of changes in $\mathrm{O}_{2}$. E) Inhibition of Complex I, whether caused by hypoxic, pharmacological or molecular inhibition of Ndufs2 results in a more reduced SMC redox state, inhibiting Kv channel expression and a loss of activation of the $\mathrm{Ca}_{\mathrm{L}}$ channel and vasoconstriction.

Figure 4: Contradictory roles for ROS in oxygen-sensing and HPV (modified from Ref. J Appl Physiol (1985). 2005 Jan;98(1):404-14.): Left: Hypoxic pulmonary vasoconstriction (HPV) is a result of hypoxia-induced decrease in reactive oxygen species (ROS) signaling. Right: HPV is a result of a paradoxical increase in ROS signaling. It is suspected the differences between these models reflect unstated and unrecognized differences in methodology (related to differences in 
$\mathrm{pH}, \mathrm{PO}_{2}$ etc.), tissues studied (cultured cells versus freshly isolated cells and PA rings) and temporal differences in the phase of HPV studied ( $<15$ minutes versus later).

cADPR - cyclic ADP-ribose; $\left[\mathrm{Ca}^{2+}\right]_{\mathrm{i}}$ - intracellular calcium concentration; CCE - capacitative calcium entry; ETC - electron transport chain; cGMP - cyclic guanosine monophosphate; GSH/GSSG - glutathione (reduced/oxidized); $\mathrm{H}_{2} \mathrm{O}_{2}$ - hydrogen peroxide; $\mathrm{Kv}$ - voltage-gated potassium channel; NADH/NAD ${ }^{+}$- nicotinamide adenine dinucleotide (reduced/oxidized); ROS - reactive oxygen species

Figure 5: Single-lung anesthesia (modified from Ref. Current Opinion in Anaesthesiology. 19(1):34-43, February 2006): a) bronchoscopy revealing endotracheal lesion; b) a double-lumen tube that permits single-lung ventilation; c) two-lung ventilation during exposure of the operative field (top, red arrows highlight lack of oxygenation to a region of the lung), and collapse of the operative lung after inflation of the occluder (bottom, white arrows indicate reduction in blood flow to the collapsed lung) resulting in single-lung ventilation/anesthesia.

Figure 6: Pulmonary vascular remodeling in pulmonary hypertension. Cross-section of a normal pulmonary arteriole and a pulmonary arteriole with pulmonary hypertension. All three layers of the pulmonary vessel undergoes remodeling in pulmonary hypertension, including proliferation of abnormal endothelial cells in the intima, hypertrophy, proliferation and distal migration of smooth muscle cells, proliferation of fibroblasts with increased extracellular matrix deposition, and increased recruitment of leukocytes in the adventitia.

Image adapted and modified from Gordeuk VR, Castro OL, and Machado RF. Pathophysiology and treatment of pulmonary hypertension in sickle cell disease. Blood. 2016;127:820-828. 
Figure 7: Mitochondria exist in fragmented networks in PAH. Mitochondria from 3 adjacent PAH PASMC. The nuclei are not stained in this image and in aggregate the mitochondria in these 3 cells create the artistic impression of a dragon's head. The mitochondria are stained red with the potentiometric dye tetamethyl-rhodamine (TMRM). Scale bar: $20 \mu \mathrm{m}$.

Figure 8: A simplified scheme of mitochondrial fission and fusion in mammalian cells.

(A) Schematic representation of fusion. The outer membrane of two adjacent mitochondria are tethered by the interaction in trans of the HR2 domains of mitofusins (Mfns). GTP binding and hydrolysis cause conformational change of Mfns leading to outer mitochondrial membrane (OMM) fusion. Following OMM fusion, OPA1 drives inner mitochondrial membrane (IMM) fission. (B) Schematic representation of fission. Fission is initiated by endoplasmic reticulum (ER) mediated pre-Drp1 constriction and marks the site for further constriction. Drp1 is recruited from the cytosol to the fission site via its receptors (Mff, MiD49 and MiD51) and forms contractile rings at the fission site. GTP hydrolysis leads to Drp1 conformational changes and constriction. Following this, Dnm2 is recruited to the constricted neck and further constriction (scission) occurs to complete fission. Then the fission machinery is disassembled by ubiquitination and proteosomal degradation [reviewed in(8)].

Figure 9: Mitochondria are fragmented in PAH. Representative images of mitochondrial networks of normal PASMC and PAH PASMC stained with the potentiometric dye TMRM (red). Mitochondrial network appear more fragmented in PAH PASMC as compared to the mitochondria from normal PASMC. 
Reproduced from Circulation 138(3):287-304. Copyright (C) 2019 Circulation

Figure 10: Schematic representations of the proposed role of epigenetically mediated upregulation of MiD49 and MiD51 in PAH

A) Upregulation of MiDs on the outer mitochondrial membrane (OMM) increase mitochondrial fission and promote cell proliferation in pulmonary arterial hypertension (PAH) pulmonary artery smooth muscle cells (PASMC). Downregulated of miR-34a-3p expression accounts for the increased MiD expression and contributes to the pathogenesis of PAH.

B) Silencing of MiD49 and MiD51, by siMiD49 and siMiD51, or by administering miR-34a-3p to PAH PASMC, promotes fusion and attenuates proliferation of PAH PASMC.

Figure 11: Silencing of MiD49 and MiD51 inhibits mitochondrial fission in PAH PASMC.

A) Mitochondrial fragmentation in PAH PASMC is reversed by silencing of MiD49 or MiD51. Representative images of mitochondrial networks of PAH PASMC. PAH PASMC were transfected with the specified siRNA, infected with Adv-mNeon Green and imaged after $48 \mathrm{~h}$ following infection. Mitochondria were color coded by their morphology: green: punctate; red: intermediate; purple: filamentous. Scale bar: $10 \mu \mathrm{m}$.

B) Silencing of MiD49 or MiD51 inhibits mitochondrial fission. Mitochondrial fragmentation was quantified by mitochondrial fragmentation count (MFC) on the left and by a machine learning algorithm that quantified the percentage area of punctate, intermediate and filamentous mitochondria of each image (on the right side of panel B). Adopted from Circulation 138(3):287304). Copyright (C) 2019 Circulation. 
Figure 12: Schematic representation of the proposed mechanism for metabolic reprogramming in pulmonary hypertension fibroblast (PH-Fibs). In PH-Fibs, an alternative splicing complex containing PTBP1 (polypyrimidine tract binding protein 1), hnRNP (heterogeneous nuclear ribonucleoprotein) A1, and hnRNPA2 regulate the state of pyruvate kinase muscle (PKM) isoform expression. In the presence PTBP, exon 10 is included in the mature PKM transcript, whereas exon 9 is excluded, resulting in an increased PKM2/PKM1 ratio which is an important mediator of aerobic glycolysis (increased ) and increased proliferation. Expression of PTBP1 is modulated by its upstream regulator, microRNA-124 [miRNA-124]. miR-124 mimic, siPTBP1, TEPP-46, shikonin (PKM2 inhibitors) and treatment with HDACi restores normal PKM2/PKM1 ratio and reverse Warburg effect in PH-Fib. miR-124-PTBP1-PKM axis is a potential therapeutic target for $\mathrm{PH}$.

Modified from Circulation 2017 Dec 19;136(25):2468-2485.

Figure 13: Role of hypoxia inducible factor-1 $\alpha$ (HIF-1 $\alpha)$ in the PASMC under normoxia, hypoxia and pseudohypoxia (as occurs in PAH)

A) Under normoxic conditions, HIF-1 $\alpha$ is hydroxylated by prolyl hydroxylase domain proteins (PHD), using molecular oxygen, leading to interaction with Von Hipple-Lindau (VHL) and degradation by ubiquitin proteasome pathway.

B) Under hypoxic condition there is a decrease in mitochondrial $\mathrm{H}_{2} \mathrm{O}_{2}$ production and $\mathrm{HIF}-1 \alpha$ expression is stabilized. HIF-1 $\alpha$ translocates to the nucleus where it dimerizes with HIF-1 $\beta$ and recruits co-activators at the hypoxia response element (HRE) to modulate transcription of target genes.

C) In PAH PASMC low SOD2 expression, rather than environmental hypoxia, decreases 
$\mathrm{H}_{2} \mathrm{O}_{2}$ production, creating a pseudohypoxic state, thereby activating HIF-1 $\alpha$. HIF- $1 \alpha$ in turn activates PDK transcription resulting in the inhibition of PDH and further reduction in ROS production. Decreased ROS inhibits certain oxygen- and redox- sensitive potassium channels, including Kv1.5, resulting in PASMC depolarization and calcium overload.

Figure 14: Visualization of mitochondrial DNA replication machinery. Confocal microscopy of a normal human pulmonary artery smooth muscle cell (PASMC) with immunofluorescent labelling of nuclear DNA (blue), mitochondria (red) and transcription factor A mitochondrial (TFAM, green). TFAM is a nuclear-encoded, DNA binding protein that activates transcription of mtDNA; mtDNA replication precedes mitochondrial biogenesis. Scale bar: $5 \mathrm{um}$.

\section{Figure 15: Increased level of miR-25 and miR-138 in PAH-PASMC directly inhibit the expression of mitochondrial calcium uniporter (MCU).}

The loss of MCU expression, exacerbated by increased expression of mitochondrial calcium uptake protein 1 (MICU1), reduces the function of the MCU complex. This simultaneously overloads the cytosolic calcium pool while depriving the mitochondria of calcium. The former triggers pulmonary artery smooth muscle cell migration and proliferation (and vasoconstriction), whereas the latter affects mitochondrial metabolism, inhibiting pyruvate dehydrogenase and promoting a shift to uncoupled glycolysis (the Warburg phenomenon). In aggregate, these epigenetic changes promote cell proliferation and apoptosis resistance. IP3: inositol 1,4,5trisphosphate receptor; VDAC: voltage-dependent anion channel.

Reprinted with permission of the American Thoracic Society. Copyright (C) 2019 American Thoracic Society. 
Figure 16: Time course of metabolic changes on ${ }^{18}$ FDG PET scans of the lung in rats with MCT-PAH

A) Pulmonary arterial acceleration time (PAAT) was measured by pulsed-wave Doppler echocardiography. Measurements were made before MCT injection and weekly thereafter. The arrow at the 3-week time point indicates systolic notching of the pulmonary artery Doppler envelope, typical of severe $\mathrm{PH}$.

B) PAAT is inversely related to the mean pulmonary artery pressure and decreases during the development of pulmonary hypertension. Starting from Week 2, a significant reduction in PAAT is observed.

C) Representative positron emission tomography (PET) scans. Note the increased 18Ffluorodeoxyglucose (FDG) uptake in the right ventricle (RV) and the lung parenchyma of MCT animals. $\mathrm{LV}=$ left ventricle.

D) Quantification of pulmonary ${ }^{18}$ FDG uptake measured with PET. Starting from Week 2, significantly higher lung FDG uptake was observed.

E) Correlation analysis demonstrates the inverse relationship between PAAT and 18FDG uptake.

Seven rats were imaged at each time point.

Reproduced with permission from Marsboom G. et al (2012). "Lung 18F-fluorodeoxyglucose positron emission tomography for diagnosis and monitoring of pulmonary arterial hypertension". American journal of respiratory and critical care medicine 185: 670-679. 
Figure 17: Myocardial perfusion imaging (MPI)-PET (upper Panel), FDG-PET (middle Panel), and FTHA-PET images (lower panel) in three patients with PAH of mild, moderate, or severe degree. The patients' RVEF and mPAP are reported below the images. Note the progressive increase in RV uptake relative to the LV with worsening PAH. Also note that the RV FDG uptake relative to the LV is similar to the RV/LV perfusion tracer uptake in the patients with mild and moderate PAH (left and centre panels), but RV/LV FDG uptake is increased relative to perfusion in the patient with severe PAH (right panel). Thus there is a perfusion/metabolism mismatch in the RV in thsese patients and suggests that there is RV myocardial ischemia or hibernation.

RVEF: Right Ventricular Ejection Fraction, mPAP: Mean Pulmonary Arterial Pressure, RV: Right Ventricle, LV: Left ventricle, PAH: Pulmonary Areterial Hypertension, FDG: ${ }^{18}$ F-fluoro-2deoxyglucose, FTHA: ${ }^{18}$ F-fluoro-6-thioheptadecanoic acid.

Eur Heart J Cardiovasc Imaging. 2016;17:1424-1431

Figure 18: Consequences of pulmonary hypertension include obstructive pulmonary vascular remodeling and right ventricular hypertrophy and dilatation.

Modified from (Can J Cardiol. 2015;31:391-406, Heart. 2006;92 Suppl 1:i2-13).

Figure 19: Active and completed clinical trials returned using search term 'pulmonary hypertension' and filter 'metabolism OR mitochondria'.

Figure 20: Mechanism of right ventricular ischemia in pulmonary hypertension. Right ventricular dysfunction causes increase in right ventricular systolic pressure (RVSP) and right 
ventricular end diastolic pressure (RVENDP) which in turn compresses the left ventricle (LV) leading to the decrease in LV filling, cardiac output and aortic pressure. Decreased aortic pressure and increased RVEDP contribute to decrease in subendocardial blood flow and increase in myocardial oxygen uptake respectively. This finally result in myocardial ischemia. Figure adapted from (Anesthesiology. 2018;128:202-218).

Figure 21: The Randle cycle in the hypertrophied right ventricular cardiomyocyte. The partial inhibition of fatty acid oxidation (FAO), by trimetazidine (TMZ) or ranolazine (RAN), increases pyruvate dehydrogenase (PDH) activity and improves glucose oxidation (GO). The reciprocal relationship between FAO and GO is known as the Randle's cycle. Figure adopted from (J Mol Med (Berl). 2012;90:31-43), with permission.

Figure 22: Dichloroacetate promotes glucose oxidation by inhibiting the pyruvate dehydrogenase kinase (PDK) in right ventricular hypertrophy caused by pulmonary hypertension.

In right ventricular hypertrophy $(\mathrm{RVH})$, activation of various transcription factors, including FOXO1, cMyc and HIF-1 $\alpha$ upregulates expression of many glycolytic gene including pyruvate dehydrogenase kinase (PDK) which is the inhibitor of pyruvate dehydrogenase (PDH) and suppresses mitochondrial respiration. Dichloroacetate (DCA) suppresses glycolysis by inhibiting PDK thereby promoting glucose oxidation. $\mathrm{ETC}=$ electron transport chain, $\mathrm{HK}=$ hexokinase, $\mathrm{H} 2 \mathrm{O} 2$ = hydrogen peroxide, $\mathrm{LDHA}=$ lactate dehydrogrenase A, PFK $=$ phosphofructokinase Figure adopted from Circ Res. 2014;115:176-88 with permission 


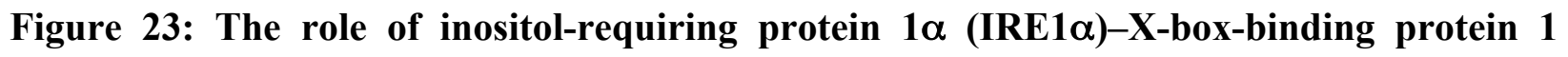
(XBP1s) pathway in heart failure with preserved ejection fraction (HFpEF). High-fat diet (metabolic stress) and hypertension induced by $\mathrm{N}^{\mathrm{w}}$-nitro-L-arginine methyl ester (L-NAME) (mechanical stress) induce symptoms of HFpEF, including impaired filling of left ventricle, reduced exercise capacity, lung congestion, and increased systemic inflammation. Schiattarella et al. noted increased expression of inducible nitric oxide synthase (iNOS), which led to marked overproduction of nitric oxide (NO). Increased NO binds to sulfur atoms of IRE1a, and Snitrosylation decreases IRE1a activity. IRE1a is an important component of the unfolded protein response (UPR), which protects cells from misfolded proteins. Decreased IRE1a activity results in reduced splicing of XBP1s messenger RNA. XBP1s is a transcription factor that activates UPR genes, and the disruption of the UPR is postulated to eventually result in HFpEF. Figure adopted from Amgalan and Kitsis with permission. Nature. 2019;568:324-325, with permission

Figure 24: Schematic diagram of molecular pathways involved in the pathogenesis of PAH. Upstream regulators of mitochondrial mediators, such as microRNA, transcription factors, contribute to the dysregulation of mitochondrial mediator proteins, causing excessive mitochondrial fission/reduced mitochondrial fusion, aerobic glycolysis, increased proliferation and decreased apoptosis, and decreased mitochondrial biogenesis. 


\section{Didactic Figure legend}

Figure 1:

Teaching points: This figure illustrates that homeostatic Oxygen Sensing System (HOSS) is a network of specialized tissues that sense $\mathrm{O} 2$ in their local environments and regulate vascular tone, ventilation or catecholamine secretion to optimize systemic oxygen delivery. The HOSS is made up of: type 1 cells in the carotid body, PASMC, fetoplacental arteries in the placenta, the ductus arteriosus (DA), adrenomedullary chromaffin cells of the adrenal glands, and neuroepithelial bodies, a type of neuroendocrine cell in the airways.

Figure 3A:

Teaching Points: This figure illustrates a hypothesis of oxygen sensing that involves increased ROS production under hypoxia. According to this hypothesis, the Rieske iron-sulfur subunit (RISP) of Complex III is the oxygen-sensor. Furthermore, hypoxia increases ROS and reflects auto-oxidation of the ETC, due to inhibition of the distal ETC. It is the rise in ROS in this model that is proposed to cause HPV.

Figure 3B-E:

Teaching Points: This figure illustrates an earlier and opposing hypothesis of oxygen sensing (compared to Figure 3). In this redox model there is decreased mitochondrial ROS production under hypoxia. NADH dehydrogenase [ubiquinone] iron-sulfur protein 2 (Ndufs2), the quinone binding site in ETC Complex I (and interestingly, not the RISP) has been characterized as the mitochondrial oxygen sensor in both the carotid body and the PASMC. Ndufs2 is the course of mitochondrial ROS and is inhibited by physiologic hypoxia. Inhibition of Ndufs2decreases 
mitochondrial $\mathrm{H}_{2} \mathrm{O}_{2}$ and activates the downstream vasoconstrictor mechanism of HPV. Normal function of Ndufs2 is required for hypoxia-induced increases in cytosolic calcium. Nebulized Ndufs2 reduces expression of Ndufs2 in vivo and inhibits HPV.

Figure 6:

Teaching Points: This figure illustrates the main pathological change of pulmonary vasculature, particularly in small, intrapulmonary arteries and arterioles in PAH. All layers of the pulmonary vessel wall (intima, media, adventitia) are involved in adverse vascular remodelling in PAH. Pathologic changes include intimal hyperplasia, medial hypertrophy, adventitial fibrosis and infiltration of inflammatory cells and progenitor cells.

Figure 8:

Teaching Points: This figure is a simplified schematic representation of mitochondrial fusion and fission. A) The major mediator of mitochondrial fission is dynamin related protein 1 (Drp1), while fusion is mediated by GTPases mitofusin-1 (Mfn1), mitofusin-2 (Mfn2), and optic atrophy 1 (OPA1). During fusion, the outer membrane of two adjacent mitochondria are tethered by the interaction in trans of the HR2 domains of Mfns. This is followed by GTP binding and hydrolysis contributing to the conformational change of Mfn2 which leads to the fusion of OMM. OMM fusion is followed by IMM fusion which is mediated by OPA1. B) During fission, ER-mediated pre-Drp1 constriction marks the site for further constriction. Drp1 is recruited to the mitochondria by its receptors (Mff, MiD49 and MiD51). On the OMM, Drp1 multimerizes forming a contractile

ring at the fission site which is followed by GTP hydrolysis leading to constriction by the conformational changes of Drp1. This is followed by the recruitment of Dnm2 to the constriction 
site and further constriction (scission) occurs to complete fission. Then the fission machinery is disassembled.

Figure 9:

Teaching Points: This figure shows increased mitochondrial fragmentation due to elevated mitochondrial fission in PAH PASMC as compared to normal PASMC. This increase in mitochondrial fission in PAH PASMC is due in part to the increased expression and/or activity of Drp1 and its binding partners, MiD49 and MiD51.

Figure 10:

Teaching Points: This figure represents the role of Drp1 receptors MiD49 and MiD51 in pathogenesis of PAH. In PAH both MiDs are epigenetically upregulated by a decrease in miR34a-3p expression. This increases mitochondrial fission and promotes cell proliferation in PAH PASMC. Silencing of MiD49 and MiD51, by siMiD49 and siMiD51, or by administering miR$34 a-3 p$ to PAH PASMC promotes fusion and attenuates proliferation of PAH PASMC.

Figure 12:

Teaching Points: This figure illustrates the dysregulation of miR-124/PTBP1/PKM pathway in PAH and its targeted treatments. Decreased expression of miR-124 increases the expression of its target, the splicing factor polypyrimidine-tract-binding protein $(P T B P 1)$, resulting in increased PKM2 expression, which enhances uncoupled aerobic glycolysis and increased lactate production while also decreasing translocation of pyruvate to mitochondria. Therefore, miR-124, siPTBP1, or HDACs may be therapeutic agents targeting the miR-124/PTBP1/PKM pathway in PAH. 
Figure 13:

\section{Teaching Points:}

This figure illustrates the regulation of HIF-1 $\alpha$ in normal and pathological conditions (PAH). Under normoxia, the prolyl hydroxylase domain proteins (PHD) and factor inhibiting HIF-1 $\alpha$ (FIH-1) hydroxylates HIF-1 $\alpha$ using molecular oxygen. Hydroxylated HIF-1 $\alpha$ then interacts with Von Hipple-Lindau (VHL) and subsequently degraded by the ubiquitin proteasome pathway. Under hypoxic condition HIF-1 $\alpha$ expression is stabilized and translocates to the nucleus. In the nucleus, it dimerizes with HIF-1 $\beta$ and recruits co-activators at the hypoxia response element (HRE) to initiate transcription of target genes. In PAH, low SOD2 results in decreased $\mathrm{H}_{2} \mathrm{O}_{2}$ creating a pseudohypoxic state activating HIF-1 $\alpha$. This in turn activates PDK resulting in the inhibition of PDH and reduced ROS production. Decreased ROS downregulates Kv1.5 resulting in depolarization and calcium overload.

Figure 15:

\section{Teaching Points:}

This figure illustrates the mechanism of downregulation of MCU in PAH PASMC. Decreased expression of MCU in PAH contributes to increased cytosolic calcium concentration and decreased mitochondrial calcium, which promotes cell proliferation and resistance to apoptosis in PAH PASMC. In PAH two of the upstream regulators of MCU expression are increased, namely miR-25 and miR-138.

Figure 16: 


\section{Teaching Points:}

This figure illustrates a correlation between echocardiography change and glucose uptake in the development of MCT-PAH. The shortening of PAAT is dynamically correlated with increased uptake of FDG in the RV as shown by the PET/CT scanning.

Figure 17:

Teaching Points: This figure indicates a visual assessment to determine the role of RV ischemia and hibernation in patient with different PAH severity. Myocardial perfusion imaging (MPI)-PET (upper panel), ${ }^{18} \mathrm{~F}$-fluoro-2-deoxyglucose (FDG)-PET (middle panel), and ${ }^{18} \mathrm{~F}$-fluoro-6thioheptadecanoic acid (FTHA)-PET images (lower panel) are used to evaluate both glucose and fatty acid metabolism in PAH patients and correlate metabolism with both RV function and the severity of PAH, When the patients were categorized into three groups as mild PAH (mPAP $<35$ $\mathrm{mmHg}$ ), moderate PAH $(35 \leq \mathrm{mPAP}<50 \mathrm{mmHg})$, and severe $\mathrm{PAH}(\mathrm{mPAP} \geq 50 \mathrm{mmHg})$. The patients' RVEF and mPAP are reported below the images.

The ratio of glucose to fatty acid uptake increases as pulmonary artery pressure. The FDG uptake in $\mathrm{RV}$ relative to the $\mathrm{LV}$ is similar to the $\mathrm{RV} / \mathrm{LV}$ perfusion tracer uptake in the patients with mild and moderate PAH (left and centre panels), while in all patients with severe PAH, FDG uptake is higher in the RV relative to the perfusion uptake (a known marker of hibernation) in the patient with severe PAH (right panel). This is a perfusion/metabolism mismatch in the RV and suggests that there is RV myocardial ischemia or hibernation.

Figure 18:

\section{Teaching Points:}


This figure illustrates the main pathological changes in the pulmonary vasculature and right ventricle (RV) in PAH. Pulmonary vascular remodeling includes: plexiform lesions, thrombosis, intimal fibrosis, medial thickening. Right ventricular remodeling has two steps: adaptive RV hypertension (RVH) and maladaptive RVH.

Figure 21:

\section{Teaching Points:}

This figure illustrates the Randle cycle in the right ventricular myocyte. This can be applied in the treatment of PAH. By inhibiting beta-fatty acid oxidation (FAO) using ranolazine, RV function can be improved and glucose oxidation can be increased.

Figure 22:

\section{Teaching Points:}

This figure illustrates the application of inhibiting PDK in the treatment of PAH. Dichloroacetate (DCA) is a small molecular inhibitor of PDK which increases PDH activity thus promoting glucose oxidation. It improves right ventricular hypertrophy caused by pulmonary hypertension.

Figure 24:

\section{Teaching Points:}

This figure illustrates the molecular mechanisms related to mitochondria which contribute to the pathogenesis of PAH. Changes in upstream regulators of mitochondrial mediators contribute to the dysregulation of mitochondrial mediator proteins and result in excessive mitochondrial fragmentation, aerobic glycolysis, increased proliferation and decreased apoptosis, and decreased 
mitochondrial biogenesis. 